Noname manuscript No.

(will be inserted by the editor)

\title{
Differentials on graph complexes III: hairy graphs and deleting a vertex
}

\author{
Marko Živković
}

Received: date / Accepted: date

The author has been partially supported by the Swiss National Science Foundation, grant 200021 150012 and SwissMAP NCCR.

Abstract We continue studying the cohomology of the hairy graph complexes which compute the rational homotopy of embedding spaces, generalizing the Vassiliev invariants of knot theory, after the second part in this series. In that part we have proven that the hairy graph complex $\mathrm{HGC}_{m, n}$ with the extra differential is almost acyclic for even $m$. In this paper we give the expected same result for odd $m$. As in the previous part, our results yield a way to construct many hairy graph cohomology classes by the waterfall mechanism also for odd $m$. However, the techniques are quite different. The main tool used in this paper is a new differential, deleting a vertex in non-hairy Kontsevich's graphs, and a similar map for hairy vertices. We hope that the new differential can have further applications in the study of Kontsevich's graph cohomology. Namely it is conjectured that the Kontsevich's graph complex with deleting a vertex as an extra differential is acyclic.

Keywords Graph complexes · Extra differential · Hairy graph complexes · Deleting a vertex

\section{Introduction}

This paper is the third in the series of papers [5, 6, dealing with extra differentials on graph complexes. In the second paper, 6, the deformed differential was introduced for hairy graphs $\mathrm{HGC}_{m, n}$ for even $m$ and both parities of $n$, and we proved that the complex with that differential is almost acyclic. The main purpose of this paper is to prove the same for odd $m$, as conjectured there, [6, Conjecture 1], finishing the program for hairy graph complexes. A reader familiar to those papers and the importance of their results can skip the first, general part of this introduction, since it mainly repeats what was explained earlier.

\subsection{General introduction}

Generally speaking, graph complexes are graded vector spaces of formal linear combinations of isomorphism classes of some kind of graphs, with the standard differential defined by vertex splitting (or, dually, edge contraction). The various graph cohomology theories are arguably some of the most fascinating objects in homological algebra. Each of graph complexes play a certain role in a subfield of homological algebra, algebraic topology or mathematical physics. They have an elementary and simple combinatorial definition, yet we know almost nothing about what their cohomology actually is.

\footnotetext{
M. Živković

Mathematics Research Unit, University of Luxembourg, Grand Duchy of Luxembourg

2, Avenue de l'Université, L-4365 Esch-sur-Alzette

Tel.: (+352) 4666445248

Fax: $(+352) 46664435248$

E-mail: marko.zivkovic@uni.lu
} 
The most basic graph complexes are introduced by Maxim Kontsevich in [8, 9. These complexes come in versions $\mathrm{GC}_{n}$, where $n$ ranges over integers (see 2.2 for the definition). Physically, $\mathrm{GC}_{n}$ is formed by vacuum Feynman diagrams of a topological field theory in dimension $n$. Alternatively, GC governs the deformation theory of $E_{n}$ operads in algebraic topology [19] or stable cohomology of the algebraic polyvector fields 20]. Some examples of graphs are:

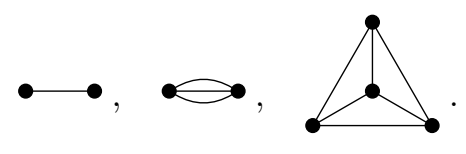

There are many other graph complexes, and we mention only some of them. Ribbon graph complexes describe the cohomology of the moduli spaces of curves $[13,12,8$. Lie decorated graph complexes describe the cohomology of the automorphisms of a free group and play a central role in many results in low dimensional topology [3,8. Oriented graph complexes, that are quasi-isomorphic to Kontsevich's graph complexes [21,11,23, govern for example the quantization of Lie bialgebras [10.

In this paper, apart from Kontsevich's graph complex, we consider hairy graph complexes. These are complexes spanned by graphs with external legs ("hairs"). These complexes come in versions $\mathrm{HGC}_{m, n}$ where $m$ and $n$ range over integers (see 2.4 for the definition). They compute the rational homotopy of the spaces of embeddings of disks modulo immersions, fixed at the boundary $\overline{\operatorname{Emb}_{\partial}}\left(\mathbb{D}^{m}, \mathbb{D}^{n}\right)$, provided that $n-m \geq 3$, see [1,4]. Furthermore, the diagrams enumerating Vassiliev invariants of knot theory appear as the top cohomology of the hairy graph complex $\mathrm{HGC}_{1,3}$. Some examples of hairy graphs are:

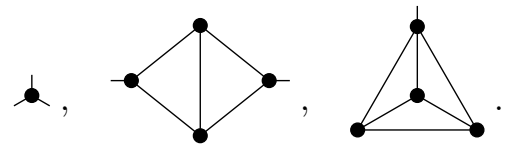

Both kinds of complexes split into the product of subcomplexes with fixed loop order and hairy graph complex splits into the product of subcomplexes with fixed number of hairs, cf. (2.26) and (2.28). Furthermore, the complexes $\mathrm{GC}_{n}$ and $\mathrm{GC}_{n^{\prime}}$, respectively $\mathrm{HGC}_{m, n}$ and $\mathrm{HGC}_{m^{\prime}, n^{\prime}}$, are isomorphic up to some unimportant degree shifts if $m \equiv m^{\prime} \bmod 2$ and $n \equiv n^{\prime} \bmod 2$. Hence it suffices to understand 2 possible cases of $\mathrm{GC}_{n}$ and 4 possible cases of $\mathrm{HGC}_{m, n}$ according to parity of $m$ and $n$.

The long standing open problem we are attacking in this paper is the following.

Open Problem: Compute the cohomologies $\mathcal{H}\left(\mathrm{GC}_{n}\right)$ and $\mathcal{H}\left(\mathrm{HGC}_{m, n}\right)$.

Very little is known about those homologies, and very few tools are available to compute them. The most non-trivial result about Kontsevich's graph cohomology is that zero-th cohomology $\mathcal{H}^{0}\left(\mathrm{GC}_{2}\right)$ is isomorphic to Grothendiech-Teichmüller Lie algebra $\mathfrak{g r t}_{1}$, shown by Willwacher in [19. About the hairy graph complex it is known [18, Propositions 4.1 and 4.4] that the first cohomology of the 2-hair subspace $\mathcal{H}^{-1}\left(\mathrm{H}^{2} \mathrm{HGC}_{1,3}\right)$ and the first cohomology of the 3 -hair subspace $\mathcal{H}^{1}\left(\mathrm{H}^{3} \mathrm{HGC}_{2,3}\right)$ are each isomorphic to the third cohomology of the non-hairy graph complex $\mathcal{H}^{-3}\left(\mathrm{GC}_{3}\right)$. Classes in low degrees have been computed by hand or with computer assistance [7,2, and the Euler characteristics have been computed in 15, 1, 14.

This paper, together with two previous papers from this series [5, 6], deals with extra differentials on graph complexes. The basic idea is to deform the standard differential $\delta$ to $\delta+\delta^{\text {extra }}$ making the complex (almost) acyclic. The extra piece does not fix loop order or the number of hairs, and a spectral sequence can be found such that the standard differential is the first differential. Therefore, on the first page of the spectral sequence we see the standard cohomology we are interested in, and because the whole differential is acyclic, classes of it cancel with each other on further pages, cf. Table 4 . We call this the cancelling mechanism. We can say that classes come in pairs.

In the first paper of the series [5] a deformed differential was introduced for non-hairy graphs $\mathrm{GC}_{n}$ for both parity of $n$. In the second paper of the series [6] a deformed differential was introduced for hairy graphs $\mathrm{HGC}_{m, n}$ for even $m$ and both parities of $n$ :

$$
\Gamma \mapsto \delta \Gamma+\sum_{S}\left(\left.\right|_{\Gamma} ^{\prime} S\right.
$$


summed over all subsets $S$ of the set of hairs with at least two elements. For odd $m$ in [6] the existence of the suitable extra differential $\Delta$ was just conjectured. $\Delta$ transforms a hair into an edge, connecting a hairy vertex with another vertex in all possible ways, see Subsection 2.5 for details.

Conjecture 1 ([6, Conjecture 1]). The complex $\left(\mathrm{HGC}_{-1, n}, \delta+\Delta\right)$ is almost acyclic for all $n$.

We call the spectral sequence arising from differential (1.1), respectively $\delta+\Delta$, the first.

Furthermore, [19], [16] and [17] introduce other deformed differentials on $\mathrm{HGC}_{n, n}$ and $\mathrm{HGC}_{n-1, n}$ making them (almost) quasi-isomorphic to $\mathrm{fGCc}_{n}^{\geq 2}$ :

$$
\mathcal{H}\left(\mathrm{HGC}_{n, n}, D^{\prime}\right) \cong \mathcal{H}\left(\mathrm{fGCc}_{n}^{\geq 2}\right), \quad \mathcal{H}\left(\mathrm{HGC}_{n-1, n}, D^{\prime}\right) \cong \mathcal{H}\left(\mathrm{fGCc}_{n}^{\geq 2}\right),
$$

see Theorem C.1. One checks that all classes that come from $\mathcal{H}\left(\mathrm{GC}_{n}\right)$ live in one-hair part of $\mathcal{H}(\mathrm{HGC})$. Therefore, the spectral sequence argument and the canceling mechanism can be used for this extra differential for other classes of $\mathcal{H}(\mathrm{HGC})$, so they come in pairs. We call the spectral sequence arising from $D^{\prime}$ the second. These pairs are different from those that come from the first spectral sequence.

One can start from one-hair classes that come from $\mathcal{H}(\mathrm{GC})$, find their pairs using the first spectral sequence, then find its pair using the second spectral sequence, and so on. This mechanism is called "waterfall mechanism". The mechanism is introduced in [6] and we describe it roughly in Appendix C.

\subsection{Speciality of this paper}

The main purpose of this paper is to prove Conjecture 1 ([6. Conjecture 1]), making the tentative waterfall mechanism real also in the case of odd $m$.

\section{Theorem 1.1.}

- The complex $\left(\mathrm{HGC}_{-1,1}, \delta+\Delta\right)$ is acyclic.

- The cohomology $\mathcal{H}\left(\mathrm{HGC}_{-1,0}, \delta+\Delta\right)$ is one-dimensional, the class being represented by a graph with one vertex and three hairs on it.

This odd case appears to be much more complicated than even case dealt in [6]. In even case hairs and edges have the same parity, so hairs can be understood as edges, say towards a special vertex. In this picture, the extra peace in the deformed differential [1.1) is nothing but splitting the special vertex. The natural extension of the standard differential is easily seen to be acyclic because of the role of the special vertex.

In the odd case the added differential $\Delta$ is not that nice. Unlike in the even case, extra part $\Delta$ is not of the same kind as standard differential (splitting a vertex), but something else (transforming a hair to an edge). It turns out that just the differential $\Delta$ does not make any simple complex acyclic (see discussions at the beginnings of Sections 6 and 7). Technical complexity of this article is in avoiding those difficulties.

To do that we introduce a new operation called deleting a vertex. In non-hairy Kontsevich's complex $\mathrm{GC}_{n}$ we denote it by $D$ and it deletes a vertex and reconnects its edges to other vertices in all possible ways, summed over all vertices. For $n=-1 D$ is of degree 1 , and under some weak assumptions it is again a differential.

We hope that deleting a vertex $D$ can have further uses. However, in this paper we only get one small extra result in Corollary 3.11 strengthening the result from the first paper in this series about $\mathcal{H}\left(\mathrm{fGC}_{n}\right)$ for even $n$ (cf. [5, Corollary 4]). Computer calculations imply that the complex $\left(\mathrm{GC}_{-1}, D\right)$ is far from acyclic, but there is a hope that $\left(\mathrm{GC}_{-1}, \delta+D\right)$ is acyclic, as stated in the following conjecture. If so, it will lead to a kind of waterfall mechanism in $\mathrm{GC}_{n}$ for odd $n$.

Conjecture 2. Kontsevich's graph complex with the combined differential $\left(\mathrm{GC}_{-1}, \delta+D\right)$ is acyclic.

In the hairy complex we will introduce a sort of deleting a vertex only in certain cases with low number of hairs, needed in this paper. They are probably auxiliary maps with less general importance than $D$.

As shown in Theorem 1.1 $\left(\mathrm{HGC}_{-1, n}, \delta+\Delta\right)$ is acyclic for $n$ odd, while for $n$ even it has one class of cohomology. By the definition, those complexes are spanned by connected graphs. The version that includes disconnected graphs stays acyclic for $n$ odd, but for $n$ even the class in connected version produces a number of non-trivial classes in disconnected version. They are studied in Corollary 7.15. It introduces a list of quasi-isomorphisms from some part of non-hairy graph complexes to the hairy graph complex. 


\section{Structure of the paper}

In Section 2 we define notions, recall results needed in this paper and systematize the notation. Section 3 introduces the deleting a vertex $D$ in non-hairy graphs and provides some results about it. In Section 4 we introduce some new subcomplexes of hairy graph complex needed later in the paper, while Section 5 introduces few maps that delete vertices in hairy complex and shows some needed results. Finally, in sections 6 and 7 we prove the first and the second part of Theorem 1.1. Appendix A clarifies the use of complexes with distinguishable vertices. All technical results are moved to the second Appendix B. The last Appendix C recalls waterfall mechanism and gives some examples for illustration.

Acknowledgements

I am very grateful to Thomas Willwacher for reading the draft of this paper and many suggestions. I also thank Anton Khoroshkin for a fruitful discussion.

\section{Background and definitions}

In this section we recall basic notation and several results shown in the literature that will be used in the paper.

\subsection{General notation}

We work over a field $\mathbb{K}$ of characteristic zero. All vector spaces and differential graded vector spaces are assumed to be $\mathbb{K}$-vector spaces.

Graph complexes as vector spaces are generally defined by the graphs that span them. When we say a graph in a graph complex, we only mean the base graph, while any linear combination (or a series) of graphs will be called an element of the graph complex.

Let $([C], d)$ be a graph complex spanned by a set of graphs $C$ and $[D] \subset[C]$ be a subspace spanned by a set of graphs $D \subset C$. If $[D]$ is closed under the differential, $([D], d)$ is a subcomplex. Let $D^{c}=S \backslash D$ be the complement of $D$ in $C$. If $[D]$ is not closed under the differential, but $\left[D^{c}\right]$ is, we can still safely define the complex $([D], d)$, and assume that we get zero every time when the differential gives a term outside of $[D]$. Technically, that complex is the quotient $([C], d) /\left(\left[D^{c}\right], d\right)$.

\subsection{Kontsevich's graph complexes}

In this subsection we quickly recall the construction of the hairless graph complexes. For more details see [19], or for more elementary definition see [22].

Consider the set of directed graphs $g r a \underset{v}{\geq}, e$ with $v>0$ distinguishable vertices and $e \geq 0$ distinguishable directed edges (numbered from 1 to $v$, respectively $e$ ), all vertices being at least $i$-valent $(i \in\{0,1,2,3\})$, without tadpoles (edges that start and end at the same vertex).

For $n \in \mathbb{Z}$ we define a degree of an element of $g r a \underset{v}{\geq}, e$ to be $d=(v-1) n+(1-n) e$. We may say that the degree of a vertex is $n$ and the degree of an edge is $1-n$. Let

$$
V_{v, e}^{\geq i}:=\left\langle g r a \frac{\geq i}{v, e}\right\rangle[-(v-1) n-(1-n) e]
$$

be the vector space of formal series of $g r a \frac{\geq i}{v}$ with coefficients in $\mathbb{K}$. It is a graded vector space with non-zero term only in degree $d=(v-1) n+(1-n) e$.

Let $S_{k}$ be the $k$-th symmetric group. There is a natural right action of the group $S_{v} \times\left(S_{e} \ltimes S_{2}^{\times e}\right)$ on $g r a \frac{\geq i}{\geq}$, , where $S_{v}$ permutes vertices, $S_{e}$ permutes edges and $S_{2}^{\times e}$ changes the direction of edges. Let $\operatorname{sgn}_{v}, \operatorname{sgn}_{e}$ and $\operatorname{sgn}_{2}$ be one-dimensional representations of $S_{v}$, respectively $S_{e}$, respectively $S_{2}$, where the odd permutation reverses the sign. They can be considered as representations of the whole product $S_{v} \times\left(S_{e} \ltimes S_{2}^{\times e}\right)$. 
The full graph complex is

$$
\mathrm{fGC}_{n}^{\geq i}:= \begin{cases}\prod_{v, e}\left(V_{v, e}^{\geq i} \otimes \operatorname{sgn}_{e}\right)^{S_{v} \times\left(S_{e} \ltimes S_{2}^{\times e}\right)} \simeq \prod_{v, e}\left(V_{v, e}^{\geq i} \otimes \operatorname{sgn}_{e}\right)_{S_{v} \times\left(S_{e} \ltimes S_{2}^{\times e}\right)} & n \text { even, } \\ \prod_{v, e}\left(V_{v, e}^{\geq i} \otimes \operatorname{sgn}_{v} \otimes \operatorname{sgn}_{2}^{\otimes e}\right)^{S_{v} \times\left(S_{e} \ltimes S_{2}^{\times e}\right)} \simeq \prod_{v, e}\left(V_{v, e}^{\geq i} \otimes \operatorname{sgn}_{v} \otimes \operatorname{sgn}_{2}^{\otimes e}\right)_{S_{v} \times\left(S_{e} \ltimes S_{2}^{\times e}\right)} n \text { odd. }\end{cases}
$$

Here the group in the subscript means taking the space of coinvariants, and the group in superscript means taking the space of invariants. Because the group is finite, the two spaces are isomorphic to each other, as stated.

If there is a multiple edge in a graph (more than one edge between the same par of vertices), switching them does not change the graph. For $n$ even switching edges changes the sign in definition (2.2), so graphs with a multiple edge are zero in space of coinvariants $\mathrm{fGC}_{n}^{\geq i}$. Therefore, for even $n$ me may assume there are no graphs with multiple edges.

Note that the full graph complex is actually a graded space, called complex by abuse of notation, as many others in this paper. It becomes a complex after adding a differential. The standard differential $\delta$ on $\mathrm{fGC}_{n}$ is defined as follows:

$$
\delta(\Gamma):=\sum_{x \in V(\Gamma)} \frac{1}{2} s_{x}(\Gamma)-a_{x}(\Gamma),
$$

where $V(\Gamma)$ is the set of vertices of $\Gamma, s_{x}$ stands for "splitting of $x$ " and means inserting $\bullet$ instead of the vertex $x$ and summing over all possible ways of connecting the edges that have been connected to $x$ to the new two vertices, and $a_{x}$ stands for "adding an edge at $x$ " and means adding ${ }^{x} \bullet \bullet$ on the vertex $x$. Unless $x$ is an isolated vertex, $a_{x}$ will cancel two terms of the splitting $s_{x}$. To precisely define the sign of the resulting graph, we set that, before acting of $S_{v} \times\left(S_{e} \ltimes S_{2}^{e}\right)$, a new vertex and an edge get the next free number and the edge is directed towards the new vertex. If a vertex next to an $N$-fold edge ( $N$ edges between same two vertices) is split such that one new vertex gets $k$ edges, and another $N-k$, there is a factor $\left(\begin{array}{l}N \\ k\end{array}\right)$, coming from distinguishing edges. One can check that the minimal valence condition $\geq i$ for $i \in\{0,1,2,3\}$ is preserved under the differential, so the complex is well defined. It is also clear that the differential is indeed of degree 1 .

Since the differential does not change the number $e-v$, the full graph complex $\mathrm{fGC}_{n}^{\geq i}$ splits into the direct product of subcomplexes:

$$
\left(\mathrm{fGC}_{n}^{\geq i}, \delta\right)=\left(\prod_{b \in \mathbb{Z}} \mathrm{B}^{b} \mathrm{fGC}_{n}^{\geq i}, \delta\right)
$$

where $\mathrm{B}^{b} \mathrm{fGC}_{n}^{\geq i}$ is the part of $\mathrm{fGC}_{n}^{\geq i}$ spanned by graphs in which $e-v=b$.

For the chosen parity of $n$, the actual number $n$ matters only for the degree shift of the subcomplexes $\mathrm{B}^{b} \mathrm{fGC}_{n}^{\geq i}$ in the product. If we know the cohomology for one $n$, cohomology for another $n$ of the same parity is straightforward. Therefore, in this paper we will only deal with the complexes $\mathrm{fGC}_{0}^{\geq i}$ and $\mathrm{fGC}_{1}^{\geq i}$, as representatives for the even and the odd complexes. Note that in $\mathrm{fGC}_{0}^{\geq i}$ the degree is $d=e$ and in $\mathrm{fGC}_{1}^{\geq i}$ the degree is $d=v-1$.

Let

$$
\mathrm{fGCc}_{n}^{\geq i} \subset \mathrm{fGC}_{n}^{\geq i}
$$

be the subcomplex spanned by the connected graphs.

If $i=0$ we will usually omit the superscript $\geq 0$, e.g. we consider

$$
\mathrm{fGC}_{n}:=\mathrm{fGC}_{n}^{\geq 0} \text {. }
$$

We introduce a shorter notation

$$
\mathrm{GC}_{n}:=\mathrm{fGCc}_{n}^{\geq 3}
$$

because that complex is particularly important.

Cohomology of these subcomplexes is related to the cohomology of the full complexes, as shows the following result. 
Proposition 2.1 ([19, Proposition 3.4]). The cohomologies satisfy

$$
\begin{aligned}
& \mathcal{H}\left(\mathrm{fGCc}_{0}\right)=\mathcal{H}\left(\mathrm{fGCc}_{0}^{\geq 2}\right)=\mathcal{H}\left(\mathrm{GC}_{0}\right) \oplus \bigoplus_{j \geq 1} \mathbb{K}[-4 j-1], \\
& \mathcal{H}\left(\mathrm{fGCc}_{1}\right)=\mathcal{H}\left(\mathrm{fGCc}_{1}^{\geq 2}\right)=\mathcal{H}\left(\mathrm{GC}_{1}\right) \oplus \bigoplus_{j \geq 0} \mathbb{K}[-4 j-2] .
\end{aligned}
$$

\subsection{The spectral sequence of [5]}

In the first paper of this series we introduced deformed differentials on the graph complexes above. In this paper we only need the even case, $n=0$. There is an extra differential $\nabla$ on $\mathrm{fGC}_{0}$ that acts by adding one edge in all possible ways, see Figure 1. Every edge is added twice, once from one vertex to the other and once in the other way round.

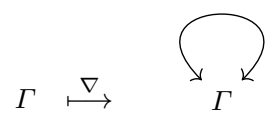

Fig. 1 The action of $\nabla$ to the graph $\Gamma$. The graph at the right means the sum over all graphs that can be formed by attaching arrow ends to vertices, without making a tadpole.

It holds that $\delta \nabla+\nabla \delta=0$, so the differential on $\mathrm{fGC}_{0}$ can be deformed to $\delta+\nabla$. The result we need is the following.

Proposition 2.2 ([5, Corollary 4]). There is a spectral sequence converging to

$$
\mathcal{H}\left(\mathrm{fGCc}_{0}, \delta+\nabla\right)=0
$$

whose $E^{1}$ term is

$$
\mathcal{H}\left(\mathrm{fGCc}_{0}, \delta\right) .
$$

The result implies that the homological classes of $\mathcal{H}\left(\mathrm{fGCc}_{0}, \delta\right)$ have to be canceled on later pages of the spectral sequence. Therefore they come in pairs.

\subsection{Hairy graph complex}

The hairy graph complexes $\mathrm{HGC}_{m, n}$ in general are defined and studied in the second paper of this series, 6]. There are essentially four types of complexes depending on the parity of $n$ and $m$. In this paper we are interested in two types, those with parity of edges and hairs being the opposite, i.e. $m$ is odd. We will deal with the complexes $\mathrm{HGC}_{-1,0}$ and $\mathrm{HGC}_{-1,1}$ as representatives of those types.

For $h \in \mathbb{N}_{0}$ let $\mathrm{H}^{h} \mathrm{fHGC}_{-1, n}^{\geq i}$ be the complex spanned by graphs similar to those of $\mathrm{fGC}_{\bar{n}}^{\geq i}$, but with $h$ hairs attached to vertices, strictly defined as follows. Consider the set of directed graphs $g r a a_{v, e, h}^{\geq i}$ with $v>0$ distinguishable vertices, $e \geq 0$ distinguishable directed edges and $h \geq 0$ distinguishable hairs attached to some vertices, all vertices being at least $i$-valent $(i \in\{0,1,2,3\})$, without tadpoles (edges that start and end at the same vertex). In hairy graphs, the valence includes also the attached hairs, i.e. the valence of a vertex is the number of edges and hairs attached to it.

For $n, m \in \mathbb{Z}$ let a degree of an element of $g r a_{v, e, h}^{\geq i}$ be $d=-m+v n+(1-n) e+(m+1-n) h$. In this paper we consider only the case $m=-1$, so $d=1+v n+(1-n) e-n h$. Let

$$
V_{v, e, h}^{\geq i}:=\left\langle g r a \frac{\geq i}{\geq i, h, h}\right\rangle[-1-v n-(1-n) e+n h]
$$

be the vector space of formal series of $g r a \frac{v i, e, h}{\geq i}$ with coefficients in $\mathbb{K}$. It is a graded vector space with non-zero term only in degree $d=1+v n+(1-n) e-n h$. 
There is a natural right action of the group $S_{v} \times S_{h} \times\left(S_{e} \ltimes S_{2}^{\times e}\right)$ on $g r a \frac{v, e, h}{\geq i}$, where $S_{v}$ permutes vertices, $S_{h}$ permutes hairs, $S_{e}$ permutes edges and $S_{2}^{\times e}$ changes the direction of edges. Let $\operatorname{sgn}_{v}, \operatorname{sgn}_{h}$, $\operatorname{sgn}_{e}$ and $\operatorname{sgn}_{2}$ be one-dimensional representations of $S_{v}$, respectively $S_{h}$, respectively $S_{e}$, respectively $S_{2}$, where the odd permutation reverses the sign. They can be considered as representations of the whole product $S_{v} \times S_{h} \times\left(S_{e} \ltimes S_{2}^{\times e}\right)$.

The full hairy graph complex is

$$
\mathrm{H}^{h} \mathrm{fHGC}_{-1, n}^{\geq i}:= \begin{cases}\prod_{v, e}\left(V_{v, e, h}^{\geq i} \otimes \operatorname{sgn}_{e}\right)^{S_{v} \times S_{h} \times\left(S_{e} \ltimes S_{2}^{\times e}\right)} & \text { for } n \text { even, } \\ \prod_{v, e}\left(V_{v, e, h}^{\geq i} \otimes \operatorname{sgn}_{v} \otimes \operatorname{sgn}_{h} \otimes \operatorname{sgn}_{2}^{\otimes e}\right)^{S_{v} \times S_{h} \times\left(S_{e} \ltimes S_{2}^{\times e}\right)} & \text { for } n \text { odd. }\end{cases}
$$

Because the group is finite, the space of invariants may be replaced by the space of coinvariants.

As in the non-hairy case, for symmetry reasons there are no graphs with multiple edges for even $n$. Similarly, for odd $n$ there are no graphs with multiple hairs on the same vertex.

The standard differential is similar to the one of $\mathrm{fGC}_{n}^{\geq i}$ :

$$
\delta(\Gamma):=\sum_{x \in V(\Gamma)} \frac{1}{2} s_{x}(\Gamma)-a_{x}(\Gamma)-h(x) e_{x}(\Gamma),
$$

where in "splitting of $x " s_{x}$ hairs are also attached in all possible ways to the new two vertices. There is a factor $\left(\begin{array}{l}N \\ k\end{array}\right)$ for splitting a vertex with $N$ hairs into vertices with $k$ and $N-k$ hairs, like in splitting of a multiple edge. "Adding an edge at $x " a_{x}$ is the same as before and $e_{x}$ stands for "extracting a hair at $x$ " and means adding ${ }^{x} \bullet \bullet$ on the vertex $x$ instead of one hair, while $h(x)$ is the number of hairs on the vertex $x$. Unless $x$ is an isolated vertex with a hair, $h(x) e_{x}$ will cancel two terms of the splitting $s_{x}$. To precisely define the sign of the resulting graph, we set that, before acting of $S_{v} \times\left(S_{e} \ltimes S_{2}^{e}\right)$, a new vertex and an edge get the next free number and the edge is directed towards the new vertex. It is easily seen that $\delta$ is indeed a differential, i.e. $\delta^{2}=0$.

Example 2.3. To clarify the signs and cancellations, let us calculate the differential of

$$
\Gamma=\bullet \bullet \cdots \longrightarrow \in \mathrm{H}^{1} \mathrm{fHGC}_{-1, n}^{\geq 0}
$$

with $v$ vertices and $v-1$ edges. Let us chose the representative of the class $\Gamma$ in $V_{v, v-1,1}^{\geq 0}$ to be

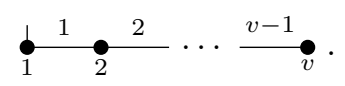

After splitting the last vertex $v s_{v}(\Gamma)$ has two addends, one where the edge $v-1$ is connected to $v$ and one where it is connected to the new vertex $v+1$. One addend can be transformed to another by switching vertices $v$ and $v+1$, and changing the direction of the edge $v$ between them. Both operations change the sign of the class $\Gamma$ if $n$ is odd, so there is never a change at the end and both addends form the same class as $a_{v}(\Gamma)$. Since we have the sum $\frac{1}{2} s_{x}+a_{x}$, those addends cancel with adding an antenna $a_{v}(\Gamma)$.

After splitting a 2-valent vertex $x$ (any vertex other than $v$ ) $s_{x}(\Gamma)$ has two addends with both edges (or the hair) connected to the same vertex, $x$ or $v+1$. It is again seen that both addends form the same class as $a_{x}(\Gamma)$ and they cancel with $a_{x}(\Gamma)$.

The remaining addends of $s_{x}(\Gamma)$ have edges (or the hair) connected to different vertices. It is seen that both addends form the same class, and the class looks like

with $v+1$ vertices. Because of the factor $\frac{1}{2}$, two addends add up to one class.

Let us check if the results of splitting different vertices add up or cancel. For our chosen representative $s_{x}(\Gamma)$ gives

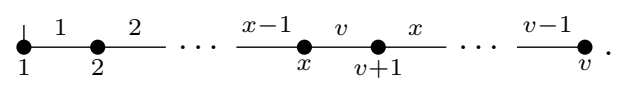


To transform it to the result of splitting the neighbouring vertex $x+1$ we need to switch vertices $v+1$ and $x+1$, and switch edges $v$ and $x$. For any parity of $n$ one switching changes the sign of the class, so the terms cancel.

Extending the hair $h_{1}(\Gamma)$ will end up in the same class. For our chosen representative $h_{1}$ gives

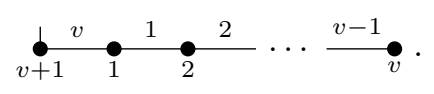

One can see that this cancels with splitting the first vertex $s_{1}(\Gamma)$.

In the result we have addends $h_{1}(\Gamma), s_{1}(\Gamma), \ldots, s_{v-1}(\Gamma)$ that are all the same up to the sign, and neighbouring addends cancel. Therefore, if $v$ is even the result is $\delta(\Gamma)=0$, and if $v$ is odd it holds that

$$
\delta(\Gamma)=\bullet \bullet \cdots \rightarrow
$$

with $v+1$ vertices.

In particular

$$
\mathrm{H}^{0} \mathrm{fHGC}_{-1, n}^{\geq i}=\mathrm{fGC}_{n}^{\geq i}[-1-n] .
$$

We define the full hairy graph complex

$$
\mathrm{fHGC}_{-1, n}^{\geq i}:=\prod_{h \geq 0} \mathrm{H}^{h} \mathrm{fHGC}_{-1, n}^{\geq i} .
$$

We often need a subcomplex without hairless part:

$$
\mathrm{H}^{\geq 1} \mathrm{fHGC}_{-1, n}^{\geq i}:=\prod_{h \geq 1} \mathrm{H}^{h} \mathrm{fHGC}_{-1, n}^{\geq i} .
$$

The differential does not change the number $e-v$ again, so the full hairy graph complex fHGC $\mathrm{f}_{-1, n}^{\geq i}$ splits into the direct product of (in each degree) finite dimensional subcomplexes:

$$
\left(\mathrm{fHGC}_{-1, n}^{\geq i}, \delta\right)=\left(\prod_{b \in \mathbb{Z}} \mathrm{B}^{b} \mathrm{fHGC}_{-1, n}^{\geq i}, \delta\right),
$$

where $\mathrm{B}^{b} \mathrm{fHGC}_{-1, n}^{\geq i}$ is the part of $\mathrm{fHGC}_{n}^{\geq i}$ spanned by graphs where $e-v=b$. The same is true for $\mathrm{H}^{\geq 1} \mathrm{fHGC}_{-1, n}^{\geq i}$.

Similarly to the non-hairy complex, for a chosen parity of $n$, the actual number $n$ matters only for the degree shift of the subcomplexes $\mathrm{B}^{b} \mathrm{fHGC}_{-1, n}^{\geq i}$ in the product. If we know the cohomology for one $n$, cohomology for another $n$ of the same parity is straightforward. Therefore, in this paper we will only deal with complexes $\mathrm{HHGC}_{-1,0}^{\geq i}$ and $\mathrm{fHGC}_{-1,1}^{\geq i}$, and their subcomplexes, as representatives for even and odd complexes. Note that in $\mathrm{fHGC}_{-1,0}^{\geq i}$ the degree is $d=e+1$ and in $\mathrm{fHGC}_{-1,1}^{\geq i}$ the degree is $d=v+1-h$.

Similarly to the non-hairy complex, let

$$
\mathrm{fHGCc}_{-1, n}^{\geq i} \subset \mathrm{fHGC}_{-1, n}^{\geq i}
$$

be the subcomplex spanned by connected graphs.

In the hairy case, we skip the superscript if $i=1$, e.g. we consider

$$
\text { fHGC }_{-1, n}=\text { fHGC }_{-1, n}^{\geq 1},
$$

because those complexes will be used the most, unlike in the non-hairy case where skipping superscript means including all valences. We also introduce a shorter notation

$$
\mathrm{HGC}_{-1, n}:=\mathrm{H}^{\geq 1} \mathrm{fHGCc}_{-1, n}^{\geq 3} \text {. }
$$

This complex is the closest to the one defined in [6]. Strictly speaking, $\mathrm{HGC}_{-1, n}$ for odd $n$ defined in 6] has another graph that we do not allow here, the graph with no vertices and two hairs. It comes naturally if a hair is understood as an edge towards 1-valent vertex of another type, being the case in that paper. 
In [6] an extra differential $\Delta$ has been defined on $\mathrm{HGC}_{-1, n}$ that anti-commutes with $\delta$, so that $\delta+\Delta$ is also a differential. Here, on $\mathrm{fHGC}_{-1, n}^{\geq i}$ we define the following additional operation $\Delta: \mathrm{H}^{h} \mathrm{fHGC}_{-1, n}^{\geq i} \rightarrow$ $\mathrm{H}^{h-1} \mathrm{fHGC}_{-1, n}^{\geq i}$ :

$$
\Delta \Gamma=\sum_{x \in V(\Gamma)} \Delta_{x}=\sum_{x \in V(\Gamma)} h(x) \tilde{\Delta}_{x}
$$

where $\tilde{\Delta}_{x}$ deletes a hair on vertex $x$ and connects $x$ to other vertices in all possible ways with a new edge, and $\Delta_{x}=h(x) \tilde{\Delta}_{x}$ for $h(x)$ being the number of hairs on $x$, see example in Figure 2 , To precisely define the sign of the resulting graph, we set that, before the acting of $S_{v} \times S_{h} \times\left(S_{e} \ltimes S_{2}^{\times e}\right)$, the last hair is being deleted and the new edge get the next free number. Quotiented to $\mathrm{HGC}_{-1, n}, \Delta$ is the same differential as defined in [6].

Lemma 2.4. $\Delta$ squares to zero and anti-commutes with $\delta$, so $\delta+\Delta$ is also a differential.

Proof. A straightforward verification, c.f. [6, Lemma 1].

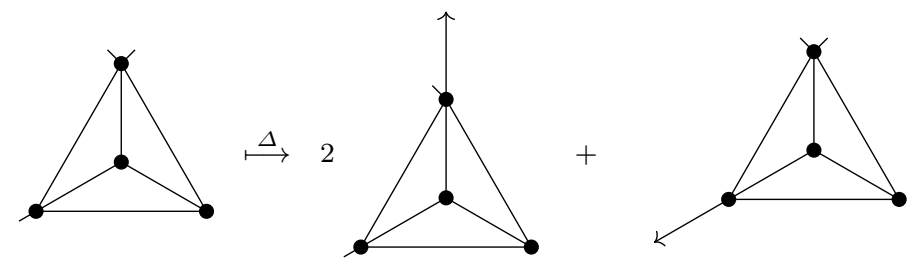

Fig. 2 Example of the action $\Delta$. The graphs at the right mean the sum over all graphs that can be formed by attaching ends of the arrow to vertices, without making a tadpole.

2.6 Adding a hair

We will need the following map. Let

$$
\chi^{1}: \mathrm{H}^{h} \mathrm{fHGC}_{-1, n} \rightarrow \mathrm{H}^{h+1} \mathrm{fHGC}_{-1, n}
$$

be the map that adds a hair in all possible ways. Also, let for even $n$

$$
\begin{gathered}
\chi^{d}: \mathrm{H}^{h} \mathrm{fHGC}_{-1, n} \rightarrow \mathrm{H}^{h+d} \mathrm{fHGC}_{-1, n}, \\
\chi^{d}=\left(\chi^{1}\right)^{d}
\end{gathered}
$$

for $d \geq 2$. It adds $d$ hairs in all possible ways, but there is a multinomial coefficient $\left(\begin{array}{c}d \\ k_{1}, k_{2}, \ldots\end{array}\right)$ before, where $k_{i}$ is the number of hairs added to the vertex $i$. We also set $\chi^{0}=\operatorname{Id}$ and $\chi^{d}=0$ for $d<0$. Check that for odd $n$ the latter map would not make sense because for symmetry reasons adding two hairs in all possible ways gives zero.

Lemma 2.5. For every $d \geq 0$ and every $\Gamma \in \mathrm{H}^{0} \mathrm{fHGC}_{-1,0}$

$$
\Delta \chi^{d}(\Gamma)=d \chi^{d-1} \nabla(\Gamma) .
$$

Proof. Straightforward. 
2.7 Transforming a hair into an antenna

We define two maps

$$
\begin{gathered}
c, c^{(2)}: \mathrm{H}^{h} \mathrm{fHGC}_{-1, n} \rightarrow \mathrm{H}^{h-1} \mathrm{fHGC}_{-1, n}, \\
c(\Gamma)=\sum_{i \in H(\Gamma)} c_{i}(\Gamma) \\
c^{(2)}(\Gamma)=\sum_{i \in H(\Gamma)} c_{i}^{(2)}(\Gamma)
\end{gathered}
$$

where $H(\Gamma)$ is the set of hairs of $\Gamma$ and $c_{i}(\Gamma)$ deletes the hair $i$ at vertex $x$ and adds an antenna like $a_{x}$. Similarly, $c_{i}^{(2)}(\Gamma)$ deletes the hair and adds an antenna of length 2: $\bullet \bullet$. An example is sketched in Figure 3
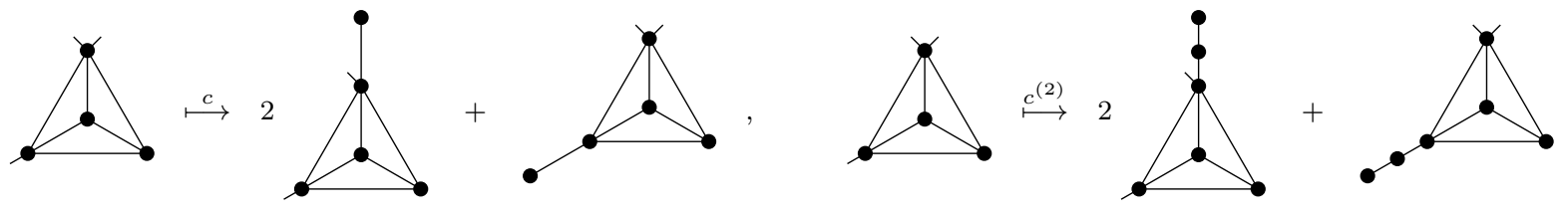

Fig. 3 Examples of the actions $c$ and $c^{(2)}$, transforming a hair into an antenna.

Lemma 2.6. For every $\Gamma \in \mathrm{fHGC}_{-1, n}$ it holds that

$$
\delta c(\Gamma)=c \delta(\Gamma)+c^{(2)}(\Gamma) .
$$

Proof. In $c \delta(\Gamma)$ we first split a vertex, summed over all vertices, and then transform a hair into an antenna, summed over all hairs. After choosing a vertex and a hair we can do the opposite, first transform the hair, and then split the vertex. All addends are the same, and hence commute, except $-h(x) e_{x}$ from $\delta$ (2.10), if the chosen hair is on the chosen vertex $x$. That means, if we first split, there is an addend $-c_{i} e_{x}(\Gamma)=-c_{i}^{(2)}(\Gamma)$ not cancelled by anything in the opposite action.

Moreover, apart from actions considered, $\delta c(\Gamma)$ has an extra term, splitting the new vertex created by $\Gamma$. But that term is 0 because $s_{x}$ and $a_{x}$ cancel. In total, summed over all hairs we have

$$
c \delta(\Gamma)=\delta c(\Gamma)-c^{(2)}(\Gamma)
$$

what was to be demonstrated.

Lemma 2.7. For every $\Gamma \in \mathrm{fHGC}_{-1, n}$ it holds that

$$
\Delta c(\Gamma)=c \Delta(\Gamma)
$$

Proof. Both $c$ and $\Delta$ choose a hair, one is transformed to an antenna and one connected to other vertices. We can do it in any order. Only term that appears in $\Delta c(\Gamma)$ but not in $c \Delta(\Gamma)$ is when $\Delta$ connects a hair $j$ to the new vertex created by $c_{i}$. But that term is cancelled with the term when $\Delta$ connects a hair $i$ to the new vertex created by $c_{j}$, because exchanging chosen hairs for odd $n$, respectively created edges for even $n$, gives the sign -1 . 


\subsection{Some simple graphs}

Here we define some simple graphs. They live in the hairy graph complexes, and if they have no hairs, also in the standard graph complexes.

Let

$$
\sigma_{a}:=
$$

be a graph with one vertex and $a \geq 0$ hairs, and we call it a star. Let

$$
\lambda_{a}:=\bullet
$$

with $a-1$ hairs on one vertex for $a \geq 1$. Hairless graphs that exist in $\mathrm{fGC}_{n}$ are denoted by simpler notation $\sigma:=\sigma_{0}$ and $\lambda:=\lambda_{1}$. Because of symmetry reasons in fHGC $_{-1,1}$ there can not be more than one hair on the same vertex, so in that complex only $\sigma_{1}$ and $\lambda_{2}$ exist, together with hairless $\sigma_{0}$ and $\lambda_{1}$.

For two graphs $\Gamma$ and $\Gamma^{\prime}$, the graph $\Gamma \cup \Gamma^{\prime}$ is the disconnected graph that consists of $\Gamma$ and $\Gamma^{\prime}$. For the matter of sign, all vertices, edges and hairs of the first graph come before those of the second one. For a graph $\Gamma$ and $n \geq 1, \Gamma^{\cup n}$ is the graph that consist of $n$ copies of the graphs $\Gamma$ put together.

The following graph in $\mathrm{HGC}_{-1, n}$ will be used often:

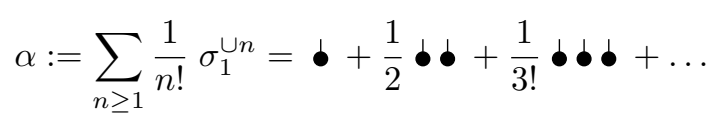

\subsection{List of graph complexes}

For the reader's convenience, we provide Table 1 of all graph complexes used and defined in the paper, with a short explanation and the reference to the definition. Similarly, in Table 2 we provide all differentials and important maps.

The most notations of graph complexes, except some technical complexes, are of the form $\mathrm{pNe}_{\mathrm{i}}^{\mathrm{s}}$, meaning as follows.

- The first small letter p is either f, meaning full complex, either omitted. General complexes are full, but some its subcomplexes are particularly important, and they are labeled without $f$.

- Capital letters $\mathrm{N}$ is the main type of complex. It can be:

- GC - graph complex,

- HGC - hairy graph complex.

- The ending e deals with connectivity and if stated means:

- c - connected graphs,

$-\mathrm{d}$ - disconnected graphs.

- The subscript i defines degrees and parity (1 number for ordinary complexes and 2 numbers for hairy complexes).

- The superscript $\mathrm{s}$ deals mostly with valences and if stated means:

$-\geq i$ - all vertices are at least $i$-valent,

- $\dagger$ - all vertices at least 1 -valent and no connected component $\lambda_{1}$,

- $\ddagger$ - all vertices at least 1 -valent and no connected component $\lambda_{1}, \sigma_{1}$ or $\lambda_{2}$,

- $\bullet$ - all vertices at least 2 -valent and hairy vertices at least 3 -valent.

\subsection{Splitting of complexes and spectral sequences}

We often need subcomplex of the particular complex spanned by graphs that have some number fixed, e.g. number of vertices. Let $v$ be the number of vertices in a graph, $e$ the number of edges, $h$ the number of hairs, $c$ the number of connected components and $p$ the number of tree-like connected components (connected components with 1 vertex more than edges). Prefixes are listed in Table 3 .

We have already used prefixes $\mathrm{B}$ and $\mathrm{H}$ in (2.4), 2.9) and (2.14).

Let $\Gamma$ be an element of a graph complex C. We also use prefixes from above in front of the graph $\Gamma$, indicating the part of $\Gamma$ with fixed number. E.g. $\mathrm{H}^{5} \Gamma$ is the part of $\Gamma$ with 5 hairs. 


\begin{tabular}{|c|c|c|}
\hline Notation & Explanation & Reference \\
\hline $\mathrm{fGC}_{\bar{n}}^{\geq i}$ & Graph complex with graphs whose vertices are at least $i$-valent & 2.2 \\
\hline $\mathrm{fGC}_{n}$ & $=\mathrm{fGC}_{\bar{n}}^{\geq 0}$ & $(2.6)$ \\
\hline $\mathrm{fHGC}_{-1, n}^{\geq i}$ & Hairy graph complex with graphs whose vertices are at least $i$-valent & 2.12 \\
\hline fHGC $_{-1, n}$ & $=\mathrm{fHGC}_{-1, n}^{\geq 1}$ & 2.16 \\
\hline $\mathrm{pNc}_{\mathrm{i}}^{\mathrm{s}}$ & Subcomplex of $\mathrm{pN}_{\mathrm{i}}^{\mathrm{s}}$ spanned by connected graphs & $2.5,, 2.15$ \\
\hline $\mathrm{GC}_{n}$ & $=\mathrm{fGCc}_{n}^{\geq 3}$ & 2.7 \\
\hline $\mathrm{H} \geq 1 \mathrm{pHGCe} \mathrm{i}_{\mathrm{s}}^{\mathrm{s}}$ & $\subset$ pHGCe $_{i}^{s}$ without hairless graphs & 2.9 \\
\hline $\mathrm{HGC}_{-1, n}$ & $=\mathrm{H}^{\geq 1} \mathrm{fGCc}_{n}^{\geq 3}$ & 2.17 \\
\hline $\mathrm{fHGC}^{\dagger}-1, n$ & $\subset$ fHGC $_{-1, n}$ with no connected component $\lambda_{1}$ & Definition 4.2 \\
\hline $\mathrm{fGC}_{n}^{\dagger}$ & $=\mathrm{H}^{0} \mathrm{fHGC}_{-1, n}^{\dagger}[-1-n]$ & Definition 4.2 \\
\hline $\mathrm{fHGC}^{\ddagger}-1, n$ & $\subset$ fHGC $_{-1, n}$ with no connected component $\sigma_{0}, \sigma_{1}$ or $\lambda_{2}$ & Definition $\overline{\overline{4.4}}$ \\
\hline $\mathrm{fHGC}^{\natural}-1, n$ & $\subset$ fHGC ${ }^{2}$ spanned by graphs that do not have 2 -valent vertex with a hair & Definition $\overline{4.6}$ \\
\hline $\mathrm{fHGC}_{-1, n}^{\dagger / \ddagger}$ & $\mathrm{fHGC}_{-1, n}^{\dagger} / \mathrm{fHGC}_{-1, n}^{\ddagger}$ & Definition 4.12 \\
\hline $\mathrm{UR}_{-1, n}$ & $\subset$ fHGC $_{-1, n}^{\dagger}$ with all connected components $\sigma_{1}$ or $\lambda_{2}$ & Definition 4.12 \\
\hline $\mathrm{HL}_{1}$ & $=\left(\Delta+D^{(1)}\right)\left(\mathrm{H}^{1} \mathrm{fHGC}_{-1,1}^{\dagger}\right)$ & 6.6 \\
\hline $\mathrm{HL}_{0}$ & $=\Delta\left(\mathrm{H}^{1} \mathrm{fHGC}_{-1,0}\right)$ & 7.7 \\
\hline $\mathrm{H}^{\mathrm{b}} \mathrm{pHGC}_{-1, n}^{\mathrm{s}}$ & $=\mathrm{H}^{\geq 1} \mathrm{pHGC}_{-1, n}^{\mathrm{s}} \oplus \mathrm{HL}_{n}$ & $\begin{array}{l}6.5),(6.8),(6.9) \\
7.6),(7.8)\end{array}$ \\
\hline fHGCd $_{-1,0}$ & $\subset$ fHGC $_{-1,0}$ spanned by disconnected graphs & $(7.14)$ \\
\hline
\end{tabular}

Table 1 The table of graph complexes used in the paper. Universal letters p, N, e, i and s mean any letter, and also without the letter.

\begin{tabular}{|c|c|l|r|}
\hline Notation & Largest domain and codomain & Explanation & Reference \\
\hline$\delta$ & $\begin{array}{c}\mathrm{fGC}_{n} \rightarrow \mathrm{fGC}_{n} \\
\mathrm{fHGC}_{-1, n}^{\geq 0} \rightarrow \mathrm{fHGC}_{-1, n}^{\geq 0}\end{array}$ & standard differential, splitting a vertex & 2.3 \\
\hline$\nabla$ & $\mathrm{fGC}_{0} \rightarrow \mathrm{fGC}_{0}$ & adding an edge in all possible ways & Figure \\
\hline$\Delta$ & fHGC $_{-1, n}^{\geq 0} \rightarrow \mathrm{fHGC}_{-1, n}^{\geq 0}$ & transforming a hair into an edge & 2.18 \\
\hline$D$ & $\begin{array}{l}\mathrm{fGC}_{0} \rightarrow \mathrm{fGC}_{0} \\
\mathrm{fGC}_{1} \rightarrow \mathrm{fGC}_{1}\end{array}$ & deleting a vertex & 3.1 \\
\hline$D^{(1)}$ & $\mathrm{H}^{1}$ fHGC $_{-1, n} \rightarrow \mathrm{H}^{0} \mathrm{fHGC}_{-1, n}$ & deleting the hairy vertex & 5.1 \\
\hline$D^{(p u s h)}$ & $\mathrm{H}^{1} \mathrm{fHGC}_{-1, n} \rightarrow \mathrm{H}^{1} \mathrm{fHGC}_{-1, n}$ & "pushing the hair" & 5.3 \\
\hline$D^{(2)}$ & $\mathrm{H}^{2} \mathrm{fHGC}_{-1,0} \rightarrow \mathrm{H}^{0} \mathrm{fHGC}_{-1,0}$ & "deleting the flower" & 2.19 \\
\hline$\chi^{1}$ & fHGC $_{-1, n} \rightarrow \mathrm{fHGC}_{-1, n}$ & adding a hair & 2.20 \\
\hline$\chi^{d}$ & fHGC $_{-1,0} \rightarrow \mathrm{fHGC}_{-1,0}$ & adding $d$ hairs & 2.21 \\
\hline$c$ & fHGC $_{-1,0} \rightarrow \mathrm{fHGC}_{-1,0}$ & transforming a hair into an antenna \\
\hline
\end{tabular}

Table 2 The table of differentials and important maps between graph complexes.

\begin{tabular}{|c|l|}
\hline Prefix & Explanation \\
\hline $\mathrm{V}^{k}$ & Graphs with number of vertices $v=k$ \\
\hline $\mathrm{E}^{k}$ & Graphs with number of edges $e=k$ \\
\hline $\mathrm{H}^{k}$ & Graphs with number of hairs $h=k$ \\
\hline $\mathrm{C}^{k}$ & Graphs with number of connected components $c=k$ \\
\hline $\mathrm{B}^{k}$ & Graphs with $e-v=k$ \\
\hline $\mathrm{A}^{k}$ & Graphs with $e+h=k$ \\
\hline $\mathrm{F}^{k}$ & Graphs with $e+h-v=k$ \\
\hline $\mathrm{P}^{k}$ & Graphs with number of tree-like connected components $p=k$ \\
\hline
\end{tabular}

Table 3 The table of prefixes that determine certain subcomplexes of a complex. The prefixes mean the subcomplex of the complex that follows, spanned by graphs with the fixed number stated.

Some differentials do not change some numbers from the above, so the complex splits as a direct product of complexes with fixed that number, e.g.

$$
\begin{aligned}
\left(\mathrm{fGC}_{n}^{\geq i}, \delta\right) & =\prod_{b \in \mathbb{Z}}\left(\mathrm{B}^{b} \mathrm{fGC}_{n}^{\geq i}, \delta\right), \\
\left(\mathrm{fGC}_{n}^{\geq i}, \nabla\right) & =\prod_{v \in \mathbb{N}}\left(\mathrm{V}^{v} \mathrm{fGC}_{n}^{\geq i}, \nabla\right),
\end{aligned}
$$




$$
\begin{gathered}
\left(\mathrm{fHGC}_{-1, n}^{\geq i}, \delta\right)=\prod_{b \in \mathbb{Z}}\left(\mathrm{B}^{b} \mathrm{fHGC}_{-1, n}^{\geq i}, \delta\right)=\prod_{h \in \mathbb{N}}\left(\mathrm{H}^{h} \mathrm{fHGC}_{-1, n}^{\geq i}, \delta\right)=\prod_{b \in \mathbb{Z}} \prod_{h \in \mathbb{N}}\left(\mathrm{B}^{b} \mathrm{H}^{h} \mathrm{fHGC}_{-1, n}^{\geq i}, \delta\right), \\
\left(\mathrm{fHGC}_{-1, n}^{\geq i}, \Delta\right)=\prod_{v \in \mathbb{N}}\left(\mathrm{V}^{v} \mathrm{fHGC}_{-1, n}^{\geq i}, \Delta\right)=\prod_{a \in \mathbb{Z}}\left(\mathrm{A}^{a} \mathrm{fHGC}_{-1, n}^{\geq i}, \Delta\right)=\prod_{v \in \mathbb{N}} \prod_{a \in \mathbb{Z}}\left(\mathrm{A}^{a} \mathrm{~V}^{v} \mathrm{fHGC}_{-1, n}^{\geq i}, \Delta\right), \\
\left(\mathrm{fHGC}_{-1, n}^{\geq i}, \delta+\Delta\right)=\prod_{f \in \mathbb{Z}}\left(\mathrm{F}^{f} \mathrm{fHGC}_{-1, n}^{\geq i}, \delta+\Delta\right) .
\end{gathered}
$$

We also use superscripts in the prefix of the form of inequality, e.g. $\mathrm{V}^{\geq k}$, that obviously means the subcomplex spanned by graphs which fulfill the inequality, e.g.

$$
\mathrm{V}^{\geq v} \mathrm{C}=\prod_{k \geq v} \mathrm{~V}^{k} \mathrm{C} .
$$

We have already used this notation in (2.13). Those subcomplexes with inequality often form a filtration of the complex. To this filtration a spectral sequence is associated. We say that the spectral sequence is on the number given by the prefix. E.g. the spectral sequence of $\left(\mathrm{fGC}_{n}, \delta+\Delta\right)$ on the number of vertices is the one associated to the filtration $\left(\mathrm{V}^{\geq v} \mathrm{fGC}_{n}, \delta+\Delta\right)$. Note that the expression on the number does not include the data weather the differential can increase or decrease the number, and therefore which filtration is used, the one defined with $\geq$ or $\leq$. But in any given case it will be clear weather the differential increases or decreases the number. In this case it is $\geq v$, not $\leq v$, because the differential $\delta+\Delta$ can increase, but not decrease the number of vertices. Its first differential is clearly $\Delta$.

We say that a spectral sequence converges correctly if it converges to the cohomology of the whole complex. To ensure correct convergence standard spectral sequence arguments, i.e. those from [5. Appendix C], are used. For doing so, it is often useful for a complex to be finite dimensional in each degree. It is mostly not the case, but after splitting a complex as in $2.26-2.29$, each subcomplex often has that property. So, the spectral sequence arguments can be used for each of them, and so we can compute the cohomology of the whole complex.

The following superscript will also be used.

$$
\mathrm{B}^{\leq f, p a r} \mathrm{C}:=\prod_{i \geq 0} \mathrm{~B}^{f-2 i} \mathrm{C}, \quad \mathrm{B}^{<f, p a r} \mathrm{C}:=\prod_{i>0} \mathrm{~B}^{f-2 i} \mathrm{C}
$$

i.e. it is the product of subcomplexes with $e-v \leq f$, respectively $e-v<f$ of the same parity as $f$.

As explained in the appendix A we use the notation $\overline{\mathrm{V}}^{v} \mathrm{C}$ for the space similar to $\mathrm{V}^{v} \mathrm{C}$ but with distinguishable vertices, i.e. the space of coinvariants of other groups (permuting edges, hairs, etc.) before taking coinvariants of the symmetric group $S_{v}$ that permutes vertices. It holds that

$$
\mathrm{V}^{v} \mathrm{C}=\left(\overline{\mathrm{V}}^{v} \mathrm{C}\right)^{S_{v}} .
$$

Let $S_{v-1}$ act on $\overline{\mathrm{V}}^{v} \mathrm{C}$ as sub-action of $S_{v}$ that permutes the first $v-1$ vertices, leaving the last vertex. We define

$$
\dot{\mathrm{V}}^{v} \mathrm{C}:=\left(\overline{\mathrm{V}}^{v} \mathrm{C}\right)^{S_{v-1}} .
$$

We may need the total spaces

$$
\begin{aligned}
\overline{\mathrm{V}} \mathrm{C} & :=\prod_{v \geq 1} \overline{\mathrm{V}}^{v} \mathrm{C}, \\
\dot{\mathrm{V} C} & :=\prod_{v \geq 1} \dot{\mathrm{V}}^{v} \mathrm{C} .
\end{aligned}
$$

\section{Deleting a vertex in non-hairy graphs}

In this section we introduce a new operation $D$ on the non-hairy graph complexes $\mathrm{fGC}_{n}$ which we call "deleting a vertex". Under some weak conditions, it holds that $D^{2}=0$ and a grading can be settled such that $D$ is a differential. We also obtain one further result about the spectral sequence of [5] for $n=0$. The results will be used for the hairy graph complex in Section 7 . 


\subsection{Even case}

For a graph $\Gamma \in \mathrm{fGC}_{0}$ we call the set of its vertices $V(\Gamma)$ and define

$$
D(\Gamma):=\sum_{x \in V(\Gamma)} D_{x}(\Gamma)=\sum_{x \in V(\Gamma)}(-1)^{v(x)} \tilde{D}_{x}(\Gamma)
$$

where $v(x)$ is the valence of the vertex $x, \tilde{D}_{x}$ deletes the vertex $x$ and sums over all ways of reconnecting edges that were connected to $x$ to the other vertices, skipping graphs with a tadpole, and $D_{x}=(-1)^{v(x)} \tilde{D}_{x}$. An example is sketched in Figure 4

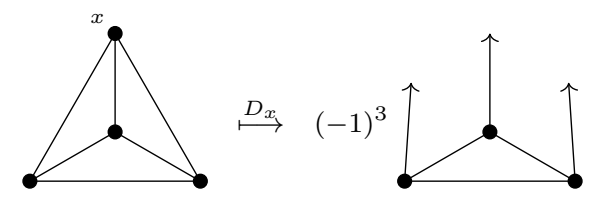

Fig. 4 Example of the action $D_{x}$, deleting the vertex $x$. The graph at the right means the sum over all graphs that can be formed by attaching ends of arrows to vertices, without making a tadpole.

We can restrict $D$ to $\mathrm{fGC}_{0}^{\geq 1}$ and $\mathrm{fGC}_{0}^{\geq 2}$. The following propositions about $D$ are stated in the broadest space possible, i.e. in $\mathrm{fGC}_{0}^{\geq i}$ for the smallest $i$ where it holds.

Proposition 3.1. In $\mathrm{fGC}_{0}^{\geq 2}$ it holds that

$$
D^{2}=0
$$

Proof. For a graph $\Gamma$

$$
D^{2}(\Gamma)=\sum_{\substack{x, y \in V(\Gamma) \\ x \neq y}} D_{y} D_{x}(\Gamma) .
$$

We now fix $x, y \in V(\Gamma), x \neq y$. Since $D$ does not change the number of edges, we can distinguish them, see Appendix A. Recall that for even $n$ there are no multiple edges in a graph. Vertices of $\Gamma \in \mathrm{fGC}_{0}^{\geq 2}$ are at least 2 -valent, so there exist an edge between $x$ and another vertex that is not $y$. We choose one of them and call it $f$.

$D_{y} D_{x}$ first deletes vertex $x$, reconnects its edges to other vertices, deletes vertex $y$ and reconnects its edges, including those that came from $x$, to other vertices. Let us fix one way of reconnecting edges that are not $f$ and the final destination of $f$. For that way of reconnecting, in $D_{y} D_{x}$ there are two terms: the one where $f$ goes directly to its final destination with $D_{x}$ and the one where $f$ goes to $y$ with $D_{x}$ and to its final destination with $D_{y}$. In the later the valence of $y$ while applying $D_{y}$ is by 1 bigger than its valence while applying $D_{y}$ in the former term, making the terms cancel each other because of the sign dependence on valence. It follows that $D_{y} D_{x}(\Gamma)=0$ and therefore $D^{2}(\Gamma)=0$.

Proposition 3.2. In $\mathrm{fGC}_{0}^{\geq 1}$ it holds that

$$
D^{4}=0
$$

Proof. From the previous proof we see that $D_{y} D_{x}(\Gamma)$ may only be non-zero if $x$ is 1 -valent vertex with an edge connecting to $y$.

So $D_{z} D_{y} D_{x}(\Gamma) \neq 0$ implies $x$ is of that kind. But also $D_{z} D_{y}\left(D_{x}(\Gamma)\right) \neq 0$ implies $y$ is 1 -valent and connected to $z$ in $D_{x}(\Gamma)$. Since $D_{x}$ did not change the valence of $y$, already in $\Gamma$ it was 1 -valent, and vertices $x$ and $y$ formed $\lambda=$

Now let us pick a term in $D^{4}(\Gamma)$, say $D_{w} D_{z} D_{y} D_{x}(\Gamma)$. It being non-zero implies $D_{z} D_{y} D_{x}(\Gamma) \neq 0$, so $x$ and $y$ form $\lambda$. Similarly, $D_{w} D_{z} D_{y}\left(D_{x}(\Gamma)\right) \neq 0$ imply $y$ and $z$ form $\lambda$ in $D_{x}(\Gamma)$. Since the edge at $z$ came from $x, z$ was isolated in $\Gamma$ what is not possible in $\mathrm{fGC}_{0}^{\geq 1}$. Therefore $D_{w} D_{z} D_{y} D_{x}(\Gamma)$ is always 0 and $D^{4}=0$.

Recall the extra differential $\nabla: \mathrm{fGC}_{0} \rightarrow \mathrm{fGC}_{0}$ that acts by adding one edge in all possible ways defined in [5] and cited in 2.3 . 
Proposition 3.3. In $\mathrm{fGC}_{0}$ it holds that

$$
\delta D-D \delta=\nabla
$$

Proof.

$$
\begin{gathered}
(\delta D(\Gamma)-D \delta(\Gamma))=\delta\left(\sum_{x} D_{x}(\Gamma)\right)-D\left(\sum_{y} \frac{1}{2} s_{y}(\Gamma)-a_{y}(\Gamma)\right)=\sum_{x} \sum_{\substack{y \\
y \neq x}}\left(\frac{1}{2} s_{y} D_{x}(\Gamma)-a_{y} D_{x}(\Gamma)\right) \\
-\frac{1}{2} \sum_{y}\left(\sum_{\substack{x \\
x \neq y}} D_{x} s_{y}(\Gamma)+D_{y} s_{y}(\Gamma)+D_{z} s_{y}(\Gamma)\right)+\sum_{y}\left(\sum_{\substack{x \\
x \neq y}} D_{x} a_{y}(\Gamma)+D_{y} a_{y}(\Gamma)+D_{z} a_{y}(\Gamma)\right)= \\
\frac{1}{2} \sum_{\substack{x, y \\
x \neq y}} s_{y} D_{x}(\Gamma)-\sum_{\substack{x, y \\
x \neq y}} a_{y} D_{x}(\Gamma)-\frac{1}{2} \sum_{\substack{x, y \\
x \neq y}} D_{x} s_{y}(\Gamma)-\sum_{x} D_{z} s_{x}(\Gamma)+\sum_{\substack{x, y \\
x \neq y}} D_{x} a_{y}(\Gamma)+\sum_{x} D_{x} a_{x}(\Gamma)+\sum_{x} D_{z} a_{x}(\Gamma),
\end{gathered}
$$

where $x$ and $y$ run through $V(\Gamma)$ and $z$ is the name of the newly added vertex.

For different $x, y \in V(\Gamma)$ it easily follows that

$$
s_{y} D_{x}(\Gamma)=D_{x} s_{y}(\Gamma) .
$$

The term $D_{z} s_{x}(\Gamma)$ first splits a new vertex $z$ from the vertex $x$, connects them with an edge, say $g$, and reconnects some of the edges from $x$ to $z$. Afterwards it deletes vertex $z$ and reconnects edges from it to other vertices, possibly back to $x$, and reconnects $g$ also to other vertex. The final result is that some of the edges are reconnected from $x$ to other vertices, and there is a new edge $g$ connecting $x$ and some other vertex. The edge $g$ can be seen as added at the end, and before that, since number of edges is not changed, we can distinguish edges. Suppose there is an edge that at the end stays at $x$, and call it $f$. We fix one way of reconnecting all other edges. For that way of reconnecting there are two terms in $D_{z} s_{x}(\Gamma)$ : one where $f$ stays at $x$ and one where it is reconnected to $z$ and back to $x$, and they cancel each other because valence of $z$ differ by one in them. Therefore everything what survives from $D_{z} s_{x}(\Gamma)$ is reconnecting all edges from $x$ to other vertices and adding an edge $g$ from $x$ to some other vertex, say $y$. That is exactly the same as deleting vertex $x$ and adding an edge at $y$, with the opposite sign because the valence of the vertex being deleted differs by one. So we get

$$
D_{z} s_{x}(\Gamma)=-\sum_{\substack{y \\ y \neq x}} a_{y} D_{x}(\Gamma) .
$$

An easy argument, that is left to the reader, implies

$$
D_{x} a_{x}(\Gamma)=-\sum_{\substack{y \\ y \neq x}} D_{x} a_{y}(\Gamma) .
$$

The last term $\sum_{x} D_{z} a_{x}(\Gamma)$ clearly adds an edge from $x$ to some other vertex, so it holds that

$$
\sum_{x} D_{z} a_{x}(\Gamma)=\nabla(\Gamma)
$$

By equations obtained, all except the last term of the above expression cancel, and the claimed formula follows.

Propositions 3.1 and 3.3 easily imply $D \nabla+\nabla D=0$ in $\mathrm{fGC}_{0}^{\geq 2}$. But we need a bit stronger result.

Proposition 3.4. In $\mathrm{fGC}_{0}^{\geq 1}$ it holds that

$$
D \nabla+\nabla D=0 .
$$

Proof. $D \nabla$ puts an edge in all possible ways and then deletes a vertex, say $x$. If the new edge has been connected to $x$, it is moved to another vertex. Let finally the new edge connect vertices $y$ and $z$. To that position it can come in three different ways: 
- directly been connected to $y$ and $z$ by $\nabla$, what is exactly the corresponding term from $\nabla D$;

- been connected from $y$ to $x$ by $\nabla$ and then moved to $z$ by $D_{x}$, what is the negative of the term in $\nabla D$ because of the sign changes in deleting vertex $x$ with one more valence;

- and the same from $z$ to $x$, what is also the negative of the term in $\nabla D$.

All terms in $\nabla D$ are come like this, so indeed $D \nabla=\nabla D-2 \nabla D=-\nabla D$.

Proposition 3.5. In $\mathrm{fGC}_{0}^{\geq 1}$ it holds that

$$
\nabla D^{2}=0
$$

Proof. From the proof of Proposition 3.1 the term $D_{y} D_{x}(\Gamma)$ of $D^{2}(\Gamma)$ may only be non-zero if $x$ is a 1 -valent vertex with an edge connecting to $y$. Then $D_{y} D_{x}$ deletes $x, y$ and the edge between them, reconnects all other edges from $y$ elsewhere and adds an edge in all possible ways. Then $\nabla$ adds another edge in all possible ways. Adding one edge on one place and another edge on another place cancels with adding edges in the opposite order, hence the result.

\subsection{Odd case}

Like in the even case, for a graph $\Gamma \in \mathrm{fGC}_{1}$ we define

$$
D(\Gamma):=\sum_{x \in V(\Gamma)} D_{x}=\sum_{x \in V(\Gamma)}(-1)^{v(x)} \tilde{D}_{x} .
$$

Note that multiple edges are now possible. Similarly as with splitting, if an $N$-fold edge went to the vertex being deleted, there is a factor $\left(\begin{array}{c}N \\ k_{1}, k_{2}, \ldots\end{array}\right)$ where $k_{i}$ is the number of edges from that multiple edge that go to the vertex $i$. We can restrict $D$ to $\mathrm{fGC}_{1}^{\geq 1}$.

Proposition 3.6. On $\mathrm{fGC}_{1}^{\geq 1}$ it holds that

$$
D^{2}=0
$$

Proof. Like in the proof of Proposition 3.1 we write

$$
D^{2}(\Gamma)=\sum_{\substack{x, y \in V(\Gamma) \\ x \neq y}} D_{y} D_{x}(\Gamma)
$$

fix $x, y \in V(\Gamma)$ and distinguish edges. If there is an edge from $x$ to a vertex other than $y$ the same reasoning from Proposition 3.1 leads to $D_{y} D_{x}(\Gamma)=0$. If not, all edges from $x$ go to $y$ and let there be $k>0$ of them. Let $\Gamma^{\prime}$ be the graph obtained from $\Gamma$ by deleting vertex $x$ and all $k$ edges at $x$. Then $D_{y} D_{x}(\Gamma)$ is actually $D_{y}\left(\Gamma^{\prime}\right)$ where we add $k$ edges in all possible ways. But adding an edge from one vertex to another cancels with adding an opposite edge, leading to the conclusion $D_{y} D_{x}(\Gamma)=0$. Therefore it again holds that $D^{2}=0$.

Proposition 3.7. On $\mathrm{fGC}_{1}$ it holds that

$$
\delta D+D \delta=0
$$

Proof. The argument, with a bit of care for the signs, is the same as in the proof of Proposition 3.3 . Only in the last term adding an edge from one vertex to another cancels with adding the opposite one, leading to the result 0 . 
3.3 More about the spectral sequence of [5] for the even case

Recall from [5, Corollary 4] that $\mathcal{H}\left(\mathrm{fGCc}_{0}, \delta+\nabla\right)=0$ and that there is a spectral sequence converging to it whose $E^{1}$ term (i.e. the first page) is $\mathcal{H}\left(\mathrm{fGCc}_{0}, \delta\right)$ with the differential $\nabla$. The spectral sequence is on the number $b=e-v$. The following corollaries calculate homologies of some similar complexes.

Corollary 3.8. The complex $\left(\mathrm{fGCc}_{0}^{\geq 2}, \delta+\nabla\right)$ is acyclic.

Proof. On the mapping cone of the inclusion $\left(\mathrm{fGCc}_{0}^{\geq 2}, \delta+\nabla\right) \hookrightarrow\left(\mathrm{fGCc}_{0}, \delta+\nabla\right)$ we set up a spectral sequence mentioned above, on the number $b=e-v$. The complex with the first differential is the mapping cone of the inclusion $\left(\mathrm{fGCc}_{0}^{\geq 2}, \delta\right) \hookrightarrow\left(\mathrm{fGCc}_{0}, \delta\right)$. It is acyclic by Proposition 2.1 The spectral sequence converges correctly because the space in each degree $e$ is finitely dimensional, so the whole mapping cone is acyclic. That leads to $\mathcal{H}\left(\mathrm{fGCc}_{0}^{\geq 2}, \delta+\nabla\right)=\mathcal{H}\left(\mathrm{fGCc}_{0}, \delta+\nabla\right)=0$.

Corollary 3.9. The complex $\left(\mathrm{fGC}_{0}^{\geq 2}, \delta+\nabla\right)$ is acyclic.

Proof. On $\left(\mathrm{fGC}_{0}^{\geq 2}, \delta+\nabla\right)$ we set up a spectral sequence on the number of connected components. It clearly converges to the cohomology of the whole complex. In the $c$-th row there is a complex

$$
\left(\left(\mathrm{fGCc}_{0}^{\geq 2}, \delta+\nabla\right)^{\otimes c}\right)^{S_{c}}[1-c]
$$

that is acyclic by (1), hence the result.

On $\mathrm{fGC}_{0}^{\geq 1}$ let us conjugate the differential $\delta+\nabla$ to $e^{D}(\delta+\nabla) e^{-D}$. We have the following proposition. Proposition 3.10. In $\mathrm{fGC}_{0}^{\geq 1}$ it holds that

$$
e^{D}(\delta+\nabla) e^{-D}=\delta+D \nabla .
$$

Proof. Using results of Propositions 3.2 to 3.5 it holds that

$$
\begin{aligned}
e^{D}(\delta+\nabla) e^{-D} & =\left(\mathrm{Id}+D+\frac{D^{2}}{2}+\frac{D^{3}}{6}\right)(\delta+\nabla)\left(\mathrm{Id}-D+\frac{D^{2}}{2}-\frac{D^{3}}{6}\right)= \\
= & \delta+\nabla+D \delta+D \nabla-\delta D-\nabla D-D \delta D+\frac{D^{2} \delta}{2}-\frac{D^{2} \delta D}{2}+\frac{\delta D^{2}}{2}+\frac{D \delta D^{2}}{2}=\delta+D \nabla .
\end{aligned}
$$

Note that conjugated differential $\delta+D \nabla$ can not change $b=e-v$ by an odd amount. Therefore complexes with that differential split into the direct sum of two complexes, one with even and one with odd $b$. The following result is now straightforward.

Corollary 3.11. There is a spectral sequence converging to

$$
\mathcal{H}\left(\mathrm{fGC}_{0}^{\geq 2}, \delta+D \nabla\right)=0
$$

whose first page is

$$
\mathcal{H}\left(\mathrm{fGC}_{0}^{\geq 2}, \delta\right)
$$

Furthermore, in this spectral sequence, differentials on odd pages are 0.

The corollary is similar to [5, Corollary 4], but this time the complex includes disconnected graphs. Indeed, 5. Corollary 4] implies the same result for disconnected graphs, and in this sense our result is weaker. There is the filtration on $b=e-v$ in both cases, but different total differentials $\delta+\nabla$ and $\delta+D \nabla$, that are both acyclic. So, in both cases there are cancellations of classes in $\mathcal{H}\left(\mathrm{fGC}_{0}^{\geq 2}, \delta\right)$ as drawn in Table 4 For disconnected graphs our result is stronger because we know that there are no cancellations on odd pages. An example of cancelling with our differential $\delta+D \nabla$ is depicted in Figure 5. c.f. cancelling of the same classes with the differential $\delta+\nabla$ in [5, Figure 2]. 


\begin{tabular}{|c|c|c|c|c|c|c|c|c|c|c|c|c|c|c|c|c|c|c|c|c|c|c|c|c|c|c|c|c|c|}
\hline & 0 & 1 & 2 & 3 & 4 & 5 & 6 & & & & & 0 & 11 & 12 & 13 & 14 & 15 & 16 & 17 & 18 & 19 & 20 & 21 & 22 & 23 & 2 & 2 & 26 & 27 \\
\hline-1 & 0 & 0 & 0 & 0 & 0 & 0 & 0 & & c & & 0 & 0 & 0 & 0 & 0 & 0 & 0 & 0 & 0 & 0 & 0 & 0 & 0 & 0 & 0 & 0 & 0 & 0 & 0 \\
\hline 0 & & 0 & 0 & 0 & 0 & 1 & 0 & ( & ( & & 1 & 0 & 0 & 0 & 1 & 1 & 0 & 0 & 1 & 1 & 0 & 0 & 1 & 2 & 0 & 0 & 1 & 2 & 1 \\
\hline 1 & & & 0 & 0 & 0 & 0 & $\downarrow 0$ & ( & ( & & 0 & 0 & 0 & 0 & of & 0 & 0 & 0 & 0 & 0 & 0 & 0 & 0 & 0 & 0 & 0 & 0 & 0 & 0 \\
\hline 2 & & & & 0 & 0 & 0 & 1 & & ( & & 0 & 0 & 1 & 0 & 0 & 0 & 1 & 0 & 0 & 0 & 1 & 1 & 0 & 0 & 0 & 1 & 1 & 0 & 0 \\
\hline 3 & & & & & 0 & 0 & 0 & ( & ( & & 0 & 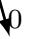 & 0 & 0 & 0 & 0 & 0 & 0 & 0 & 0 & 0 & 0 & 0 & 0 & 0 & 0 & 0 & 0 & 0 \\
\hline 4 & & & & & & 0 & 0 & & ( & & 0 & 1 & 0 & 1 & 0 & 0 & 1 & 0 & 1 & 0 & 1 & 0 & 1 & 0 & 1 & 1 & 1 & 1 & 0 \\
\hline 5 & & & & & & & 0 & & ( & & 0 & 0 & 0 & 0 & 0 & 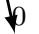 & 1 & 0 & 0 & 0 & 0 & 1 & 0 & 0 & 0 & 1 & 0 & 0 & 0 \\
\hline 6 & & & & & & & & ( & ( & & 0 & 0 & 0 & 0 & 0 & 1 & 0 & 1 & 0 & 1 & 1 & 0 & 1 & 0 & 2 & 0 & 1 & 0 & 2 \\
\hline 7 & & & & & & & & & ( & & 0 & 0 & 0 & 0 & 0 & 0 & 0 & 1 & 0 & 0 & 1 & 0 & 2 & 0 & 0 & 1 & 1 & 1 & 0 \\
\hline 8 & & & & & & & & & & & 0 & 0 & 0 & 0 & 0 & 0 & 0 & 0 & 0 & 1 & 0 & 2 & 1 & 1 & 1 & 1 & 2 & 1 & 2 \\
\hline 9 & & & & & & & & & & & & 0 & 0 & 0 & 0 & 0 & 0 & 0 & 0 & 0 & 0 & 1 & 0 & 1 & 2 & 0 & 3 & 0 & 3 \\
\hline 10 & & & & & & & & & & & & & 0 & 0 & 0 & 0 & 0 & 0 & 0 & 0 & 0 & 0 & 0 & 2 & 0 & $?$ & $?$ & $?$ & $?$ \\
\hline 11 & & & & & & & & & & & & & & 0 & 0 & 0 & 0 & 0 & 0 & 0 & 0 & 0 & 0 & 0 & 0 & 2 & $?$ & $?$ & $?$ \\
\hline 12 & & & & & & & & & & & & & & & 0 & 0 & 0 & 0 & 0 & 0 & 0 & 0 & 0 & 0 & 0 & 0 & 0 & 3 & ? \\
\hline
\end{tabular}

Table 4 Table of dimensions of cohomology $\mathcal{H}\left(\mathrm{fGC}_{0}, \delta\right)$. The column number represents the number of edges $e$ and the row number represents $b=e-v$. Known cancellations are depicted by arrows.

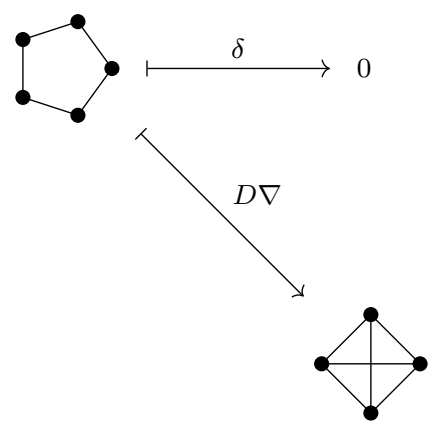

Fig. 5 The first cancelling from Table 4 A loop cocyle cancels another graph cocyle in the spectral sequence, c.f. cancelling of the same classes with the differential $\delta+\nabla$ in [5] Figure 2].

\section{Constraints on hairy graphs}

In this section we study subcomplexes of Hairy graph complex spanned by graphs that fulfil certain constraints. Some of them are standard simplifications of graph complexes by restricting the minimal valence of vertices, cf. [19, Proposition 3.4]. The others seem technical, like disallowing certain small graphs to be a connected component (constraints $\dagger$ and $\ddagger$ ). We need all that complexes for the final proofs whose strategies are explained at the beginnings of Sections 6 and 7

4.1 Simplifying the full graph complex

Proposition 2.1 ([19, Proposition 3.4]) can easily be extended to the hairy case as follows.

Proposition 4.1. The cohomologies satisfy

$$
\mathcal{H}\left(\mathrm{fHGCc}_{-1, n}^{\geq 0}, \delta\right)=\mathcal{H}\left(\mathrm{fHGCc}_{-1, n}^{\geq 2}, \delta\right)=\mathcal{H}\left(\mathrm{fHGCc}_{-1, n}^{\geq 3}, \delta\right) \oplus \bigoplus_{\substack{j \geq 3 \\ j \equiv 2 n+1}} \mathbb{K o d} 4
$$

Proof. The same argument as in [19, Proposition 3.4]. Roughly, we construct subcomplex fHGCc $_{-1, n}^{1}$ of HHGCc $_{-1, n}^{\geq 0}$ spanned by graphs with at least one 1-valent vertices (including one vertex graph $\sigma=$ $\sigma_{0}=\bullet$ ). Graphs in that complex have "antennas", that are parts that end with a 1-valent vertex. One can make a spectral sequence whose first differential acts only on antennas, and it is easily shown that the complex with that differential is acyclic, hence $\left(\mathrm{fHGCc}_{-1, n}^{1}, \delta\right)$ is acyclic. Since $\mathrm{fHGCc}_{-1, n}^{\geq 0}=$ $\mathrm{fHGCc}_{-1, n}^{\geq 2} \oplus \mathrm{fHGCc}_{-1, n}^{1}$, this shows the first equality. 
Next, let $\mathrm{fHGCc}_{-1, n}^{2}$ be subcomplex of $\mathrm{fHGCc}_{-1, n}^{\geq 2}$ with at least one 2 -valent vertex. It contains loops (graphs with no hairs and only 2 -valent vertices) whose cohomology is easily seen to be

$$
\bigoplus_{\substack{j \geq 3 \\ j \equiv 2 n+1}} \mathbb{K}[-1-j] .
$$

On the rest we can make a spectral sequence with the first differential extending the chain of edges and 2 -valent vertices between non-2-valent or hairy vertices. It is again easily shown that the complex with that differential is acyclic. Since fHGCc${ }_{-1, n}^{\geq 2}=\mathrm{fHGCc}_{-1, n}^{\geq 3} \oplus \mathrm{fHGCc}_{-1, n}^{2}$, the second equality follows.

Recall that the standard valence constraint for hairy complexes in this paper is $\geq 1$, i.e. the notation fHGCc means $\mathrm{fHGCc}^{\geq 1}$, see (2.16). But that constraint is exactly the one not mentioned in Proposition 4.1. Luckily, in subspace fHGCc $\subset$ fHGCc ${ }^{\geq 0}$ only one graph is excluded, the one vertex graph $\sigma=\sigma_{0}=\bullet$. This makes the cohomology $\mathcal{H}\left(\mathrm{fHGCc}_{-1, n}, \delta\right)$ different from $\mathcal{H}\left(\mathrm{fHGCc}_{-1, n}^{\geq 0}, \delta\right)=\mathcal{H}\left(\mathrm{fHGCc}_{-1, n}^{\geq 2}, \delta\right)$ by one class. The class is represented by $\lambda=\lambda_{1}=\bullet \bullet$. If this graph is excluded too, the resulting complex will again be quasi-isomorphic to $\left(\mathrm{fHGCc}_{-1, n}^{\geq 0}, \delta\right)$. In the next definition we define that complex, and in the following lemma we state the result. Although we work with connected graphs now, the definition is already made suitable for disconnected graphs.

Definition 4.2. Let $\mathrm{fHGC}_{-1, n}^{\dagger} \subset \mathrm{fHGC}_{-1, n}$ be the subspace spanned by graphs that do not have $\lambda$ as a connected component. Let

$$
\mathrm{fGC}_{n}^{\dagger}:=\mathrm{H}^{0} \mathrm{fHGC}_{-1, n}^{\dagger}[-1-n] \subset \mathrm{fGC}_{n}^{\geq 1}
$$

c.f. (2.11).

Lemma 4.3. Space HHGCc $_{-1, n}^{\dagger}$ is closed under differential $\delta$ and the cohomologies satisfy

$$
\mathcal{H}\left(\mathrm{fHGCc}_{-1, n}^{\geq 0}, \delta\right)=\mathcal{H}\left(\mathrm{fHGCc}_{-1, n}^{\dagger}, \delta\right) .
$$

Proof. The discussion above implies the result.

The following constraint is motivated as follows, c.f. proof of Proposition 6.8 In the hairy complexes with the extra differential $\left(\mathrm{fHGC}_{-1, n}, \delta+\Delta\right)$ the number of hairs $h$ is unbounded. That makes the spectral sequences on $h$ (that has $\delta$ as the first differential) and the one on the number of connected components $c$ unbounded from above and we can not use standard results for convergence.

The complex splits as in 2.30$)$ :

$$
\left(\mathrm{fHGC}_{-1, n}, \delta+\Delta\right)=\prod_{f \in \mathbb{Z}}\left(\mathrm{F}^{f} \mathrm{fHGC}_{-1, n}, \delta+\Delta\right)
$$

where $f=e+h-v$. In every $\mathrm{F}^{f} \mathrm{fHGC}_{-1, n}$ for the fixed degree $d=1+v n+(1-n) e-n h$, the number of edges $e=d+n f-1$ is fixed too. So, increasing the number of hairs $h$ increases the number of vertices $v$ by the same amount. Since $e$ is fixed, that increase will eventually force a graph to have connected components that are stars $\sigma_{1}=\boldsymbol{\bullet}$. In the next subsection we will show that components $\sigma_{1}$ are mostly irrelevant for the cohomology of the deformed complex, so we can disallow them and bound $h$ from above while preserving the result about cohomology. Now we define the complex without $\sigma_{1}$, and also without $\lambda_{2}=\bullet$ to preserve the cohomology with the standard differential, as stated in the following lemma. Because of its importance, we have a separate name for this complex, the bounded graph complex.

Definition 4.4. Let the bounded graph complex $\mathrm{HHGC}_{-1, n}^{\ddagger} \subset \mathrm{fHGC}_{-1, n}^{\dagger}$ be the subspace spanned by graphs that do not have $\lambda, \sigma_{1}=\bullet$ or $\lambda_{2}=\bullet \bullet \bullet$ as a connected component.

Recall that Example 2.3 implies in particular that $\delta\left(\sigma_{1}\right)=\lambda_{2}$.

Lemma 4.5. Space $\mathrm{fHGC}_{-1, n}^{\ddagger}$ is closed under the differential $\delta$ and the cohomologies satisfy

$$
\mathcal{H}\left(\mathrm{fHGCc}_{-1, n}^{\dagger}, \delta\right)=\mathcal{H}\left(\mathrm{fHGCc}_{-1, n}^{\ddagger}, \delta\right) .
$$


Proof. The new complex has two graphs less and they cancel each other by the differential, so it does not change the cohomology.

The following constraint is necessary for Lemma 5.2 used in the proof of Proposition 6.9

Definition 4.6. Let $\mathrm{fHGC}_{-1, n}^{\natural} \subset \mathrm{fHGC}_{-1, n}^{\geq 2}$ be the subspace spanned by graphs that do not have 2 -valent vertex with a hair.

Lemma 4.7. Space $\mathrm{fHGC}_{-1, n}^{\natural}$ is closed under differential $\delta$ and the cohomologies satisfy

$$
\mathcal{H}\left(\mathrm{fHGCc}_{-1, n}^{\geq 2}, \delta\right)=\mathcal{H}\left(\mathrm{fHGCc}_{-1, n}^{\natural}, \delta\right) .
$$

Proof. The idea of proving is similar to the one for Proposition 4.1 . It holds that $\mathrm{fHGCc}_{-1, n}^{\geq 2}=\mathrm{fHGCc}_{-1, n}^{\natural}$ $\oplus \mathrm{fHGCc}_{-1, n}^{/ \natural}$ where $\mathrm{fHGCc}_{-1, n}^{/ \natural}$ is the subspace of $\mathrm{HHGCc}_{-1, n}^{\geq 2}$ spanned by graphs with at least one 2valent vertex with a hair. Those graphs have "hairy antennas", i.e. parts that end with 2-valent vertex with one hair, or they are linear graphs with two 2-valent hairy vertices at the ends, including $\sigma_{2}=\bullet$. One can make a spectral sequence whose first differentials acts only on hairy antennas (extends the linear graphs respectively), and show that the complex with that differential is acyclic. This implies the result.

We systematize the results in the following proposition.

Proposition 4.8. The cohomologies satisfy

$\mathcal{H}\left(\mathrm{fHGCc}_{-1, n}^{\geq 0}, \delta\right)=\mathcal{H}\left(\mathrm{fHGCc}_{-1, n}^{\dagger}, \delta\right)=\mathcal{H}\left(\mathrm{fHGCc}_{-1, n}^{\ddagger}, \delta\right)=\mathcal{H}\left(\mathrm{fHGCc}_{-1, n}^{\geq 2}, \delta\right)=\mathcal{H}\left(\mathrm{fHGCc}_{-1, n}^{\natural}, \delta\right)$.

Proof. Follows from Proposition 4.1 and Lemmas $4.3,4.5$ and 4.7

The results can be extended to complexes that allow disconnected graphs as follows.

Corollary 4.9. All mentioned spaces are closed under differential $\delta$ and the cohomologies satisfy

$$
\mathcal{H}\left(\mathrm{fHGC}_{-1, n}^{\geq 0}, \delta\right)=\mathcal{H}\left(\mathrm{fHGC}_{-1, n}^{\dagger}, \delta\right)=\mathcal{H}\left(\mathrm{fHGC}_{-1, n}^{\ddagger}, \delta\right)=\mathcal{H}\left(\mathrm{fHGC}_{-1, n}^{\geq 2}, \delta\right)=\mathcal{H}\left(\mathrm{fHGC}_{-1, n}^{\natural}, \delta\right),
$$

Proof. The complexes are just the symmetric product of their connected parts, so the corollary directly follows from the proposition.

By Proposition 4.1 all classes missing in "3-valent complex" are in hairless part, so Proposition 4.8 also implies the following corollary. Recall that $\mathrm{HGC}_{-1, n}=\mathrm{H}^{\geq 1} \mathrm{fHGCc}_{-1, n}^{\geq 3}$.

Corollary 4.10. All mentioned spaces are closed under differential $\delta$ and the cohomologies satisfy

$$
\begin{aligned}
& \mathcal{H}\left(\mathrm{H}^{\geq 1} \mathrm{fHGCc}_{-1, n}^{\geq 0}, \delta\right)=\mathcal{H}\left(\mathrm{H}^{\geq 1} \mathrm{fHGCc}_{-1, n}, \delta\right)=\mathcal{H}\left(\mathrm{H}^{\geq 1} \mathrm{fHGCc}_{-1, n}^{\dagger}, \delta\right)= \\
& \quad=\mathcal{H}\left(\mathrm{H}^{\geq 1} \mathrm{fHGCc}_{-1, n}^{\ddagger}, \delta\right)=\mathcal{H}\left(\mathrm{H}^{\geq 1} \mathrm{fHGCc}_{-1, n}^{\geq 2}, \delta\right)=\mathcal{H}\left(\mathrm{H}^{\geq 1} \mathrm{fHGCc}_{-1, n}^{\natural}, \delta\right)=\mathcal{H}\left(\mathrm{HGC}_{-1, n}, \delta\right) .
\end{aligned}
$$

Proof. The differential $\delta$ does not change the number of hairs $h$, so previous results can be easily restricted to $\mathrm{H}^{\geq 1}$. Namely, Proposition 4.1 implies that the first, the fifth and the seventh term are equal, since the extra loop peace lives in hairless $h=0$ space.

Similarly, Proposition 4.8 implies that the first, the third, the forth, the fifth and the sixth terms are equal.

Note that first three complexes $\left(\mathrm{H}^{\geq 1} \mathrm{fHGCc}_{-1, n}^{\geq 0}, \delta\right),\left(\mathrm{H}^{\geq 1} \mathrm{fHGCc}_{-1, n}, \delta\right),\left(\mathrm{H}^{\geq 1} \mathrm{fHGCc}_{-1, n}^{\dagger}, \delta\right)$ are equal already as complexes.

All this together concludes the corollary.

For $n=0$ the $b=e-v=-1$ part of the cohomology is almost zero, as stated in the following lemma.

Lemma 4.11 ([1, Proposition 3.3], [17, Theorem 3]). $\mathcal{H}\left(\mathrm{B}^{-1} \mathrm{HGC}_{-1,0}, \delta\right)$ is 1-dimensional, the class being represented by the star $\sigma_{3}=\boldsymbol{\alpha}$. 
4.2 The bounded graph complex with the extra differential

As already announced, in this subsection we show that components $\sigma_{1}$ are mostly irrelevant for the cohomology of $\left(\mathrm{fHGC}_{-1, n}, \delta+\Delta\right)$, so we can switch to bounded complex while preserving the result about cohomology. Check that bounded space $\mathrm{fHGC}_{-1, n}^{\ddagger}$ is closed under the extra differential $\Delta$.

We will actually prove that the quotient between full complex and bounded complex, defined as follows, is almost acyclic.

Definition 4.12. The unbounded hairy graph complex $\mathrm{fHGC}_{-1, n}^{\dagger / \ddagger} \subset \mathrm{fHGC}_{-1, n}^{\dagger}$ is the subspace spanned by graphs that have at least one connected component $\sigma_{1}$ or $\lambda_{2}$, i.e. it is the quotient $\mathrm{fHGC}_{-1, n}^{\dagger} / \mathrm{fHGC}_{-1, n}^{\ddagger}$.

The unbounded remainder $\mathrm{UR}_{-1, n} \subset \mathrm{fHGC}_{-1, n}^{\dagger}$ is the subspace spanned by graphs with all connected components $\sigma_{1}$ or $\lambda_{2}$.

Although the unbounded graph complex $\mathrm{fHGC}_{-1, n}^{\dagger / \ddagger}$ is closed under the standard differential $\delta$, it is not closed under the extra differential $\Delta$. However, its complements, bounded graph complex is closed under $\Delta$. Therefore we can talk about complex $\left(\mathrm{fHGC}_{-1, n}^{\dagger / \ddagger}, \delta+\Delta\right)$ and understand that forbidden graphs are identified to zero. Formally, this complex is the quotient $\left(\mathrm{fHGC}_{-1, n}^{\dagger}, \delta+\Delta\right) /\left(\mathrm{fHGC}_{-1, n}^{\ddagger}, \delta+\Delta\right)$.

Note that because of symmetry reasons, there can be at most one connected component $\lambda_{2}=\bullet \bullet$ in a graph for both parities of $n$. Also, check that unbounded remainder $\mathrm{UR}_{-1, n}$ is closed under $\delta$ and $\Delta$.

Lemma B.1 from the appendix implies that cohomology $\mathcal{H}\left(\mathrm{UR}_{-1, n}, \delta+\Delta\right)$ is one-dimensional, the class being represented by

$$
\alpha=\sum_{n \geq 1} \frac{1}{n !} \sigma_{1}^{\cup n} .
$$

Proposition 4.13. $\mathcal{H}\left(\mathrm{fHGC}_{-1, n}^{\dagger / \ddagger}, \delta+\Delta\right)$ is one-dimensional, the class being represented by $\alpha$.

Proof. Let $\tilde{\Gamma} \in \mathrm{fHGC}_{-1, n}^{\dagger / \ddagger}$ be a graph. It may be either $\tilde{\Gamma} \in \mathrm{UR}_{-1, n}$ or we can write $\tilde{\Gamma}=\Gamma \cup \gamma$ where $\Gamma \in \mathrm{fHGC}_{-1, n}^{\ddagger}$ and $\gamma \in \mathrm{UR}_{-1, n}$. In the latter case we call $\Gamma$ the main part of $\tilde{\Gamma}$ and $\gamma$ the secondary part of $\tilde{\Gamma}$. In the former case the whole graph is the secondary part, while the main part is empty.

Let us set up a spectral sequence of $\left(\mathrm{fHGC}_{-1, n}^{\dagger / \ddagger}, \delta+\Delta\right)$ on the number of edges in the main part, empty main part having 0 edges. It is easily seen that the differential can not decrease that number. To ensure the correct convergence we split unbounded complex similarly as the full complex:

$$
\left(\mathrm{fHGC}_{-1, n}^{\dagger / \ddagger}, \delta+\Delta\right)=\prod_{f \in \mathbb{Z}}\left(\mathrm{F}^{f} \mathrm{fHGC}_{-1, n}^{\dagger / \ddagger}, \delta+\Delta\right) .
$$

In every $\mathrm{F}^{f} \mathrm{fHGC}_{-1, n}^{\dagger / \ddagger}$ for the fixed degree $d=1+v n+(1-n) e-n h$, the total number of edges $e=d+n f-1$ is fixed too, so the number of edges in the main part is bounded and therefore the spectral sequence converges correctly.

One can check that the first part of the differential is the one that acts within the secondary part only. Therefore the complexes on the first page are the direct product of complexes for the fixed main part, which are all clearly isomorphic to $\mathrm{UR}_{-1, n}$. Therefore, using Lemma B.1. on the first page of the spectral sequence we have classes represented by $\Gamma \cup \alpha$ for $\Gamma \in \mathrm{fHGC}_{-1, n}^{\ddagger}$, together with the class $[\alpha]$ itself.

On the second page matters the part of the differential that acts within the main part only, and the one that connects the main part to the secondary part. The element is now uniquely determined by the main part $\Gamma$ so we can investigate what does the differential do to it:

$$
\Gamma \mapsto \delta(\Gamma)+\Delta(\Gamma)+\sum_{x \in V(\Gamma)} h(x) e_{x}(\Gamma)+\sum_{x \in V(\Gamma)} a_{x}(\Gamma)=\Delta(\Gamma)+\sum_{x \in V(\Gamma)} \frac{1}{2} s_{x}(\Gamma) .
$$

The first sum corresponds to connecting a hair to one $\sigma_{1}$ in $\alpha$, and the second sum corresponds to connecting a hair from one $\sigma_{1}$ to the main part.

Lemma B.2 shows that this complex is acyclic. So, only the class without the main part, $[\alpha]$ survives. That was to be demonstrated. 


\section{Deleting vertices in hairy graphs}

In this section we introduce a few maps that manipulate hairs in hairy graphs with low number of hairs, and also delete some vertices. The simplest example is $D^{(1)}$ defined on graphs with one hair that deletes the hairy vertex in a similar way as deleting a vertex $D$. We prove a list of lemmas and propositions needed in final sections 6 and 7 .

\subsection{One hair}

Let $D^{(1)}: \mathrm{H}^{1} \mathrm{fHGC}_{-1, n} \rightarrow \mathrm{H}^{0} \mathrm{fHGC}_{-1, n}$ be defined on a graph $\Gamma$ as

$$
D^{(1)}(\Gamma)=(-1)^{v(x)} \tilde{D}_{x}
$$

where $x$ is the vertex with the hair, $v(x)$ is its valence and $\tilde{D}_{x}$ deletes vertex $x$ and the hair, and sums over all ways of reconnecting edges that were connected to $x$ to the other vertices, skipping graphs with a tadpole. An example is sketched in Figure 6 .

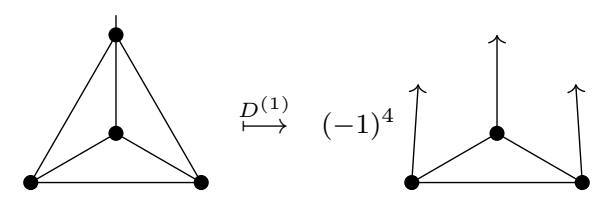

Fig. 6 Example of the action $D^{(1)}$, deleting the hairy vertex. The graph at the right means the sum over all graphs that can be formed by attaching ends of arrows to vertices, skipping graphs with a tadpole.

Also, let the "pushing the hair" $\tilde{D}^{(p u s h)}: \mathrm{H}^{1} \mathrm{fHGC}_{-1, n} \rightarrow \mathrm{H}^{1} \mathrm{fHGC}_{-1, n}$ be defined on a graph $\Gamma$ as

$$
\tilde{D}^{(p u s h)}(\Gamma)=(-1)^{v(x)} \sum_{y} v(x, y) \tilde{D}_{x}^{y}(\Gamma)
$$

where $x$ is again the vertex with the hair, $y$ runs through all vertices of $\Gamma, v(x, y)$ is the number of edges between vertices $x$ and $y$, and whenever $v(x, y)>0 \tilde{D}_{x}^{y}$ deletes vertex $x$, the hair and one edge between $x$ and $y$, sums over all ways of reconnecting the other edges that were connected to $x$ to the other vertices, skipping graphs with a tadpole, and adds a hair on $y$.

For a graph $\Gamma \in \mathrm{H}^{1} \mathrm{fHGC}_{-1, n}^{\geq 2}$ let

$$
D^{(p u s h)}(\Gamma):=\frac{1}{v(x)-1} \tilde{D}^{(p u s h)}(\Gamma),
$$

where $x$ is the hairy vertex. An example is sketched in Figure 7 .

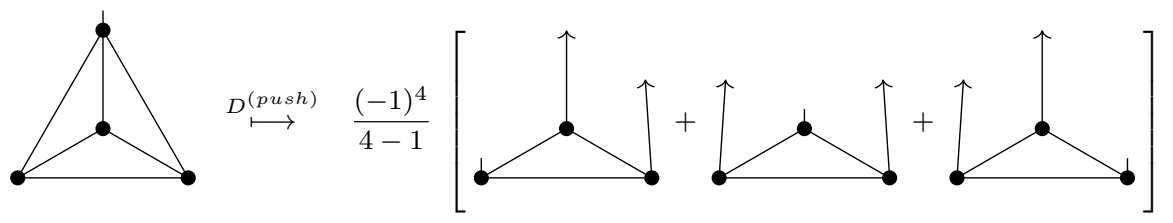

Fig. 7 Example of the action $D^{(\text {push })}$, pushing the hair. The graphs at the right mean the sum over all graphs that can be formed by attaching ends of arrows to vertices, without making a tadpole.

Lemma 5.1. On $\mathrm{H}^{1} \mathrm{fHGC}_{-1, n}^{\geq 2}$ it holds that

$$
D^{(1)}=\Delta D^{(\text {push })} .
$$


Proof. As defined $D^{(1)}$ deletes the hairy vertex $x$ and reconnects its edges to all other edges in all possible ways. $D^{\text {(push) }}$ "saves" one of those edges by transforming it into a hair, and does the same reconnection with the other edges. The operation $\Delta$ after that connects the hair ("saved edge") to other edges in all possible ways, so the result is the same as the one of $D^{(1)}$. We can save $v(x)-1$ different edges (one valence comes from the hair), so $\Delta D^{(\text {push })}$ does the same thing $v(x)-1$ times. It is cancelled by the factor $\frac{1}{v(x)-1}$ from 5.3 . Another factor $(-1)^{v(x)}$ is the same in both definitions. That concludes the proof.

The following lemma would not work on $\mathrm{fHGC}_{-1, n}^{\geq 2}$. But we need such a claim in the proof of Proposition 6.9. This was the very reason for strengthening the constraint $\geq 2$ to $\natural$, see Definition 4.6 .

Lemma 5.2. On $\mathrm{H}^{1} \mathrm{fHGC}_{-1,0}^{\natural}$ it holds that

$$
D^{(1)} D^{(\text {push })}=0
$$

Proof. Recall that in $\mathrm{fHGC}_{-1,0}$ there are no multiple edges. Let $\Gamma \in \mathrm{H}^{1} \mathrm{fHGC}_{-1, n}^{\natural}$ be a graph, and $x$ the hairy vertex in $\Gamma$. One term in $D^{(1)} D^{(p u s h)}(\Gamma)$ will delete the hair, the vertex $x$, one of its neighbours $y$ and edge between them, and reconnect all edges that were connected to $x$ and $y$ elsewhere.

Recall that the constraint $\downarrow$ means that all vertices are at least 2 -valent, and all hairy vertices are at least 3-valent. Therefore $x$ has at least two edges adjacent to it. Chose an edge $e$ at $x$ that did not go towards $y$. That edge can go directly to its final destination, or first to $y$ and then to final destination. Those two terms will cancel, implying the result.

Proposition 5.3. In $\mathrm{H}^{1} \mathrm{fHGC}_{-1, n}$ it holds that

$$
\delta D^{(1)}-D^{(1)} \delta=\Delta .
$$

Proof. Let $\Gamma \in \mathrm{H}^{1} \mathrm{fHGC}_{-1, n}$ be a graph and let $x$ be the hairy vertex in $\Gamma$. Then

$$
\begin{aligned}
& \delta D^{(1)}(\Gamma)-D^{(1)} \delta(\Gamma)= \\
= & \sum_{\substack{y \\
y \neq x}}\left(\frac{1}{2} s_{y} D_{x}(\Gamma)-a_{y} D_{x}(\Gamma)\right)-D_{x} \sum_{\substack{y \\
y \neq x}}\left(\frac{1}{2} s_{y}(\Gamma)-a_{y}(\Gamma)\right)-D_{x}\left(s_{x}^{\prime}(\Gamma)-a_{x}(\Gamma)\right)+D_{z} e_{x}(\Gamma)= \\
= & \frac{1}{2} \sum_{\substack{y \\
x \neq y}} s_{y} D_{x}(\Gamma)-\sum_{\substack{y \\
x \neq y}} a_{y} D_{x}(\Gamma)-\frac{1}{2} \sum_{\substack{y \\
x \neq y}} D_{x} s_{y}(\Gamma)+\sum_{\substack{y \\
x \neq y}} D_{x} a_{y}(\Gamma)-D_{z} s_{x}^{\prime}(\Gamma)+D_{x} a_{x}(\Gamma)+D_{z} e_{x}(\Gamma),
\end{aligned}
$$

where $y$ runs through $V(\Gamma), z$ is the name of the newly added vertex and $s_{x}^{\prime}$ is the part of $s_{x}$ which moves the hair to the new vertex $z$. It holds that $s_{x}=2 s_{x}^{\prime}$.

Using the same arguments as in the proof of Proposition 3.3 it follows that:

$$
\begin{gathered}
s_{y} D_{x}(\Gamma)=D_{x} s_{y}(\Gamma), \\
D_{z} s_{x}^{\prime}(\Gamma)=-\sum_{\substack{y \\
y \neq x}} a_{y} D_{x}(\Gamma), \\
D_{x} a_{x}(\Gamma)=-\sum_{\substack{y \\
y \neq x}} D_{x} a_{y}(\Gamma) .
\end{gathered}
$$

It clearly holds that

$$
D_{z} e_{x}(\Gamma)=\Delta(\Gamma)
$$

so the claimed formula follows.

Proposition 5.4. In $\mathrm{H}^{2} \mathrm{fHGC}_{-1,1}$ it holds that

$$
D^{(1)} \Delta=0 \text {. }
$$


Proof. Let $\Gamma \in \mathrm{H}^{2} \mathrm{fHGCc}_{-1,1}$ be a graph. Recall that for symmetry reasons for odd $n=1$ both hairs can not be on the same vertex. Let them be on vertices $x$ and $y$. The left-hand side is

$$
D^{(1)} \Delta(\Gamma)=D^{(1)}\left(\Delta_{x}(\Gamma)+\Delta_{y}(\Gamma)\right)=D_{y} \Delta_{x}(\Gamma)+D_{x} \Delta_{y}(\Gamma) .
$$

In the first term $\Delta_{x}$ first deletes the hair on $x$ and connects an edge $f$ from $x$ to all other vertices. Then $D_{y}$ deletes a vertex $y$ and reconnects all its edges to other vertices in all possible ways. If $f$ has been connected to $y$, it is also reconnected to all other vertices, what is exactly the same term as if $f$ has been connected at first to its final destination, but with the opposite sign. So, the terms cancel and $D_{y} \Delta_{x}(\Gamma)=0$. The same argument leads to $D_{x} \Delta_{y}(\Gamma)=0$, so the formula holds.

\subsection{Two hairs}

A special care has to be taken for even $n$, i.e. in the complex fHGC $_{-1,0}$ because here one vertex can have multiple hairs. Results of this subsection are therefore used only in Section 7, particularly for Lemma 7.14 used in the proof of Proposition 7.11.

We define $D^{(2-1)}: \mathrm{H}^{2} \mathrm{fHGC}_{-1,0} \rightarrow \mathrm{H}^{1} \mathrm{fHGC}_{-1,0}$. Let $\Gamma \in \mathrm{H}^{2} \mathrm{fHGC}_{-1,0}$ be a graph whose both hairs are on the 3 -valent vertex $y$, and let $x$ be another end of the only edge at $y$, like in the picture:

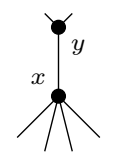

We call this structure a flower on the vertex $y$ with the root $x$. Then $D^{(2-1)}(\Gamma)$ is the graph obtained from gamma by deleting the flower, i.e. both hairs, vertex $y$ and the edge between $x$ and $y$, and puts a hair on $x$. For the matter of sign we consider the edge in the flower to be the last one. If $\Gamma$ is not of that type, $D^{(2-1)}(\Gamma)=0$. Then $D^{(2)}: \mathrm{H}^{2} \mathrm{fHGC}_{-1,0} \rightarrow \mathrm{H}^{0} \mathrm{fHGC}_{-1,0}$ is defined as

$$
D^{(2)}(\Gamma):=D^{(1)} D^{(2-1)}(\Gamma) \text {. }
$$

An example is sketched in Figure 8 .

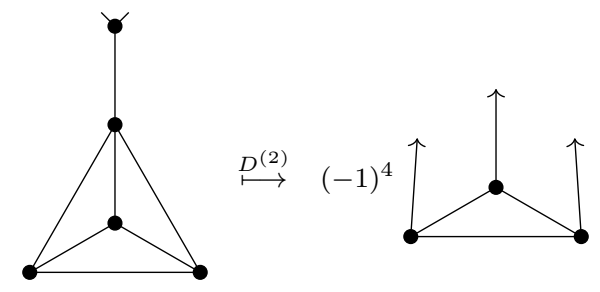

Fig. 8 Example of the action $D^{(2)}$, deleting the flower. The graph at the right means the sum over all graphs that can be formed by attaching ends of arrows to vertices, without making a tadpole.

Proposition 5.5. In the connected part $\mathrm{H}^{2} \mathrm{fHGCc}_{-1,0}$ it holds that

$$
D^{(1)} \Delta=2 \nabla\left(\delta D^{(2)}+D^{(2)} \delta\right) .
$$

Proof. We do the proof of the first claim for four different cases of $\Gamma \in \mathrm{H}^{2} \mathrm{fHGCc}_{-1,0}$ :

1. $\Gamma$ has hairs on two different vertices, $x$ and $y$. By the definition $\delta D^{(2)}(\Gamma)=\delta(0)=0$. Differential $\delta$ can not move hairs to the same vertex, so it is also $D^{(2)} \delta(\Gamma)=0$. The left-hand side is zero because of the same argument as in the proof of Proposition 5.4. hence the formula holds.

2. $\Gamma$ has both hairs on the same vertex that is 2-valent. Because $\Gamma$ is connected it must be $\Gamma=\sigma_{2}=\bullet$, and the formula is easily checked. 
3. $\Gamma$ has both hairs on the same vertex $y$ that is 3 -valent. Let $x$ be another end of the only edge at $y$, i.e. there is a flower on $y$ with the root $x$.

$$
\begin{aligned}
\delta D^{(2)}(\Gamma)+D^{(2)} \delta(\Gamma) & =\sum_{\substack{w \\
w \neq x, y}}\left(\frac{1}{2} s_{w} D^{(2)}(\Gamma)-a_{w} D^{(2)}(\Gamma)\right) \\
& +D^{(2)} \sum_{\substack{w \\
w \neq x, y}}\left(\frac{1}{2} s_{w}(\Gamma)-a_{w}(\Gamma)\right)+D^{(2)}\left(\frac{1}{2} s_{x}(\Gamma)-a_{x}(\Gamma)\right)+D^{(2)} f_{y}(\Gamma)
\end{aligned}
$$

where $s_{y}^{\prime}$ is the term of $s_{y}$ that splits $y$ to $y$ and $z$ where both hairs stay at $y$ and the edge goes to $z$. Other terms of $s_{y}$ cancel out with $a_{y}$ and $h(y) e_{y}$. Using similar arguments as in the proof of Proposition 3.3 one easily checks that

$$
\begin{gathered}
s_{w} D^{(2)}(\Gamma)=-D^{(2)} s_{w}(\Gamma), \\
\frac{1}{2} D^{(2)} s_{x}(\Gamma)=\sum_{\substack{w \\
w \neq x, y}} a_{w} D^{(2)}(\Gamma), \\
D^{(2)} a_{x}(\Gamma)=-\sum_{\substack{w \\
w \neq x, y}} D^{(2)} a_{w}(\Gamma), \\
2 \nabla D^{(2)} s_{y}^{\prime}(\Gamma)=D^{(1)} \Delta(\Gamma),
\end{gathered}
$$

so the formula follows.

4. $\Gamma$ has both hairs on the same vertex $x$ that is more than 3 -valent. Then by the definition $\delta D^{(2)}(\Gamma)=$ $\delta(0)=0$ and the only term that remains from $D^{(2)} \delta(\Gamma)$ is $D^{(2)} s_{x}^{\prime}(\Gamma)$, where $s_{x}^{\prime}$ is the term of $s_{x}$ that splits $x$ to $x$ and $y$ where both hairs stay at $x$ and all edges go to $y$. It still holds $2 \nabla D^{(2)} s_{y}^{\prime}(\Gamma)=$ $D^{(1)} \Delta(\Gamma)$, so the formula follows.

Note that the previous proposition fails for the disconnected graph $\Gamma \cup \sigma_{2} \in \mathrm{H}^{2} \mathrm{fHGC}_{-1,0}$ where $\Gamma \in \mathrm{H}^{0} \mathrm{fHGC}_{-1,0}$.

Proposition 5.6. For the connected part $\mathrm{H}^{3} \mathrm{fHGCc}_{-1,0}$ it holds that

$$
\nabla D^{(2)} \Delta=0
$$

Proof. The only possibility that $D^{(2)} \Delta(\Gamma)$ is not 0 is when $\Delta(\Gamma)$ has a flower, say at a vertex $y$ with the root $x$. If the edge from $x$ to $y$ is created by $\Delta, y$ was disconnected from the rest of the graph in $\Gamma$, what is not possible. So the flower already existed in $\Gamma$. We have 2 cases:

1. The third hair in $\Gamma$ is on the vertex $w \neq x$. Then the part of $\Delta$ that saves the flower deletes the hair on $w$ and makes an edge between $w$ and another vertex $z$ that is not $x$. If $z=x, D^{(2)}$ will move it again to another vertex, cancelling the term where $\Delta$ sent it to its final destination immediately.

2. The third hair in $\Gamma$ is on the vertex $x . \Delta$ deletes it and makes an edge between $x$ and another vertex. $D^{(2)}$ then reconnects the edge from $x$ to another vertex, so the resulting action is adding an edge in all possible ways. Then $\nabla$ adds another edge in all possible ways. Adding one edge on one place and another edge on another place cancels with adding edges in the opposite order.

Note that the previous proposition fails for the disconnected graph $\Gamma \cup \sigma_{3} \in \mathrm{H}^{3} \mathrm{fHGC}_{-1,0}$ where $\Gamma \in \mathrm{H}^{0} \mathrm{fHGC}_{-1,0}$. 


\section{Hairy complex, even edges and odd hairs}

In this section we prove the first part of Theorem 1.1. i.e. that $\mathcal{H}\left(\mathrm{HGC}_{-1,1}, \delta+\Delta\right)=0$. The following diagram describes the way to do that. 'Almost acyclic' means that there are only a few classes of cohomology that are easy to calculate.

The easiest way to show that a complex with a differential $\delta+\Delta$ is acyclic would be to make the spectral sequence in which the first differential is $\Delta$, and to use the fact that the complex with the differential $\Delta$ is acyclic. But neither $\left(\mathrm{H}^{\geq 1} \mathrm{fHGC}_{-1,1}^{\geq 1}, \Delta\right)$ nor $\left(\mathrm{fHGC}_{-1,1}^{\geq 1}, \Delta\right)$ is acyclic, there are classes with 1 or no hairs. For technical reasons we need to disallow $\lambda$ as a connected component and change the constraint $\geq 1$ to $\dagger$. We then change the hairless part in $\left(\mathrm{fHGC}_{-1,1}^{\dagger}, \Delta\right)$ to kill classes with 1 or no hairs and make the new complex, $\left(\mathrm{H}^{\mathrm{b}} \mathrm{fHGC}_{-1,1}^{\dagger}, \Delta\right)$, almost acyclic (Corollary 6.5.

The spectral sequence argument now leads to the conclusion that the complex with the differential $\delta+\Delta$ is almost acyclic (Proposition 6.6). But it is not our intended result, there is a complicated hairless part. To remove it (Proposition 6.8), we need a change of constraint to 4 (at least 2-valent vertices, at least 3 -valent hairy vertices). The standard result that the change of constraint does not change the cohomology of the standard differential $\delta$ (Corollary 4.9), can be used in the spectral sequence with the standard differential being the first one (Proposition 6.8). But the spectral sequence is bounded, and hence converges correctly, only if we change to the bounded complex $\mathrm{H}^{\mathrm{b}} \mathrm{fHGC}_{-1,1}^{\ddagger}$ before that (Proposition 6.7 .

$$
\begin{aligned}
& \sqrt{6.1} 6.2 \\
& \left(\mathrm{fHGC}_{-1,1} \oplus \mathrm{fGC}_{1}^{\geq 1}[-3], \Delta+D^{(1)}\right) \text { is almost acyclic, except for the classes without hairs } \\
& \sqrt{6.3} 6.4 \\
& \left(\mathrm{fHGC}_{-1,1}^{\dagger} \oplus \mathrm{fGC}_{1}^{\dagger}[-3], \Delta+D^{(1)}\right) \text { is almost acyclic, except for the classes without hairs } \\
& \Downarrow 6.5 \\
& \left(\mathrm{H}^{\mathrm{b}} \mathrm{fHGC} \mathrm{C}_{-1,1}^{\dagger}, \Delta+D^{(1)}\right) \text { is almost acyclic } \\
& \sqrt{6.6} \\
& \left(\mathrm{H}^{\mathrm{b}} \mathrm{fHGC}_{-1,1}^{\dagger}, \delta^{\prime}+\Delta+D^{(1)}\right) \text { is almost acyclic } \\
& \Downarrow 6.7
\end{aligned}
$$

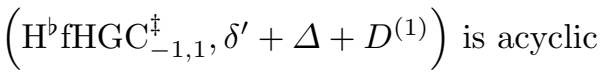

$$
\begin{aligned}
& \Downarrow 6.8 \\
& \left(\mathrm{H}^{\mathrm{b}} \mathrm{fHGC}_{-1,1}^{\natural}, \delta^{\prime}+\Delta+D^{(1)}\right) \text { is acyclic } \\
& \sqrt{6.9} \\
& \left(\mathrm{H}^{\geq 1} \mathrm{fHGC}_{-1,1}^{\natural}, \delta+\Delta\right) \text { is acyclic } \\
& \Downarrow \sqrt{6.10} \\
& \left(\mathrm{H}^{\geq 1} \mathrm{fHGCc}_{-1,1}^{\natural}, \delta+\Delta\right) \text { is acyclic } \\
& \sqrt{6.11} \\
& \left(\mathrm{HGC}_{-1,1}, \delta+\Delta\right) \text { is acyclic }
\end{aligned}
$$

Recall that in the complex $\mathrm{HGC}_{-1,1}$ and all other complexes we are working with in this section, the degree is $d=v+1-h$. 


\subsection{The differential $\Delta$}

In this subsection we want to study the cohomology of $\left(\mathrm{fHGC}_{-1,1}, \Delta\right)$. We will actually study a slightly different complex with an extra term $\mathrm{H}^{0} \mathrm{fHGC}_{-1,1}[-1]$ :

$$
\left(\mathrm{fHGC}_{-1,1} \oplus \mathrm{H}^{0} \mathrm{fHGC}_{-1,1}[-1], \Delta+D^{(1)}\right)
$$

where $\Delta: \mathrm{fHGC}_{-1,1} \rightarrow \mathrm{fHGC}_{-1,1}$ and $D^{(1)}: \mathrm{H}^{1} \mathrm{fHGC}_{-1,1} \rightarrow \mathrm{H}^{0} \mathrm{fHGC}_{-1,1}[-1]$ is deleting the hairy vertex defined in (5.1). Recall that the degree is $d=v+1-h$, so the degree shift [-1] is necessarily to make the differential of the degree +1 . Proposition 5.4 shows that $D^{(1)} \Delta=0$ in fHGC $_{-1,1}$, so the differential indeed squares to 0 . Note that this complex is actually the mapping cone of $D^{(1)}:\left(\mathrm{fHGC}_{-1,1}, \Delta\right) \rightarrow$ $\left(\mathrm{H}^{0} \mathrm{fHGC}_{-1,1}[-1], 0\right)$.

Recall from 2.11 that $\mathrm{H}^{0} \mathrm{fHGC}_{-1,1}[-1]=\mathrm{fGC}_{1}^{\geq 1}[-3]$. For simplicity we use the latter notation.

Similarly to (2.29), our new complex splits as the product of subcomplexes with fixed number of vertices $v$, with the extra term having $v-1$ vertices:

$$
\left(\mathrm{fHGC}_{-1,1} \oplus \mathrm{fGC}_{1}^{\geq 1}[-3], \Delta+D^{(1)}\right)=\prod_{v \in \mathbb{N}}\left(\mathrm{V}^{v} \mathrm{fHGC}_{-1,1} \oplus \mathrm{V}^{v-1} \mathrm{fGC}_{1}^{\geq 1}[-3], \Delta+D^{(1)}\right) .
$$

In each subcomplex, the degree $d=v+1-h$ is up to the shift equal to the negative number of hairs $-h$. We may write it as:

$$
\begin{aligned}
& \begin{array}{lllll}
d= & v-2 & v-1 & v & v+1
\end{array} \\
& \ldots \stackrel{\Delta}{\longrightarrow} \mathrm{H}^{3} \mathrm{~V}^{v} \mathrm{fHGC}_{-1,1} \stackrel{\Delta}{\longrightarrow} \mathrm{H}^{2} \mathrm{~V}^{v} \mathrm{fHGC}_{-1,1} \stackrel{\Delta}{\longrightarrow} \mathrm{H}^{1} \mathrm{~V}^{v} \mathrm{fHGC}_{-1,1} \stackrel{\Delta}{\longrightarrow} \mathrm{H}^{0} \mathrm{~V}^{v} \mathrm{fHGC}_{-1,1} \\
& \vec{D}_{\mathrm{V}^{v-1}} \oplus
\end{aligned}
$$

The case with one vertex is done separately by hand, as follows.

Proposition 6.1. $\mathcal{H}\left(\mathrm{V}^{1} \mathrm{fHGC}_{-1,1}, \Delta\right)$ is one-dimensional, the class being represented by $\sigma_{1}=\bullet$

Proof. In $\mathrm{V}^{1} \mathrm{fHGC}_{-1,1}$ multiple hairs are not possible because of the symmetry reasons. Single vertex without a hair is excluded because of valence condition. Therefore, the whole complex $\mathrm{V}^{1} \mathrm{fHGC}_{-1,1}$ is one dimensional and generated by $\sigma_{1}$. This implies the proposition.

The general proposition is as follows.

Proposition 6.2. $\mathcal{H}^{d}\left(\mathrm{~V}^{v} \mathrm{fHGC}_{-1,1} \oplus \mathrm{V}^{v-1} \mathrm{fGC}_{1}^{\geq 1}[-3], \Delta+D^{(1)}\right)=0$ for all $v \geq 2$ and $d \leq v$.

Note that the proposition does not say anything about the cohomology at degree $d=v+1$, and for $d \geq v+2$ it is trivially 0 .

Proof. $\mathrm{V}^{v-1} \mathrm{fGC}_{1}^{\geq 1}[-3]$ is isomorphic to the subspace of $\mathrm{V}^{v} \mathrm{fGC}_{1}[-2]$ spanned by graphs with an isolated vertex, the isomorphism being adding an isolated vertex $\cup \sigma_{0}$. Since the proposition does not say anything about the cohomology at degree $v+1$ we may safely replace $\mathrm{V}^{v-1} \mathrm{fGC}_{1}^{\geq 1}[-3]$ with the whole $\mathrm{V}^{v} \mathrm{fGC}_{1}[-2]$. The purpose is to make $D^{(1)}$ not changing the number of vertices, so the new $D^{(1)}: \mathrm{V}^{v} \mathrm{H}^{1} \mathrm{fHGC}_{-1,1} \rightarrow$ $\mathrm{V}^{v} \mathrm{fGC}_{1}[-2]$ reconnects all edges from the hairy vertex and deletes it, but restores the vertex without its hair.

Since the differential does not change the number of vertices, we can use Proposition A.1 and work with fixed number of vertices and distinguish them. Let $\bar{V}^{v}:=\overline{\mathrm{V}}^{v} \mathrm{fHGC}-1,1$ and $\bar{W}^{v}:=\overline{\mathrm{V}}^{v} \mathrm{fGC} \mathrm{C}_{1}[-2]$.

We will show the proposition by induction on $v$. In each step we prove that $\mathcal{H}^{v+1-h}\left(\bar{V}^{v} \oplus \bar{W}^{v}, \Delta+D^{(1)}\right)=0$ for every $h \geq 1$, assuming that the same is true for $v-1$ vertices. It is actually enough to use a weaker assumption, without the claim for $h=1$. So in each step we prove that $\mathcal{H}^{v+1-h}\left(\bar{V}^{v} \oplus \bar{W}^{v}, \Delta+D^{(1)}\right)=0$ assuming that for every $h \geq 2 \mathcal{H}^{v-h}\left(\bar{V}^{v-1} \oplus \bar{W}^{v-1}, \Delta+D^{(1)}\right)=0$, or equivalently, since the added term $\bar{W}^{v-1}$ does not affect the cohomology for $h \geq 2$. assuming that for every $h \geq 2 \mathcal{H}^{v-h}\left(\bar{V}^{v-1}, \Delta\right)=0$. 
The assumption is also true for the $v=1$ because the class from Proposition 6.1 has $h=1$. Therefore proving the base of the induction $(v=2)$ is analogous to proving the step.

On $\bar{V}^{v}$ we choose one vertex, say the last one, and set up a spectral sequence on the total valence $s$ of non-chosen vertices. So, an edge between non-chosen vertices counts twice, a hair on non-chosen vertex and an edge between non-chosen vertex and the chosen vertex counts ones, and hair on the chosen vertex does not count. The differential $\Delta$ can not decrease $s$ and splits $\Delta=\Delta_{0}+\Delta_{1}$ where $\Delta_{0}$ is the part that does not change $s . \Delta_{0}$ connects a hair from a non-chosen vertex to the chosen vertex, see an example on Figure 9 and $\Delta_{1}$ connects something to a non-chosen vertex increasing $s$ always by 1 .

To check the correct convergence, let us split the complex as the product of subcomplexes with fixed $a=e+h$, as in 2.29 :

$$
\left(\bar{V}^{v}, \Delta\right)=\prod_{a \in \mathbb{Z}}\left(\mathrm{A}^{a} \bar{V}^{v}, \Delta\right) .
$$

For fixed degree $v+1-h$, i.e. fixed number of hairs $h, s$ can get only finitely possible values, so the spectral sequence converges correctly in each $\left(\mathrm{A}^{a} \bar{V}^{v}, \Delta\right)$, and therefore in the whole $\left(\bar{V}^{v}, \Delta\right)$.

On the first page of the spectral sequence there is the cohomology $\left(\bar{V}^{v}, \Delta_{0}\right)$. Let $\beta: \bar{V}^{v} \rightarrow \bar{V}^{v}$ be the sum over all edges at the chosen vertex of deleting that edge (as heading towards the chosen vertex for the matter of sign) and putting a hair on non-chosen vertex that was connected to that edge, unless it makes the chosen vertex 0 -valent, being forbidden by definition, see an example on Figure 9 .

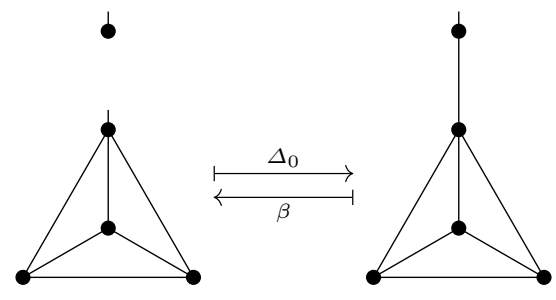

Fig. 9 Example of the actions $\Delta_{0}$ and $\beta$, the chosen vertex is one on the top. It there are more hairs on non-chosen vertices on the left-hand-side, or more edges from the chosen vertex on the right-hand-side, the action is the sum of the actions on all of them.

If the chosen vertex is not hairless or not 1-valent, it is clear that $\Delta_{0} \beta+\beta \Delta_{0}=C$ Id where $C$ is the number of edges at the chosen vertex plus the number of hairs on non-chosen vertices. So all classes of $\mathcal{H}\left(\bar{V}^{v}, \Delta_{0}\right)$ must be represented by graphs whose chosen vertex is isolated with a hair and there are no other hairs $(C=0$ in that case), or the chosen vertex is 1 -valent without a hair ( $\beta$ cannot disconnect the last edge leaving the chosen vertex 0 -valent). Classes that fulfil the former condition we call classes of the first type, and classes that fulfil the latter condition we call classes of the second type.

In what follows, by gluing a 1-vertex graph $\cup \sigma_{0}$ or $\cup \sigma_{1}$ in complexes $\bar{V}^{v}$ with distinguishable vertices we mean adding a new vertex with the highest number, so that it becomes chosen.

Every graph of the form $\Gamma \cup \sigma_{1}, \Gamma \in \mathrm{H}^{0} \bar{V}^{v-1}$, clearly represents a cohomology class of the first type. Graphs whose chosen vertex is 1-valent without a hair would not form classes of the second type if the vertex would be allowed to be 0 -valent. Therefore, cutting that possibility implies that classes of the second type are represented by graphs of the form

$$
\Delta_{0}\left(\Gamma \cup \sigma_{0}\right)=: c(\Gamma)
$$

for $\Gamma \in \mathrm{H}^{\geq 1} \bar{V}^{v-1}$, c.f. the analogous definition for indistinguishable vertices 2.21). All classes are sketched in Figure 10 . 


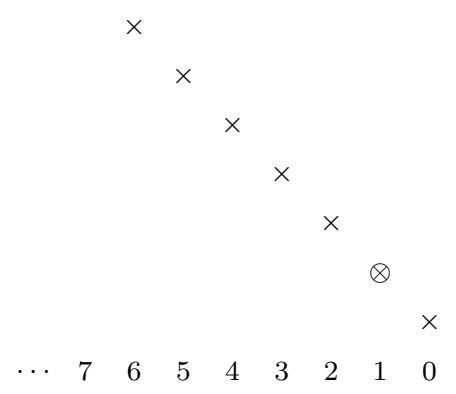

Fig. 10 Classes on the first page of the spectral sequence of $\mathrm{A}^{a} \bar{V}^{v}$ for fixed $a=e+h$. The numbers at the bottom are the numbers of hairs $h$, while the degree is $d=v+1-h$. On the vertical axis is the total valence of non-chosen vertices $s$, the number on which we set up the spectral sequence, starting at $2 a-1$ for $h=0$. Classes of the second type are labelled by $\times$ and the position where there are both classes is labelled by $\otimes$.

Let $\left(\mathrm{C}, \Delta_{1}\right)$ be the complex spanned by second-type classes of the second page of the spectral sequence. It is easily seen that $c:\left(\mathrm{H}^{\geq 1} \bar{V}^{v-1}, \Delta\right) \rightarrow\left(\mathrm{C}, \Delta_{1}\right)$ is an isomorphism of degree 2 , so we can replace $\left(\mathrm{C}, \Delta_{1}\right)$ with $\left(\mathrm{H}^{\geq 1} \bar{V}^{v-1}[-2], \Delta\right)$.

The whole complex on the second page, including first-type classes, is depicted in Figure 11. The first row (only one term in degree $d=v$ ) represents the first-type classes $\Gamma \cup \sigma_{1}$ for $\Gamma \in \mathrm{H}^{0} \bar{V}^{v-1}$. The second row represents the second type classes as discussed above. There is a map that maps first-type class $\Gamma \cup \sigma_{1}$ to the second-type class $\Delta_{1}\left(\Gamma \cup \sigma_{1}\right)$ that is obtained from $\Gamma$ by adding an antenna in all possible ways. The isomorphic element in $\mathrm{H}^{1} \bar{V}^{v-1}[-2]$ is $\chi^{1}(\Gamma)$, where $\chi^{1}$ adds a hair, c.f. (2.19).

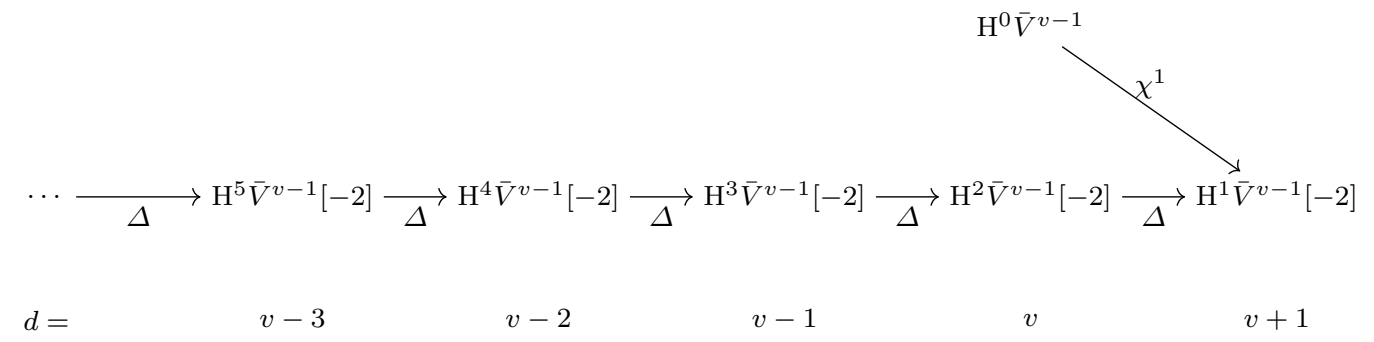

Fig. 11 The complex on the second page of the spectral sequence.

We want to study the cohomology of the complex in the figure for $d \leq v$. Recall that we are not interested in the cohomology at degree $d=v+1$. Here we set up the 2-row spectral sequence as in the figure, such that on the first page there is a complex with horizontal differentials, i.e. $\left(\mathrm{H}^{0} \bar{V}^{v-1}, 0\right)$ and $\left(\bar{V}^{v-1}[-2], \Delta\right)$.

By the induction hypothesis, the cohomology of the second row $\mathcal{H}^{d}\left(\bar{V}^{v-1}[-2], \Delta\right)$ is zero for $h \geq 2$, i.e. in for degrees $d \leq v$ after our degree shift. Let us chose $\Gamma \in \mathrm{H}^{0} \bar{V}^{v-1}$ in the upper row. We do not know whether the $\Gamma$ is cancelled with something in the second row at the degree $d=v+1$. If it is, $\Gamma$ does not for a class in $\mathcal{H}^{d}\left(\bar{V}^{v}, \Delta\right)$.

If it is not, there is a class in degree $d=v$ in the complex from Figure 11, i.e. on the second page of the original spectral sequence represented by $\Gamma+\Gamma_{2}(\Gamma)$ for $\Gamma_{2}(\Gamma) \in \mathrm{H}^{2} V^{v-1}\left(\Gamma_{2}\right.$ depends on $\Gamma)$, where $\chi^{1}(\Gamma)+\Delta\left(\Gamma_{2}(\Gamma)\right)=0$. Back in the starting complex $\left(\bar{V}^{v}, \Delta\right)$ the isomorphic element is $\Gamma \cup \sigma_{1}+\Delta_{0}\left(\Gamma_{2}(\Gamma) \cup \sigma_{0}\right)$ from the second page. It is clearly sent to 0 by the whole $\Delta$, so it represents a class of the starting complex $\bar{V}^{v}$, in the degree $d=v$.

Now we come back to the whole complex $\left(\bar{V}^{v} \oplus \bar{W}^{v}, \Delta+D^{(1)}\right)$. Let us set up a spectral sequence of three rows: $\bar{V}^{v}$, the part of $\bar{W}^{v}$ where the chosen vertex is isolated, and the rest of $\bar{W}^{v}$ (see Figure 12 ). The spectral sequence clearly converges correctly. On the first page in the first row in degree $d=v(h=1)$ we may have classes represented by $\Gamma \cup \sigma_{1}+\Delta_{0}\left(\Gamma_{2}(\Gamma) \cup \sigma_{0}\right)$ for some $\Gamma \in \mathrm{H}^{0} \bar{V}^{v-1}$ and $\Gamma_{2}(\Gamma) \in \mathrm{H}^{2} \bar{V}^{v-1}$, as shown above. In the degree $d=v+1(h=0)$ of the first page we are not interested. In the other rows in the degree $d=v+1(h=0)$ there is still the whole space, particularly in the second row there is $\Gamma \cup \sigma_{0}$ for every $\Gamma \in \mathrm{H}^{0} \bar{V}^{v-1}$. 


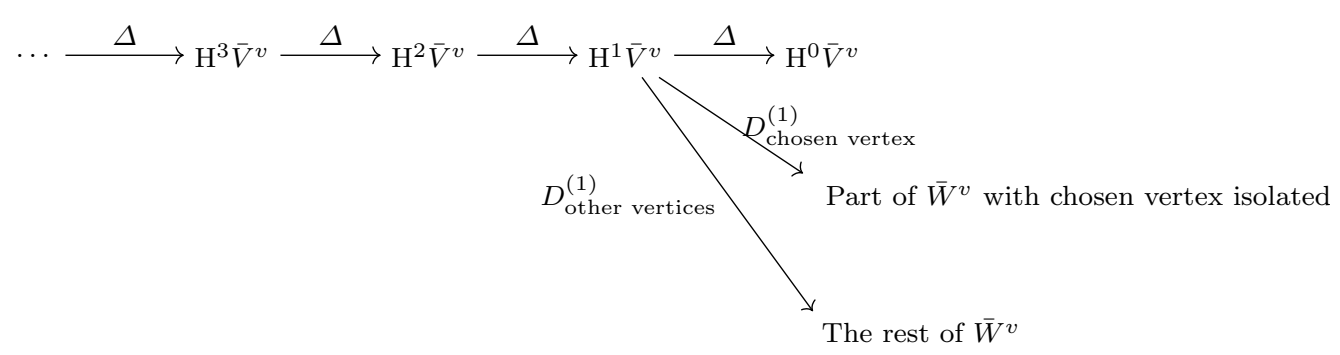

Fig. 12 Complex $\left(\bar{V}^{v}+\bar{W}^{v}, \Delta+D^{(1)}\right)$ split into 3-row spectral sequence.

On the second page $\Gamma \cup \sigma_{1}+\Delta_{0}\left(\Gamma_{2}(\Gamma) \cup \sigma_{0}\right)$ is mapped by part of $D^{(1)}$ to the part of $\bar{W}^{v}$ where the chosen vertex is isolated, i.e. chosen vertex has been deleted (and restored). The only part of $\Gamma \cup \sigma_{1}+$ $\Delta\left(\Gamma_{2}(\Gamma) \cup \sigma_{0}\right)$ that has a hair on the chosen vertex is $\Gamma \cup \sigma_{1}$, so the differential is actually $\Gamma \cup \sigma_{1}+$ $\Delta\left(\Gamma_{2}(\Gamma) \cup \sigma_{0}\right) \mapsto \Gamma \cup \sigma_{0}$. It is clearly an injection, making the cohomology in the degree $d=v$ acyclic. That was to be demonstrated.

\subsection{Removing $\lambda$}

In this subsection we transform the result to the complex with the constraint $\dagger$ that does not have $\lambda=\bullet \bullet$ as a connected component. Recall that it does not have $\sigma_{0}=\bullet$ as a connected component either, because the minimal valence is 1 . Check that $\Delta$ and $D^{(1)}$ can not produce that connected components, so complexes with the the constraint $\dagger$ are closed under their action.

Proposition 6.3. $\mathcal{H}\left(\mathrm{V}^{1} \mathrm{fHGC}_{-1,1}^{\dagger}, \Delta\right)$ is one-dimensional, the class being represented by $\sigma_{1}=\bullet$.

Proof. In the complex of graphs with one vertex there can not be $\lambda$, so $\mathrm{V}^{1} \mathrm{fHGC}_{-1,1}^{\dagger}=\mathrm{V}^{1} \mathrm{fHGC}_{-1,1}$ and the result is the same as in the Proposition 6.1 .

Proposition 6.4. $\mathcal{H}^{d}\left(\mathrm{~V}^{v} \mathrm{fHGC}_{-1,1}^{\dagger} \oplus \mathrm{V}^{v-1} \mathrm{fGC}_{1}^{\dagger}[-3], \Delta+D^{(1)}\right)=0$ for $v>1$ and $d \leq v$.

Proof. We again do the proof by induction on $v$. For $v=2$ there is $\lambda$ only in the hairless part and it represents a cohomology class, so in degrees we are considering it does not change the result of Proposition 6.2 .

Let us pick $v>2$ and assume that the proposition holds for every number of vertices smaller than $v$.

On $\left(\mathrm{V}^{v} \mathrm{fHGC}_{-1,1} \oplus \mathrm{V}^{v-1} \mathrm{fGC}_{1}^{\geq 1}[-3], \Delta+D^{(1)}\right)$ we set up a spectral sequence on the number of $\lambda$-s. The differential can not increase that number, and it is bounded, so the spectral sequence converges correctly. The lowest row on the first page is our intended complex $\left(\mathrm{V}^{v} \mathrm{fHGC}_{-1,1}^{\dagger} \oplus \mathrm{V}^{v-1} \mathrm{fGC}_{1}^{\dagger}[-3], \Delta+D^{(1)}\right)$.

The first differential in the other rows does not effect any $\lambda$, so it is the same as the complex without them, but now with fewer vertices (by 2, 4, etc.), with a degree shift. All of them are acyclic by the assumption of induction in degrees that correspond to more than one hair $(d \leq v-1)$. Therefore, if there is a class with a hair $(d \leq v)$ in the last row, it can not be cancelled by anything, contradicting the result that the whole complex is acyclic in that degrees (Proposition 6.2).

\subsection{Reducing the complex}

To simplify the result we define another complex

$$
\mathrm{H}^{b} \mathrm{~V}^{v} \mathrm{fHGC}_{-1,1}^{\dagger}:=\mathrm{H}^{\geq 1} \mathrm{~V}^{v} \mathrm{fHGC}_{-1,1}^{\dagger} \oplus\left(\Delta+D^{(1)}\right)\left(\mathrm{H}^{1} \mathrm{~V}^{v} \mathrm{fHGC}_{-1,1}^{\dagger}\right) .
$$


We have changed the term with the highest degree $\mathrm{H}^{0} \mathrm{~V}^{v} \mathrm{fHGC}_{-1,1}^{\dagger} \oplus \mathrm{V}^{v-1} \mathrm{fGC}_{1}^{\dagger}[-3]$ with its subspace $\left(\Delta+D^{(1)}\right)\left(\mathrm{H}^{1} \mathrm{~V}^{v} \mathrm{fHGC}_{-1,1}^{\dagger}\right)$, the image of the differential, to ensure the acyclicity at that degree. The whole complex, including all numbers of vertices, is

$$
\mathrm{H}^{\mathrm{f} f H G C}{ }_{-1,1}^{\dagger}:=\prod_{v \in \mathbb{N}} \mathrm{H}^{\mathrm{b}} \mathrm{V}^{v} \mathrm{fHGC}_{-1,1}^{\dagger}=\mathrm{H}^{\geq 1} \mathrm{fHGC}_{-1,1}^{\dagger} \oplus \mathrm{HL}_{1}
$$

where

is the hairless part.

$$
\mathrm{HL}_{1}:=\left(\Delta+D^{(1)}\right)\left(\mathrm{H}^{1} \mathrm{fHGC}_{-1,1}^{\dagger}\right) \subset \mathrm{H}^{0} \mathrm{fHGC}_{-1,1}^{\dagger} \oplus \mathrm{fGC}_{1}^{\dagger}[-3]
$$

Corollary 6.5. $\mathcal{H}\left(\mathrm{H}^{\mathrm{b}} \mathrm{fHGC}_{-1,1}^{\dagger}, \Delta+D^{(1)}\right)$ is one-dimensional, the class being represented by $\sigma_{1}$.

6.4 The differential $\delta+\Delta$

On fHGC ${ }_{-1,1}^{\dagger}$ there is the standard differential $\delta$. We extend it to $\delta^{\prime}: \mathrm{fHGC}_{-1,1}^{\dagger} \oplus \mathrm{fGC}_{1}^{\geq 1}[-3] \rightarrow$ $\mathrm{fHGC}_{-1,1}^{\dagger} \oplus \mathrm{fGC}_{1}^{\geq 1}[-3]$ as follows

$$
\delta^{\prime}(\Gamma, \gamma)=\left(\delta(\Gamma), \mathrm{H}^{0} \Gamma-\delta(\gamma)\right),
$$

where $\mathrm{H}^{0} \Gamma$ is part of $\Gamma$ without hairs. It clearly squares to 0 and has degree 1 , so it is a differential.

Differentials $\delta$ and $\Delta$ anti-commute (Lemma 2.4), so $\left(\mathrm{H}^{\geq 1} \mathrm{fHGC}_{-1,1}^{\dagger}, \delta+\Delta\right)$ is a complex. Proposition 5.3 implies that

$$
\begin{array}{r}
\left(\Delta+D^{(1)}\right) \delta^{\prime}(\Gamma, \gamma)+\delta^{\prime}\left(\Delta+D^{(1)}\right)(\Gamma, \gamma)=\left(\Delta+D^{(1)}\right)\left(\delta(\Gamma), \mathrm{H}^{0} \Gamma-\delta(\gamma)\right)+\delta^{\prime}\left(\Delta(\Gamma), D^{(1)} \mathrm{H}^{1} \Gamma\right)= \\
=\left(\Delta \delta(\Gamma), D^{(1)} \mathrm{H}^{1} \delta(\Gamma)\right)+\left(\delta \Delta(\Gamma), \mathrm{H}^{0} \Delta(\Gamma)-\delta D^{(1)} \mathrm{H}^{1} \Gamma\right)=(0,0),
\end{array}
$$

i.e. $\delta^{\prime}$ and $\Delta+D^{(1)}$ anti-commute and $\left(\mathrm{H}^{\geq 1} \mathrm{fHGC}_{-1,1}^{\dagger} \oplus \mathrm{fGC}_{1}^{\dagger}[-3], \delta^{\prime}+\Delta+D^{(1)}\right)$ is also a complex. Because of the same reason the restriction $\delta^{\prime}: \mathrm{H}^{\mathrm{b} f H G C} \mathrm{fH}_{-1,1}^{\dagger} \rightarrow \mathrm{H}^{\mathrm{b}} \mathrm{fHGC}_{-1,1}^{\dagger}$ is well defined, so

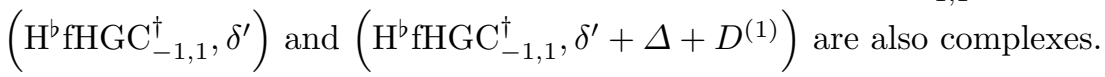

Proposition 6.6. $\mathcal{H}\left(\mathrm{H}^{\mathrm{b}} \mathrm{fHGC} \mathrm{C}_{-1,1}^{\dagger}, \delta^{\prime}+\Delta+D^{(1)}\right)$ is one-dimensional, the class being represented by $\alpha=\sum_{n \geq 1} \frac{1}{n !} \sigma_{1}^{\cup n}$.

Proof. We set up a spectral sequence on $v$ from the splitting 6.2), such that the first differential is $\Delta+D^{(1)}$. By Corollary 6.5 on the first page survives only $\sigma_{1}$.

We can split the complex $\left(\mathrm{fHGC}_{-1,1}^{\dagger} \oplus \mathrm{fGC}_{1}^{\dagger}[-3], \delta^{\prime}+\Delta+D^{(1)}\right)$ as the product of subcomplexes with fixed $f=e+h-v$ :

$$
\left(\mathrm{fHGC}_{-1,1}^{\dagger} \oplus \mathrm{fGC}_{1}^{\dagger}[-3], \delta^{\prime}+\Delta+D^{(1)}\right)=\prod_{f \in \mathbb{Z}}\left(\mathrm{F}^{f} \mathrm{fHGC}_{-1,1}^{\dagger} \oplus \mathrm{F}^{f} \mathrm{fGC}_{1}^{\dagger}[-3], \delta^{\prime}+\Delta+D^{(1)}\right) .
$$

The same splitting can be done for the subcomplex $\mathrm{H}^{\mathrm{b}} \mathrm{fHGC}_{-1,1}^{\dagger}$ :

$$
\left(\mathrm{H}^{\mathrm{f} f H G C} \mathrm{CH}_{-1,1}^{\dagger}, \delta^{\prime}+\Delta+D^{(1)}\right)=\prod_{f \in \mathbb{Z}}\left(\mathrm{F}^{f} \mathrm{H}^{\mathrm{f}} \mathrm{fHGC}_{-1,1}^{\dagger}, \delta^{\prime}+\Delta+D^{(1)}\right) .
$$

For fixed $v$ and degree $d=v+1-h$ the numbers of edges $e$ and hairs $h$ are bounded in each $\mathrm{F}^{f} \mathrm{H}^{\mathrm{b}} \mathrm{HHGC}_{-1,1}^{\dagger}$. The standard spectral sequence argument (e.g. [5. Proposition 19]) implies that the spectral sequence converges correctly in each $\left(\mathrm{F}^{f} \mathrm{H}^{\mathrm{f}} \mathrm{fHGC}_{-1,1}^{\dagger}, \delta^{\prime}+\Delta+D^{(1)}\right)$, and therefore in the whole $\left(\mathrm{H}^{\mathrm{b}} \mathrm{fHGC}_{-1,1}^{\dagger}, \delta^{\prime}+\Delta+D^{(1)}\right)$.

The cohomology of $\left(\mathrm{H}^{\mathrm{b}} \mathrm{fHGC}_{-1,1}^{\dagger}, \delta^{\prime}+\Delta+D^{(1)}\right)$ is therefore one-dimensional. One checks that $\alpha$ is mapped to 0 by the whole differential $\delta^{\prime}+\Delta+D^{(1)}$, and since $\sigma_{1}$ is the highest part of $\alpha, \alpha$ represents the class in $\mathcal{H}\left(\mathrm{H}^{\mathrm{b}} \mathrm{fHGC}^{\dagger}, \delta^{\prime}+\Delta+D^{(1)}\right)$. 


\subsection{Bounded complex}

Recall from Definition 4.4 that bounded complex, the one decorated by $\ddagger$, is spanned by graphs that do not have $\lambda=\bullet \bullet, \sigma_{1}=\boldsymbol{\bullet}$ or $\lambda_{2}=\boldsymbol{\bullet} \bullet$ as a connected component. Let

$$
\mathrm{H}^{\mathrm{b}} \mathrm{fHGC}_{-1,1}^{\ddagger}:=\mathrm{H}^{\geq 1} \mathrm{fHGC}_{-1,1}^{\ddagger} \oplus \mathrm{HL}_{1} \subset \mathrm{H}^{\mathrm{b}} \mathrm{fHGC}_{-1,1}^{\dagger},
$$

where $\mathrm{HL}_{1}$ is as in 6.6.

Proposition 6.7. The complex $\left(\mathrm{H}^{\mathrm{b}} \mathrm{fHGC} \mathrm{C}_{-1,1}^{\ddagger}, \delta^{\prime}+\Delta+D^{(1)}\right)$ is acyclic.

Proof. We write

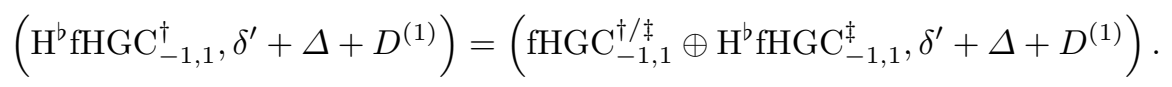

On it we set up a spectral sequence of two obvious rows: fHGC $\mathrm{C}_{-1,1}^{\dagger / \ddagger}$ and $\mathrm{H}^{\mathrm{b}} \mathrm{fHGC} \mathrm{C}_{-1,1}^{\ddagger}$. The spectral sequence clearly converges correctly.

Proposition 4.13 implies that in the first page there is a class $[\alpha]$ in the first row. The class survives all pages because of Proposition 6.6 that says that the whole complex has the class $[\alpha]$. Therefore the second row has to be acyclic. That was to be demonstrated.

6.6 At least 2-valent vertices

Let

$$
\mathrm{H}^{\mathrm{b}} \mathrm{fHGC}_{-1,1}^{\natural}:=\mathrm{H}^{\geq 1} \mathrm{fHGC}_{-1,1}^{\natural} \oplus \mathrm{HL}_{1} \subset \mathrm{H}^{\mathrm{b}} \mathrm{fHGC}_{-1,1}^{\dagger} \text {. }
$$

Recall Definition 4.6 that $\mathrm{HHGC}_{-1,1}^{\natural}$ is the complex spanned by graphs whose vertices are at least 2valent, and hairy vertices are at least 3 -valent.

Proposition 6.8. The complex $\left(\mathrm{H}^{\mathrm{b}} \mathrm{fHGC}{ }_{-1,1}^{\natural}, \delta^{\prime}+\Delta+D^{(1)}\right)$ is acyclic.

Proof. We prove that the inclusion $\left(\mathrm{H}^{\mathrm{b}} \mathrm{fHGC}{ }_{-1,1}^{\natural}, \delta^{\prime}+\Delta+D^{(1)}\right) \hookrightarrow\left(\mathrm{H}^{\mathrm{b}} \mathrm{fHGC}_{-1,1}^{\ddagger}, \delta^{\prime}+\Delta+D^{(1)}\right)$ is a quasi-isomorphism and use Proposition 6.7. We show that the mapping cone is acyclic. On it let us set up a spectral sequence on the number of hairs $h$.

We again split complexes as the product of subcomplexes with fixed $f=e+h-v$ :

$$
\begin{aligned}
& \left(\mathrm{H}^{\mathrm{b} f H G C} \mathrm{CH}_{-1,1}^{\natural}, \delta^{\prime}+\Delta+D^{(1)}\right)=\prod_{f \in \mathbb{Z}}\left(\mathrm{F}^{f} \mathrm{H}^{\mathrm{f}} \mathrm{fHGC}_{-1,1}^{\natural}, \delta^{\prime}+\Delta+D^{(1)}\right), \\
& \left(\mathrm{H}^{\mathrm{b} f H G C}{ }_{-1,1}^{\ddagger}, \delta^{\prime}+\Delta+D^{(1)}\right)=\prod_{f \in \mathbb{Z}}\left(\mathrm{F}^{f} \mathrm{H}^{\mathrm{b} f H G C}{ }_{-1,1}^{\ddagger}, \delta^{\prime}+\Delta+D^{(1)}\right) .
\end{aligned}
$$

For fixed degree $d=v+1-h$ the number of edges $e$ is fixed in each $\mathrm{F}^{f} \mathrm{H}^{\mathrm{f}} \mathrm{fHGC} \mathrm{C}_{-1,1}^{\natural}$ and $\mathrm{F}^{f} \mathrm{H}^{\mathrm{b}} \mathrm{fHGC} \mathrm{C}_{-1,1}^{\ddagger}$. Also, increasing the number of hairs $h$ increases the number of vertices $v$ by the same amount. Since the number of edges $e$ is fixed, for $h$ big enough, there will be an isolated vertex. But that is not possible in either $\mathrm{H}^{\mathrm{b}} \mathrm{fHGC}_{-1,1}^{\natural}$ or $\mathrm{H}^{\mathrm{b}} \mathrm{fHGC} \mathrm{C}_{-1,1}^{\ddagger}$. So, the spectral sequence of the mapping cone of the inclusion $\left(\mathrm{F}^{f} \mathrm{H}^{\mathrm{b}} \mathrm{fHGC}{ }_{-1,1}^{\natural}, \delta^{\prime}+\Delta+D^{(1)}\right) \hookrightarrow\left(\mathrm{F}^{f} \mathrm{H}^{\mathrm{b}} \mathrm{fHGC}_{-1,1}^{\ddagger}, \delta^{\prime}+\Delta+D^{(1)}\right)$ is bounded above and converges correctly for every $f$, and therefore also the spectral sequence of the mapping cone of the whole inclusion $\left(\mathrm{H}^{\mathrm{b}} \mathrm{fHGC}{ }_{-1,1}^{\natural}, \delta^{\prime}+\Delta+D^{(1)}\right) \hookrightarrow\left(\mathrm{H}^{\mathrm{b}} \mathrm{fHGC}_{-1,1}^{\ddagger}, \delta^{\prime}+\Delta+D^{(1)}\right)$ converges correctly. This convergence is the very reason why we introduced the bounded complex.

On the first page of the spectral sequence, for $h=0$ there is a mapping cone of the identity $\left(\mathrm{HL}_{1}, \delta^{\prime}\right) \rightarrow\left(\mathrm{HL}_{1}, \delta^{\prime}\right)$, so it is acyclic. For $h>0$ there is a mapping cone of the inclusion $\left(\mathrm{fHGC}_{-1,1}^{\natural}, \delta\right) \rightarrow$ $\left(\mathrm{fHGC}_{-1,1}^{\ddagger}, \delta\right)$. It is acyclic by Corollary 4.9 . That concludes the proof. 
6.7 Removing the hairless part

Proposition 6.9. The complex $\left(\mathrm{H}^{\geq 1} \mathrm{fHGC}_{-1,1}^{\natural}, \delta+\Delta\right)$ is acyclic.

Proof. On $\left(\mathrm{H}^{\mathrm{b}} \mathrm{fHGC}_{-1,1}^{\natural}, \delta^{\prime}+\Delta+D^{(1)}\right)$ we set up a spectral sequence of two obvious rows: $\mathrm{H}^{\geq 1} \mathrm{fHGC}_{-1,1}^{\natural}$ and $\mathrm{HL}_{1}$. Clearly the spectral sequence converges correctly. Proposition 6.8 implies that the whole complex is acyclic, so all classes of the first page cancel out. We claim that there are no classes on the first page.

Suppose the opposite, that there is a class in the first row represented by $\Gamma \in \mathrm{H}^{\geq 1} \mathrm{fHGC}_{-1,1}^{\natural}$. Let $\Delta=\Delta_{0}+\Delta_{1}$ where $\Delta_{0}: \mathrm{H}^{\geq 1} \mathrm{fHGC}_{-1,1}^{\natural} \rightarrow \mathrm{H}^{\geq 1} \mathrm{fHGC}_{-1,1}^{\natural}$ and $\Delta_{1}: \mathrm{H}^{\geq 1} \mathrm{fHGC}_{-1,1}^{\natural} \rightarrow \mathrm{HL}_{1}$. It holds that $\left(\delta+\Delta_{0}\right)(\Gamma)=0$ and $\left(\Delta_{1}+D^{(1)}\right)(\Gamma)$ represents a class in $\mathrm{HL}_{1}$. We write $\Gamma=\sum_{h \geq 1} \mathrm{H}^{h} \Gamma$ where $\mathrm{H}^{h} \Gamma$ is the part of $\Gamma$ with $h$ hairs. Then it holds that

$$
\Delta\left(\mathrm{H}^{2} \Gamma\right)+\delta\left(\mathrm{H}^{1} \Gamma\right)=0
$$

and $\left(\Delta+D^{(1)}\right)\left(\mathrm{H}^{1} \Gamma\right)$ represents a class in $\mathrm{HL}_{1}$.

Let $\gamma:=D^{(\text {push })}\left(\mathrm{H}^{1} \Gamma\right)$. It is for sure in $\mathrm{H}^{1} \mathrm{fHGC}_{-1,1}^{\dagger}$. Lemma 5.1 implies

$$
\Delta(\gamma)=D^{(1)}\left(\mathrm{H}^{1} \Gamma\right)
$$

and Lemma 5.2 implies

$$
D^{(1)}(\gamma)=0
$$

This relation was the very reason of introducing the constraint $\bullet$. Propositions 5.3 and 5.4 imply that

$$
\Delta\left(\mathrm{H}^{1} \Gamma\right)=\delta D^{(1)}\left(\mathrm{H}^{1} \Gamma\right)-D^{(1)} \delta\left(\mathrm{H}^{1} \Gamma\right)=\delta D^{(1)}\left(\mathrm{H}^{1} \Gamma\right)+D^{(1)} \Delta\left(\mathrm{H}^{2} \Gamma\right)=\delta D^{(1)}\left(\mathrm{H}^{1} \Gamma\right) .
$$

The equalities are diagrammatically expressed in Figure 13.

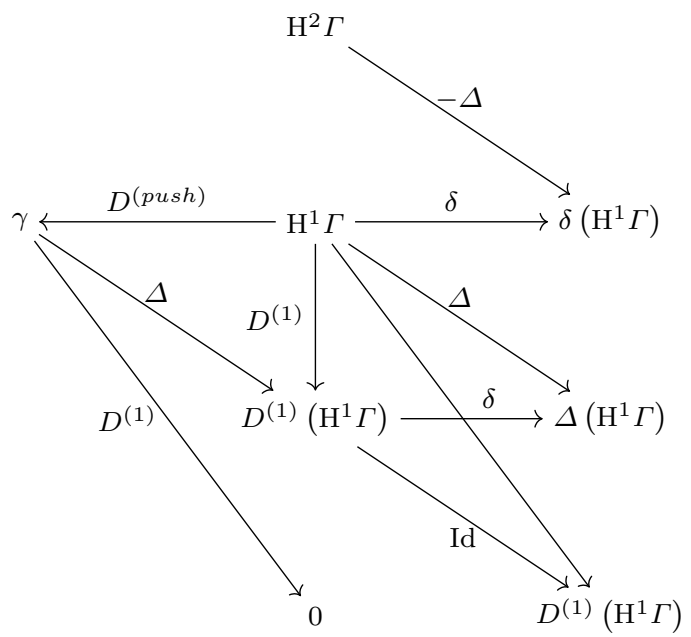

Fig. 13 Maps in $\mathrm{H}^{\geq 1} \mathrm{fHGC}_{-1,1}^{\natural} \oplus \mathrm{H}^{0} \mathrm{fHGC}_{-1,1}^{\dagger} \oplus \mathrm{fGC}_{1}^{\dagger}[-3]$. The rows are, from the bottom up: fGC $\mathrm{fH}_{1}^{\dagger}[-3], \mathrm{H}^{0} \mathrm{fHGC} \mathrm{CH}_{-1,1}^{\dagger}$, $\mathrm{H}^{1} \mathrm{fHGC}_{-1,1}^{\natural}$ and $\mathrm{H}^{2} \mathrm{fHGC}{ }_{-1,1}^{\natural}$.

Note that the complex in the figure is bigger than $\mathrm{H}^{b} \mathrm{fHGC}_{-1,1}^{\natural}$, its hairless part is the whole $\mathrm{H}^{0} \mathrm{fHGC}_{-1,1}^{\dagger} \oplus \mathrm{fGC}_{1}^{\dagger}[-3]$ instead of only $\mathrm{HL}_{1}$. Indeed, both mentioned elements of the hairless part $\left(D^{(1)}\left(\mathrm{H}^{1} \Gamma\right), 0\right)$ and $\left(\delta\left(\mathrm{H}^{1} \Gamma\right), D^{(1)}\left(\mathrm{H}^{1} \Gamma\right)\right)$ are in $\mathrm{HL}_{1}$ because they are images of $\gamma$, respectively $\left(\mathrm{H}^{1} \Gamma\right)$, under the action of $\left(\Delta+D^{(1)}\right)$.

Since $\left(\Delta+D^{(1)}\right)\left(\mathrm{H}^{1} \Gamma\right)=\delta^{\prime}\left(D^{(1)}\left(\mathrm{H}^{1} \Gamma\right), 0\right)$, the former is exact in $\mathrm{HL}_{1}$, contradicting the assumption. 


\subsection{Connected complex}

In this subsection we transform the result to the connected complex.

Proposition 6.10. The complex $\left(\mathrm{H}^{\geq 1} \mathrm{fHGCc}_{-1,1}^{\natural}, \delta+\Delta\right)$ is acyclic.

Proof. The complex splits as:

$$
\left(\mathrm{H}^{\geq 1} \mathrm{fHGCc}_{-1,1}^{\natural}, \delta+\Delta\right)=\prod_{f \in \mathbb{Z}}\left(\mathrm{F}^{f} \mathrm{H}^{\geq 1} \mathrm{fHGCc}_{-1,1}^{\natural}, \delta+\Delta\right),
$$

where $f=e+h-v$. In each of the subcomplexes the degree $d=v+1-h=e+1-f$ is determined by the number of edges $e$. We show that for every $f \in \mathbb{Z} \mathcal{H}^{e+1-f}\left(\mathrm{~F}^{f} \mathrm{H}^{\geq 1} \mathrm{fHGCc}_{-1,1}^{\natural} \delta+\Delta\right)=0$ by the induction on $e$.

For $e=0$ it is clear. Let us suppose that the claim holds for every number of edges less than $e$, i.e. suppose that for every $f$

$$
\mathcal{H}^{d}\left(\mathrm{~F}^{f} \mathrm{H}^{\geq 1} \mathrm{fHGCc}_{-1,1}^{\natural} \delta+\Delta\right)=0
$$

in every degree $d<e+1-f$.

Let us add the hairless part. The assumption implies that the inclusion

$$
\left(\mathrm{F}^{f} \mathrm{H}^{0} \mathrm{fHGCc}{ }_{-1,1}^{\natural}, \delta\right) \hookrightarrow\left(\mathrm{F}^{f} \mathrm{fHGCc}_{-1,1}^{\natural}, \delta+\Delta\right)
$$

is a quasi-isomorphism for every $f$ in every degree $d<e+1-f$.

Let $\Delta_{C}$ be the part of $\Delta$ that does not connect two connected components. Taking symmetric product gives

$$
\begin{aligned}
S^{+}\left(\mathrm{H}^{0} \mathrm{fHGCc}_{-1,1}^{\natural}[-1], \delta+\Delta\right)[1] & =\left(\mathrm{H}^{0} \mathrm{fHGC}_{-1,1}^{\natural}, \delta+\Delta_{C}\right), \\
S^{+}\left(\mathrm{fHGCc}_{-1,1}^{\natural}[-1], \delta+\Delta\right)[1] & =\left(\mathrm{fHGC}_{-1,1}^{\natural}, \delta+\Delta_{C}\right) .
\end{aligned}
$$

Taking cohomology commutes with the symmetric product, so the cohomology of the mapping cone of the inclusion

$$
\left(\mathrm{H}^{0} \mathrm{fHGC}_{-1,1}^{\natural}, \delta\right) \hookrightarrow\left(\mathrm{fHGC}_{-1,1}^{\natural}, \delta+\Delta_{C}\right)
$$

is the symmetric product of the cohomology of the mapping cone of

$$
\left(\mathrm{H}^{0} \mathrm{fHGCc}_{-1,1}^{\natural}, \delta\right) \hookrightarrow\left(\mathrm{fHGCc}_{-1,1}^{\natural}, \delta+\Delta\right)
$$

that split as a direct product of inclusions 6.11). Cohomology of $\mathrm{F}^{f}$ part of 6.14 in degree that corresponds to $e-1$ edges $(d=e-f)$ comes from cohomologies from 6.11) for different $f$-s in degrees that correspond to $\leq e-1$ edges $(d \leq e-f)$. Therefore (6.11) being quasi-isomorphism for every $f$ in every degree $d<e+1-f$ implies that (6.14) is a quasi-isomorphism in degree $d=e-f$.

We need to prove the claim for $e$ edges, i.e. that for every $f$

$$
\mathcal{H}^{e+1-f}\left(\mathrm{~F}^{f} \mathrm{H}^{\geq 1} \mathrm{fHGCc}_{-1,1}^{\natural} \delta+\Delta\right)=0 .
$$

Suppose the opposite, that there is a class represented by $\Gamma \in \mathrm{F}^{f} \mathrm{H}^{\geq 1} \mathrm{fHGCc}_{-1,1}^{\natural}$ of degree $e+1-f$, i.e. it is a sum of graphs with $e$ edges. By Proposition 6.9 there is $\gamma \in \mathrm{H}^{\geq 1} \mathrm{fHGC}_{-1,1}^{\natural}$ of degree $e-f$ (i.e. it is a sum of graphs with $e-1$ edges) such that $\Gamma=(\delta+\Delta)(\gamma)$ in $\mathrm{H}^{\geq 1} \mathrm{fHGCc}_{-1,1}^{\natural}$.

Since in $\mathrm{H}^{\geq 1} \mathrm{~F}^{f}$ fHGC ${ }_{-1,1}^{\natural}$ every connected component has at least one edge, the number of connected components is bounded. We write $\gamma=\sum_{i=1}^{k} \mathrm{C}^{i} \gamma$ where $\mathrm{C}^{i} \gamma$ is the part with $i$ connected components. Choose $\gamma$ such that $k$ is minimal possible. If $k=1$ we are done, so suppose that $k>1$.

It holds that $\mathrm{C}^{k} \gamma \in \mathrm{C}^{k} \mathrm{~F}^{f}$ fHGC $_{-1,1}^{\natural}$ and $\left(\delta+\Delta_{0}\right) \mathrm{C}^{k} \gamma=0 . \mathrm{C}^{k} \gamma$ is of degree $e-f$ (e-1 edges) and inclusion 6.14 is quasi-isomorphism in that degree, so all classes are generated by hairless graphs. Therefore there is a hairless representative of the class $\left[\mathrm{C}^{k} \gamma\right]$, i.e. there is $\gamma^{\prime} \in \mathrm{C}^{k} \mathrm{~F}^{f} \mathrm{fHGC}_{-1,1}^{\natural}$ of degree $e-f-1$ such that $\mathrm{C}^{k} \gamma-\left(\delta+\Delta_{C}\right) \gamma^{\prime}$ is hairless. 
It holds that

$$
(\delta+\Delta)\left(\gamma-(\delta+\Delta) \gamma^{\prime}\right)=\Gamma
$$

$\Delta$ does not act on hairless part, so only $\delta$ acts on the part with the maximal $k$ connected components $\mathrm{C}^{k} \gamma-\left(\delta+\Delta_{0}\right) \gamma^{\prime}$. But $\delta$ can not change the number of connected components, and since right-hand side is connected, it holds that

$$
(\delta+\Delta)\left(\mathrm{C}^{k} \gamma-\left(\delta+\Delta_{0}\right) \gamma^{\prime}\right)=\delta\left(\mathrm{C}^{k} \gamma-\left(\delta+\Delta_{0}\right) \gamma^{\prime}\right)=0
$$

Therefore, that part can be removed and the resulting element is still mapped to $\Gamma$ by $(\delta+\Delta)$. It has less than $k$ connecting components, contradicting the minimality of $k$.

Recall from 2.17) that $\mathrm{HGC}_{-1,1}=\mathrm{H}^{\geq 1} \mathrm{fHGCc}_{-1,1}^{\geq 3}$.

Proposition 6.11 (The first part of Theorem 1.1). The complex $\left(\mathrm{HGC}_{-1,1}, \delta+\Delta\right)$ is acyclic.

Proof. On the mapping cone of the inclusion $\left(\mathrm{HGC}_{-1,1}, \delta+\Delta\right) \rightarrow\left(\mathrm{H}^{\geq 1} \mathrm{fHGCc}_{-1,1}^{\natural}, \delta+\Delta\right)$ we set up a spectral sequence on the number of hairs. The discussion from the proof of Proposition 6.8 implies that the spectral sequence converges correctly.

On the first page there is the mapping cone of the inclusion $\left(\mathrm{HGC}_{-1,1}, \delta\right) \rightarrow\left(\mathrm{H}^{\geq 1} \mathrm{fHGCc}_{-1,1}^{\natural}, \delta\right)$. It is acyclic by Corollary 4.10. This implies that the mapping cone is acyclic, and Proposition 6.10 concludes the proof.

\section{Hairy complex, odd edges and even hairs}

In this section we prove the second part of Theorem 1.1, i.e. that $\mathcal{H}\left(\mathrm{HGC}_{-1,0}, \delta+\Delta\right)$ is one-dimensional, the class being represented by the star $\sigma_{3}$. The proving strategy is very similar to the one in previous section, as shown in the following diagram.

\section{$7.1,7.2,7.3,7.4$}

$\left(\right.$ fHGC $\left._{-1,0}, \Delta\right)$ is almost acyclic, except for the classes without hairs<smiles>C1CCC2(C1)CCC2</smiles>

$\left(\mathrm{fHGC}_{-1,0}, \delta+\Delta\right)$ is almost acyclic, except for classes whose generators have hairless part<smiles>C1=CC2C=CC12</smiles>

$\left(\mathrm{fHGC}_{-1,0}^{\dagger}, \delta+\Delta\right)$ is almost acyclic, except for classes whose generators have hairless part

$$
\begin{aligned}
& \sqrt{7.8} \\
& \left(\mathrm{H}^{\mathrm{b}} \mathrm{fHGC} \mathrm{H}_{-1,0}^{\dagger}, \delta+\Delta\right) \text { is almost acyclic } \\
& \text { 77.9 } \\
& \left(\mathrm{H}^{\mathrm{b}} \mathrm{fHGC} \mathrm{C}_{-1,0}^{\ddagger}, \delta+\Delta\right) \text { is almost acyclic } \\
& \text { 7.11 }
\end{aligned}
$$

$\mathcal{H}\left(\mathrm{H}^{\geq 1} \mathrm{fHGCc}_{-1,0}^{\ddagger}, \delta+\Delta\right)$ is one-dimensional, the class being represented by the star $\sigma_{3}=$<smiles>CC(C)C1CCCC1C</smiles>

$\mathcal{H}\left(\mathrm{HGC}_{-1,0}, \delta+\Delta\right)$ is one-dimensional, the class being represented by the star $\sigma_{3}$

Recall that in the complex $\mathrm{HGC}_{-1,0}$ and all other complexes we are working with in this section, the degree is $d=e+1$. 


\subsection{The differential $\Delta$}

In this subsection we study the cohomology of $\left(\mathrm{fHGC}_{-1,0}, \Delta\right)$. As in 2.29 the complex $\left(\mathrm{fHGC}_{-1,0}, \Delta\right)$ split into the double direct product of complexes, for fixed number of vertices $v$ and for fixed $a=e+h$ :

$$
\left(\mathrm{fHGC}_{-1,0}, \Delta\right)=\prod_{v \in \mathbb{N}} \prod_{a \in \mathbb{Z}}\left(\mathrm{A}^{a} \mathrm{~V}^{v} \mathrm{fHGC}_{-1,0}, \Delta\right)
$$

The cases with one and two vertices are done separately as follows.

Proposition 7.1. $\mathcal{H}\left(\mathrm{A}^{a} \mathrm{~V}^{1} \mathrm{fHGC}_{-1,0}, \Delta\right)$ is 1-dimensional for $a \geq 1$, the class being represented by $\sigma_{a}=$ with a hairs.

Proof. The whole space $\mathrm{A}^{a} \mathrm{~V}^{1} \mathrm{fHGC}_{-1,0}$ is generated only by $\sigma_{a}$, implying the proposition.

Proposition 7.2. $\mathcal{H}\left(\mathrm{A}^{a} \mathrm{~V}^{2} \mathrm{fHGC}_{-1,0}, \Delta\right)$ is zero for even $a \geq 2$ and 1-dimensional for odd $a \geq 1$, the class being represented by $\lambda_{a}=\mathbf{\bullet} \cdot$ with $a-1$ hairs on one vertex.

Proof. Because of the symmetry reasons there are no multiple edges, so there can be either no edge or one edge between the two vertices, i.e. the degree is either 1 on 2 .

In degree 1 graphs are $\sigma_{i} \cup \sigma_{a-i}$ for $i \geq a / 2$ (opposite graph is the same). In degree 2 graphs are $\lambda_{a, i}:=\ldots$ with $i$ and $a-i-1$ hairs for $i \geq(a-1) / 2$.

One easily checks that $\Delta\left(\sigma_{i} \cup \sigma_{a-i}\right)=i \lambda_{a, i-1}+(a-i) \lambda_{a, i}$ for $i>a / 2$ and $\Delta\left(\sigma_{i} \cup \sigma_{i}=2 i \lambda_{a, i}\right.$ for $i=a / 2$. It is now straightforward to check that the cohomology is zero for even $a$ and 1-dimensional for odd $a$, generated by any $\lambda_{a, i}$, in particular by $\lambda_{a, a-1}=\lambda_{a}$.

The following two propositions give result for $v=3$ and the general one, respectively. Let us first define for $a \geq 2$

$$
\rho_{a}:=\sum_{i=1}^{a-1} \frac{(-1)^{i}}{i !(a-1-i) !} \sigma_{i} \cup \lambda_{a-i}=\sum_{i=1}^{a-1} \frac{(-1)^{i}}{i !(a-1-i) !} \underset{a-i-1}{i} \underset{\sim}{i} \in \mathrm{A}^{a} \mathrm{~V}^{3} \mathrm{fHGC}_{-1,0}
$$

Proposition 7.3. $\mathcal{H}\left(\mathrm{A}^{a} \mathrm{~V}^{3} \mathrm{fHGC}_{-1,0}, \Delta\right)$ is acyclic for odd $a \geq 1$ and 1-dimensional for even a $\geq 2$, the class being represented by $\rho_{a}$.

Proposition 7.4. $\mathcal{H}^{a-h+1}\left(\mathrm{~A}^{a} \mathrm{~V}^{v} \mathrm{fHGC}_{-1,0}, \Delta\right)=0$ for $v \geq 4$ and $h \geq 1$.

Note that the general proposition does not say anything about the cohomology at degree $d=a+1$ and for $d>a+1$ it is trivially 0 .

We start proving propositions together, and we will divert at some point. The proof strategy is very similar to its odd analogue Proposition 6.2. The proof is inductive on the number of vertices $v$ and the step uses the spectral sequence on the total valence $s$ of non-chosen vertices. We highlight the key differences for the readers convenience:

- In this case there is no extra non-hairy term.

- Subcomplexes $\left(\mathrm{A}^{a} \mathrm{~V}^{v} \mathrm{fHGC}_{-1,0}, \Delta\right)$ with different $a=e+h$ have sometimes different treatment, so we work with them through the whole proof. That was not necessary in the odd case.

- We again choose one distinguished vertex. In the odd case we simply switched to the complex with distinguishable vertices $\bar{V}^{v} \mathrm{fHGC}_{-1,1}$ and chose one. Here, for inductive argument to work, we must leave other vertices indistinguishable. Therefore we work with the complex $\dot{V}^{v} \mathrm{~A}^{a} \mathrm{fHGC}_{-1,0}$ of graphs with one vertex chosen, and other indistinguishable, c.f. Appendix A

- In this case there can be multiple hairs on one vertex. Therefore, on the first page of the main spectral sequence in the proof, classes of the first type are now of the form $\Gamma \dot{\cup} \sigma_{h}$ for any $h \geq 1$. This introduces the whole new line of classes of the first type, see Figure 14 . To kill them, this time we need to investigate the third page of the spectral sequence too. 
Joint part of the proof of Propositions 7.3 and 7.4. For the matter of shortening the notation let

$$
V_{a}^{v}:=\mathrm{A}^{a} \mathrm{~V}^{v} \mathrm{fHGC}_{-1,0}
$$

On $V_{a}^{v}$ we choose one vertex and get

$$
\dot{V}_{a}^{v}:=\mathrm{A}^{a} \dot{\mathrm{V}}^{v} \mathrm{fHGC}_{-1,0}
$$

the complex with one vertex chosen, while the others are indistinguishable (see appendix A). In what follows we will relate it to the complex of $v-1$ indistinguishable vertices $V_{a}^{v-1}$, i.e. to the original complex with one vertex less. The general structure of the recursive proof is as follows:

- From Proposition 7.2 we know the cohomology of $V_{a}^{2}$. We use it to find cohomology of $\dot{V}_{a}^{3}$, and symmetrizing we get cohomology of $V_{a}^{3}$. This is the content of Proposition 7.3

- We use cohomology of $V_{a}^{3}$ to find cohomology of $\dot{V}_{a}^{4}$. Although it is not zero, after symmetrizing we get that $\mathcal{H}\left(V_{a}^{4}\right)=0$. This is the base of the induction of Proposition 7.4

- We will see that $\mathcal{H}\left(V_{a}^{v-1}\right)=0$ implies $\mathcal{H}\left(\dot{V}_{a}^{v}\right)=0$ and that after symmetrization (Proposition A.2 implies $\mathcal{H}\left(V_{a}^{v}\right)=0$. This is the step of the induction of Proposition 7.4.

Let us set up a spectral sequence on $\left(\dot{V}_{a}^{v}, \Delta\right)$ on the total valence $s$ of non-chosen vertices, including hairs. So, an edge between non-chosen vertices counts twice, a hair on a non-chosen vertex and an edge between a non-chosen vertex and the chosen vertex counts once, and hairs on the chosen vertex do not count. The differential can not decrease $s$ and splits $\Delta=\Delta_{0}+\Delta_{1}$ where $\Delta_{0}$ is the part that does not change $s . \Delta_{0}$ connects a hair from a non-chosen vertex to the chosen vertex and $\Delta_{1}$ connects an edge to a non-chosen vertex, increasing $s$ always by 1 .

For fixed $a=e+h, s$ can have only finitely many possible values, so the spectral sequence is finite and converges correctly.

On the first page of the spectral sequence there is the cohomology of $\left(\dot{V}_{a}^{v}, \Delta_{0}\right)$. Let $\beta: \dot{V}_{a}^{v} \rightarrow \dot{V}_{a}^{v}$ be the sum over all edges at the chosen vertex of deleting that edge (as a last edge in numbering, for the matter of sign) and putting a hair on the non-chosen vertex that was connected to that edge, unless it makes the chosen vertex 0 -valent, being forbidden by definition. Maps $\Delta_{0}$ and $\beta$ are analogous to those in odd case depicted in Figure 9.

If the chosen vertex is not hairless or not 1-valent, it is clear that $\Delta_{0} \beta+\beta \Delta_{0}=C$ Id where $C$ is the number of edges at the chosen vertex plus the number of hairs on non-chosen vertices. So, all classes of $\mathcal{H}\left(\dot{V}_{a}^{v}, \Delta_{0}\right)$ must be represented by graphs whose chosen vertex is isolated with some hairs and there are no other hairs $(C=0$ in that case), or the chosen vertex is 1 -valent without hairs ( $\beta$ cannot disconnect the last edge leaving the chosen vertex 0 -valent). Classes that fulfil the former condition we call classes of the first type, and classes that fulfil the latter condition we call classes of the second type.

Let $\cdot \dot{U} \sigma_{h}: \mathrm{H}^{0} V_{a-h}^{v-1} \rightarrow \dot{V}_{a}^{v}$ be the operation of disjoint union with the star $\sigma_{h}$ that maps $\Gamma$ with distinguishable vertices to $\Gamma \dot{\cup} \sigma_{h}$ with chosen vertex being the one in the star, while the other vertices remain indistinguishable.

Every graph of the form $\Gamma \dot{\cup} \sigma_{h}$, where $\Gamma \in \mathrm{H}^{0} V_{a-h}^{v-1}, h \geq 1$, clearly represents a cohomology class of the first type. Graphs whose chosen vertex is 1-valent without hairs would not form classes of the second type if the vertex would be allowed to be 0 -valent. Therefore, cutting that possibility implies that classes of the second type are represented by graphs of the form

$$
\Delta_{0}\left(\Gamma \dot{\cup} \sigma_{0}\right)=: \dot{c}(\Gamma)
$$

for $\Gamma \in \mathrm{H}^{\geq 1} V_{a}^{v-1}$, c.f. the analogous definition for all indistinguishable vertices 2.21). All classes are sketched in Figure 14 . 


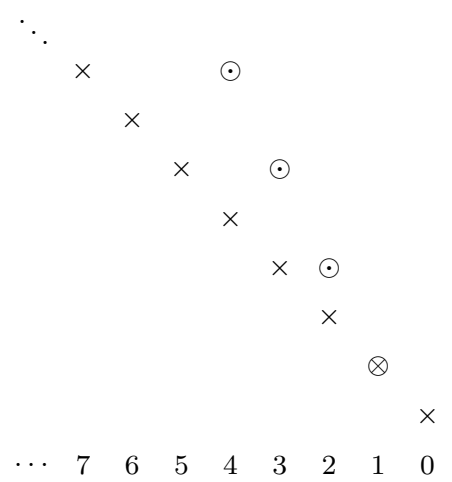

Fig. 14 Classes on the first page of the spectral sequence of $\dot{V}_{a}^{v}$. The numbers at the bottom are the number of hairs $h$, while the degree is $d=e+1=a-h+1$. On the vertical axis is the total valence of non-chosen vertices $s$, the number on which we set up the spectral sequence, starting at $2 a-1$ for $h=0$. Classes of the second type are labelled by $\times$ and classes of the first type are labelled by $\odot$. The position where there are both classes is labelled by $\otimes$.

On the second page of the spectral sequence we have a complex of second-type classes and first type classes with one hair, while the other first type classes are isolated. Let $\left(\mathrm{C}, \Delta_{1}\right)$ be the complex spanned by second-type classes of the second page of the spectral sequence. It is easily seen that $\dot{c}:\left(\mathrm{H}^{\geq 1} \bar{V}_{a}^{v-1}, \Delta\right) \rightarrow$ $\left(\mathrm{C}, \Delta_{1}\right)$ is an isomorphism of degree 1 , so we can replace $\left(\mathrm{C}, \Delta_{1}\right)$ with $\left(\mathrm{H}^{\geq 1} \bar{V}_{a}^{v-1}[-1], \Delta\right)$.

Proof of Proposition 7.3. Here we study the case $v=3$. No graphs satisfying $a<2$ exist. For $a=2$ whole $V_{2}^{3}$ is 1-dimensional, generated by $\rho_{2}=-\sigma_{1} \cup \lambda_{1}$. Hence, the cohomology is 1-dimensional, the class being represented by the same element, as expected.

If $a>2$ on the first page of the spectral sequence there is only one class of the first type, namely $\lambda_{1} \dot{\cup} \sigma_{a-1}$ of degree 2. It does not intrude the line with the second type classes and survives until the second page. On the second page in the line of second-type classes we have the complex isomorphic to $\mathrm{H}^{\geq 1} V_{a}^{2}[1]$, what is equal to $V_{a}^{2}[1]$ in this case. So, by Proposition 7.2 there is a class represented by $\dot{c}\left(\lambda_{a}\right)$ of degree 3 for odd $a$, and no class for even $a$.

Lemma $B .3$ shows that for odd $a$ the two classes cancel on further pages, so $\dot{V}_{3}^{a}$ is acyclic. Proposition A.2 implies that $V_{3}^{a}$ is acyclic too.

For even $a$ the class in the degree 2 survives. The element $\rho_{a}$ in $V_{3}^{a}$ (defined as a space of invariants) is also an element of $\dot{V}_{3}^{a}$ where it is equal to the sum over all vertices to be chosen in $\rho_{a}$. It contains $\lambda_{1} \dot{\cup} \sigma_{a-1}$ that forms a class that survives all pages, and since $\Delta\left(\rho_{a}\right)=0$ (Lemma B.4), $\rho_{a}$ represents the class in $\mathcal{H}\left(\dot{V}_{3}^{a}, \Delta\right)$. Proposition A.2 implies that it is also a class in $\mathcal{H}\left(V_{3}^{a}\right)$. That concludes the proposition.

Proof of Proposition 7.4. For $v \geq 4$ we prove by induction that $\mathcal{H}^{a-h+1}\left(V_{a}^{v}, \Delta\right)=0$ for $h \geq 1$. We prove the base $(v=4)$ and the step together.

We continue studying the spectral sequence on $\left(\dot{V}_{a}^{v}, \Delta\right)$ on the total valence $s$ of non-chosen vertices whose first page is depicted on Figure 14. Isolated first-type classes survive the second page, while the line of second-type classes and first-type classes with one hair is depicted on Figure 15. The second row represents the second type classes $\left(\mathrm{H}^{\geq 1} \bar{V}_{a}^{v-1}[-1], \Delta\right)$ as discussed above. But the $(a+1)$-degree term $\mathrm{H}^{1} V_{v-1}^{a}[1]$ is split into the sum $\operatorname{Ker}_{\Delta}\left(\mathrm{H}^{1} V_{v-1}^{a}\right) \oplus \operatorname{Im}_{\Delta}\left(\mathrm{H}^{1} V_{v-1}^{a}\right)$, and we only leave the first term in the second row, while we put the first term in the first row. The other term in the first row is in degree $a$ and represents the first type classes $\Gamma \dot{\mathrm{U}} \sigma_{1}$ for $\Gamma \in \mathrm{H}^{0} \bar{V}_{a-1}^{v-1}$.

There is a map that maps first-type class $\Gamma \dot{\cup} \sigma_{1}$ to the second-type class $\Delta_{1}\left(\Gamma \dot{\cup} \sigma_{1}\right)$ that is obtained from $\Gamma$ by adding an antenna in all possible ways, antenna ending with the chosen vertex. The isomorphic element in $\mathrm{H}^{1} \bar{V}_{a}^{v-1}[-1]$ is $\chi^{1}(\Gamma)$, where $\chi^{1}$ adds a hair in all possible ways, see $(2.19)$. That element is further split into $\chi_{1}^{1}(\Gamma) \in \operatorname{Ker}_{\Delta}\left(\mathrm{H}^{1} V_{a}^{v-1}\right)$ and $\chi_{0}^{1}(\Gamma)=\Delta\left(\chi^{1}(\Gamma)\right)=\nabla(\Gamma) \in \operatorname{Im}_{\Delta}\left(\mathrm{H}^{1} V_{a}^{v-1}\right) \subset \mathrm{H}^{0} V_{a}^{v-1}$ (see Lemma 2.5). 


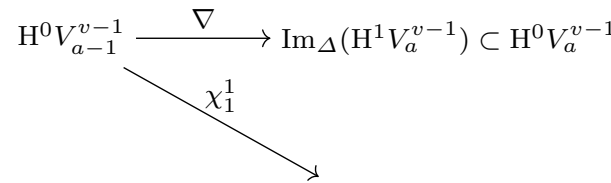

$$
\begin{aligned}
& \cdots \underset{\Delta}{\longrightarrow} \mathrm{H}^{5} V_{a}^{v-1}[-1] \underset{\Delta}{\longrightarrow} \mathrm{H}^{4} V_{a}^{v-1}[-1] \underset{\Delta}{\longrightarrow} \mathrm{H}^{3} V_{a}^{v-1}[-1] \underset{\Delta}{\longrightarrow} \mathrm{H}^{2} V_{a}^{v-1}[-1] \underset{\Delta}{\longrightarrow} \operatorname{Ker}_{\Delta}\left(\mathrm{H}^{1} V_{a}^{v-1}\right) \\
& \begin{array}{llllll}
a-3 & a-2 & a-1 & a & a+1
\end{array}
\end{aligned}
$$

Fig. 15 The complex on the second page of the spectral sequence.

Recall that we are not interested in the cohomology $\mathcal{H}^{a+1}\left(\dot{V}_{a}^{v}, \Delta\right)$. Classes in that degree of the complex on the first page from Figure 15 can not kill anything after the second page, so they can not change the existence of classes in other degrees. Therefore, we are not interested in classes of degree $a+1$ also in the complex from Figure 15

In that complex we set up the 2-row spectral sequence as in the figure, such that on the first page there is a complex with horizontal differentials, i.e. $\left(\mathrm{H}^{0} \bar{V}_{a-1}^{v-1} \stackrel{\nabla}{\rightarrow} \operatorname{Im}_{\Delta}\left(\mathrm{H}^{1} V_{a}^{v-1}\right)\right)$ and a reduced version of $\left(\bar{V}_{a}^{v-1}[-1], \Delta\right)$.

The cohomology of the first row in degree $a$ is clearly $\operatorname{Ker}_{\nabla}\left(\mathrm{H}^{0} V_{a-1}^{v-1}\right)$. The cohomology of the second row in relevant degrees $(d \leq a)$ is the same as $\mathcal{H}^{d}\left(V_{a}^{v-1}[-1], \Delta\right)$.

For the base of induction $(v=4)$ Proposition 7.3 implies that for even $a$ in the second row there is a class represented by $\rho_{a}$ in degree 3 . That class survives the spectral sequence because even if $a=2$ it cannot be killed by the first row, since in that case in the first row there is $\mathrm{H}^{0} V_{3}^{1}=0$.

For the step of the induction, assumption implies that the second row is acyclic. So, in any case cohomology has $\operatorname{Ker}_{\nabla}\left(\mathrm{H}^{0} V_{a-1}^{v-1}\right)$ in degree $a$, and the rest of the cohomology is zero except for $v=4$ and even $a$, when there is an extra class in degree 3 being represented by $\rho_{a}$.

On the third page of the spectral sequence we have a line of classes of the first type represented by $\Gamma \dot{\cup} \sigma_{h}$ for $\Gamma \in \mathrm{H}^{0} V_{a-h}^{v-1}$ in degrees $d \leq a-1$ and classes of the first type represented by $\Gamma \dot{\cup} \sigma_{1}$ for $\operatorname{Ker}_{\nabla}\left(\mathrm{H}^{0} V_{a-1}^{v-1}\right)$ in the degree $a$. All classes appear in even $s$ (the total valence of non-chosen vertices).

For $v=4$ and even $a$ the class $\dot{c}\left(\rho_{a}\right)$ has an odd $s$, so it must be outside of the mentioned line and it survives the third page.

Back to the line, if $h>1$ the whole differential $\Delta=\Delta_{0}+\Delta_{1}$ sends the representative $\Gamma \dot{\cup} \sigma_{h}$ of the class as pictured in Figure 16 .

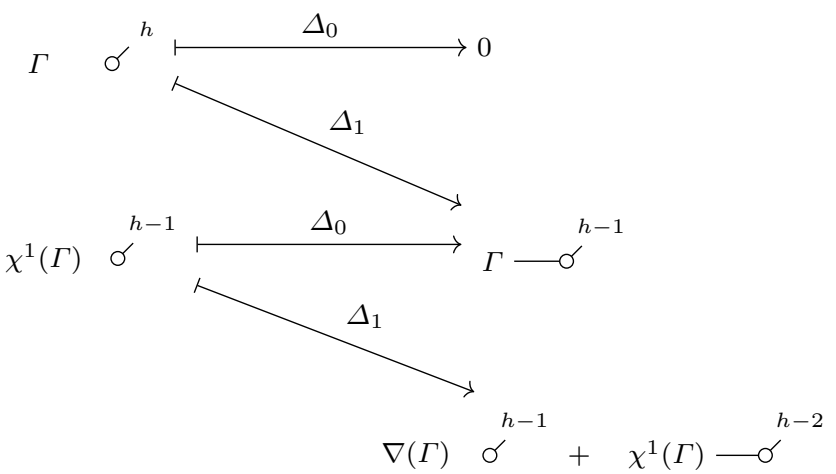

Fig. 16 Cancelling on the third page of the spectral sequence. The chosen vertex is marked with an empty circle.

The very last term does not represent a class on the first page (or does not exist when $h=1$ ), so on the third page of the spectral sequence there is the differential $\Gamma \cup \sigma_{h} \mapsto \nabla(\Gamma) \cup \sigma_{h-1}$. Even if $h=2$ the differential is well defined because it certainly ends in $\operatorname{Ker}_{\nabla}\left(\mathrm{H}^{0} V_{v-1}^{a-1}\right)$. So the complex on the third page looks like in Figure 17. 


$$
\cdots \stackrel{\nabla}{\longrightarrow} \mathrm{H}^{0} V_{v-1}^{a-5} \stackrel{\nabla}{\longrightarrow} \mathrm{H}^{0} V_{v-1}^{a-4} \stackrel{\nabla}{\longrightarrow} \mathrm{H}^{0} V_{v-1}^{a-3} \stackrel{\nabla}{\longrightarrow} \mathrm{H}^{0} V_{v-1}^{a-2} \stackrel{\nabla}{\longrightarrow} \operatorname{Ker}_{\nabla}\left(\mathrm{H}^{0} V_{v-1}^{a-1}\right)
$$

Fig. 17 Third page of the spectral sequence on $\dot{V}_{a}^{v}$.

This complex is a reduced version of $\left(\mathrm{V}^{v-1} \mathrm{fGC}_{0}^{\geq 1}, \nabla\right)$, and since $v-1 \neq 2$ the following lemma implies that it is acyclic.

Lemma 7.5. $\mathcal{H}\left(\mathrm{fGC}_{0}^{\geq 1}, \nabla\right)$ is 1-dimensional, the class being represented by $\lambda_{1}$.

Proof. Corollary [5, Corollary 3] clearly implies that $\mathcal{H}\left(\mathrm{fGCc}_{0}^{\geq 1}, \nabla\right)$ is one-dimensional, the class being represented by $\lambda_{1}$. On $\mathrm{fGC}_{0}^{\geq 1}$ we set up a spectral sequence on the number of connected components. The first differential does not change that number, so the cohomology is the symmetric product of $\lambda_{1}$. But there can not be more than one $\lambda_{1}$ because of the symmetry reasons, so the only class remaining is connected graph $\lambda_{1}$, concluding the proof.

If $v=4$ and $a>2$ even (for $a=2$ the class is out of interest because it is of degree $a+1$ ), there is one term left that survives on the third page, and therefore till the end: $\dot{c}\left(\rho_{a}\right)$. Analogously to Lemma 2.7 we have that $\dot{c} \Delta=\Delta \dot{c}$. Now, Lemma B.4 implies that $\Delta\left(\dot{c}\left(\rho_{a}\right)\right)=\dot{c}\left(\Delta\left(\rho_{a}\right)\right)=0$, so $\dot{c}\left(\rho_{a}\right)$ represents a class of the whole $\mathcal{H}\left(\dot{V}_{a}^{4}, \Delta\right)$. After taking invariants of the action of the symmetric group $S_{v}$ the class is sent to $c\left(\rho_{a}\right)$ by the symmetrization map, and it is zero because of Lemma B.5. Proposition A.2 implies that $\mathcal{H}\left(V_{a}^{4}, \Delta\right)=0$, concluding the base of the induction.

For the step, nothing survives the third page, so already $\dot{V}_{v}^{a}$ is acyclic, and because of Proposition A.2 $V_{v}^{a}$ is acyclic too. This finishes the step of the induction.

\subsection{The differential $\delta+\Delta$}

Propositions 7.1 to 7.4 imply that all classes of $\mathcal{H}\left(\mathrm{fHGC}_{-1,0}, \Delta\right)$ are in degrees 1 or 2 (have no or one edge), or hairless. We now want to change the differential to to $\delta+\Delta$ that exist because $\delta$ and $\Delta$ anti-commute (Lemma 2.4). With that differential there are no purely hairless classes, but we have the following proposition.

Proposition 7.6. Let $[\Gamma]$ be a non-trivial class of the cohomology $\mathcal{H}^{e+1}\left(\mathrm{fHGC}_{-1,0}, \delta+\Delta\right)$ for $e \geq 2$. Then the representative $\Gamma \in \mathrm{fHGC}_{-1,0}$ has a hairless part.

Proof. Suppose the opposite, that $\Gamma \in \mathrm{H}^{\geq 1} \mathrm{fHGC}_{-1,0}$. We write $\Gamma=\sum_{h \geq 1} \mathrm{H}^{h} \Gamma$ where $\mathrm{H}^{h} \Gamma$ is the part of $\Gamma$ with $h$ hairs. The equality $(\delta+\Delta)(\Gamma)=0$ separated by number of hairs imply that $\Delta\left(\mathrm{H}^{h} \Gamma\right)+$ $\delta\left(\mathrm{H}^{h-1} \Gamma\right)=0$ for every $h \geq 1$.

Particularly, for $h=1$ we get $\Delta\left(\mathrm{H}^{1} \Gamma\right)=0$. By Proposition $7.4\left(\mathrm{fHGC}_{-1,0}, \Delta\right)$ is acyclic at that degree, so there is $\mathrm{H}^{2} \gamma \in \mathrm{fHGC}_{-1,0}$ such that $\Delta\left(\mathrm{H}^{2} \gamma\right)=\mathrm{H}^{1} \Gamma . \Gamma_{2}:=\Gamma-(\delta+\Delta)\left(\mathrm{H}^{2} \gamma\right) \in \mathrm{H}^{\geq 2} \mathrm{fHGC}_{-1,0}$ is also a representative of $[\Gamma]$ in the starting complex.

For $h=2$ we get $\Delta\left(\mathrm{H}^{2} \Gamma\right)+\delta\left(\mathrm{H}^{1} \Gamma\right)=0$, implying that $\Delta\left(\mathrm{H}^{2} \Gamma_{2}\right)=0$. By the same argument there is $\mathrm{H}^{3} \gamma \in \mathrm{fHGC}_{-1,0}$ such that $\Delta\left(\mathrm{H}^{3} \gamma\right)=\mathrm{H}^{2} \Gamma_{2} . \Gamma_{3}:=\Gamma_{2}-(\delta+\Delta)\left(\mathrm{H}^{3} \gamma\right) \in \mathrm{H}^{\geq}{ }^{3} \mathrm{fHGC}_{-1,0}$ is another representative of $[\Gamma]$ in the starting complex, .

We can continue this argument to infinity and get $\mathrm{H}^{h} \gamma$ for $h \geq 2$ such that $\Gamma=(\delta+\Delta) \sum_{h \geq 2} \mathrm{H}^{h} \gamma$. This contradicts the assumption that $[\Gamma]$ is a non-trivial class.

Note that this proof is actually an elementary explanation of the spectral sequence argument where the first differential is $\Delta$. 


\subsection{Removing $\lambda$}

In this subsection we transform the result to the complex with the constraint $\dagger$ that does not have $\lambda=$ as a connected component. Recall that it does not have $\sigma_{0}=\bullet$ as a connected component either, because the minimal valence is 1 . Check that $\delta$ and $\Delta$ can not produce that connected components, so complexes with the the constraint $\dagger$ are closed under their action.

Proposition 7.7. Let $[\Gamma]$ be a non-trivial class of the cohomology $\mathcal{H}^{e+1}\left(\mathrm{fHGC}_{-1,0}^{\dagger}, \delta+\Delta\right)$ for $e \geq 1$. Then the representative $\Gamma \in \mathrm{fHGC}_{-1,0}^{\dagger}$ has a hairless part.

Note that the result is improved by including $e=1$, compared to Proposition 7.6

Proof. On $\left(\mathrm{fHGC}_{-1,0}, \delta+\Delta\right)$ we make the spectral sequence on the number of connected components $\lambda$. By symmetry reasons (exchanging hairs gives sign -1 ) there can be only one such connected component, so the spectral sequence has only two rows.

On the first page in the second row there is the complex of our interest $\left(\mathrm{fHGC}_{-1,0}^{\dagger}, \delta+\Delta\right)$. Graphs in the first row are of the form $\Gamma \cup \lambda$, where $\Gamma \in \mathrm{fHGC}_{-1,0}^{\dagger}$, together with $\lambda$ itself, and the first differential acts only within $\Gamma$. So the first row is isomorphic to $\left(\mathrm{fHGC}_{-1,0}^{\dagger}[-1] \oplus[\lambda], \delta+\Delta\right)$.

Let $\Gamma \cup \lambda$ represent a class on the first page in the first row. It is mapped to second row to $\Delta_{1}(\Gamma \cup \lambda)$ where $\Delta_{1}$ connects $\Delta$ and $\lambda$. It actually adds length-2 antenna instead of a hair to $\Gamma$, i.e. it is $2 c^{(2)}(\Gamma)$ (factor 2 comes from the two vertices in $\lambda$ to which $\Delta_{1}$ can connect) defined in 2.22 . Lemmas 2.6 and 2.7 imply that

$$
(\delta+\Delta) c(\Gamma)=c \delta(\Gamma)+c^{(2)}(\Gamma)+c \Delta(\Gamma)=c(\delta+\Delta)(\Gamma)+c^{(2)}(\Gamma)=c^{(2)}(\Gamma)
$$

because $(\delta+\Delta)(\Gamma)=0$. So, $\Delta_{1}(\Gamma \cup \lambda)=2 c^{(2)}(\Gamma)$ is exact in the second row and $\Gamma \cup \lambda-2 c(\Gamma)$ represents a class of $\mathcal{H}\left(\mathrm{fHGC}_{-1,0}, \delta+\Delta\right)$ coming from the first row. This shows that there are no cancellations between rows on the second page, and all classes from the first page survive the spectral sequence.

Now suppose that there is a non-trivial class $[\Gamma]$ of $\mathcal{H}^{e+1}\left(\mathrm{fHGC}_{-1,0}^{\dagger}, \delta+\Delta\right)$ for $e \geq 2$ without a hairless part. It sits in the second row of our spectral sequence, and can not be cancelled, so it represents also a class of $\mathcal{H}^{e+1}\left(\mathrm{fHGC}_{-1,0}, \delta+\Delta\right)$, contradicting Proposition 7.6. That was to be demonstrated for degrees $d \geq 3$.

Degree $e=1$ needs a special treatment. With 2 hairs there are no classes in both in the first row and in the second row. Recall that classes in the first row are generated by $\Gamma \cup \lambda$ where $\Gamma$ represents a class of $\mathcal{H}\left(\mathrm{fHGC}_{-1,0}^{\dagger}, \delta+\Delta\right)$ in degree 2 . By the construction above, the whole class of $\mathcal{H}^{3}\left(\mathrm{fHGC}_{-1,0}, \delta+\Delta\right)$ is represented by $\Gamma \cup \lambda-2 c(\Gamma)$. By Proposition 7.6 it has to have hairless part, so $\Gamma$ has to have a part with one hair.

There is only one graph in $\mathrm{fHGC}_{-1,0}^{\dagger}$ with 1 edge and 1 hair, namely $\lambda_{2}=\bullet \bullet \bullet$. So $\Gamma$ is of the form $\lambda_{2}+\Gamma^{\prime}$ where $\Gamma^{\prime}$ has all parts with more then 1 hair. But $\lambda_{2}$ is exact because $\lambda_{2}=(\delta+\Delta) \sigma_{1}$, so $\Gamma^{\prime}=\Gamma-(\delta+\Delta) \sigma_{1}$ represents the same class, contradicting the conclusion that it has to have a part with one hair.

\subsection{Reducing the complex}

In this subsection we reduce the complex to kill hairless classes. Let us define another complex

$$
\mathrm{H}^{\mathrm{b}} \mathrm{fHGC}_{-1,0}^{\dagger}:=\mathrm{H}^{\geq 1} \mathrm{fHGC}_{-1,0^{\dagger}} \oplus \mathrm{HL}_{0}
$$

where

$$
\mathrm{HL}_{0}:=\Delta\left(\mathrm{H}^{1} \mathrm{fHGC}_{-1,0}^{\dagger}\right)
$$

Since $\delta$ and $\Delta$ anti-commute (Lemma 2.4), $\mathrm{HL}_{0}$ is closed under the operation $\delta$. Therefore, the whole space $\mathrm{H}^{\mathrm{b}} \mathrm{fHGC}{ }_{-1,0}^{\dagger}$ is closed under $\delta+\Delta$ and $\left(\mathrm{H}^{\mathrm{b}} \mathrm{fHGC}_{-1,0}^{\dagger}, \delta+\Delta\right)$ is a subcomplex of $\left(\mathrm{fHGC}_{-1,0}^{\dagger}, \delta+\Delta\right)$. 
Proposition 7.8. All classes of the cohomology $\mathcal{H}\left(\mathrm{H}^{\mathrm{b}} \mathrm{fHGC}{ }_{-1,0}^{\dagger}, \delta+\Delta\right)$ consist of graphs with no edges, i.e. $\mathcal{H}^{e+1}\left(\mathrm{H}^{\mathrm{b}} \mathrm{fHGC}_{-1,0}^{\dagger}, \delta+\Delta\right)=0$ for $e \geq 1$.

Proof. Let $[\Gamma]$ be a non-trivial class in $\mathcal{H}^{e+1}\left(\mathrm{H}^{\mathrm{b}} \mathrm{fHGC}{ }_{-1,0}^{\dagger}, \delta+\Delta\right)$ for $e \geq 1$. If $\Gamma$ is exact in $\left(\mathrm{fHGC}_{-1,0}^{\dagger}, \delta+\Delta\right)$ then there is $\gamma \in \mathrm{fHGC}_{-1,0}^{\dagger}$ such that $(\delta+\Delta)(\gamma)=\Gamma$. But $\gamma$ must be also in $\mathrm{H}^{\mathrm{b}} \mathrm{fHGC}_{-1,0}^{\dagger}$, contradicting the assumption that $[\Gamma]$ be a non-trivial class. So $\Gamma$ represents a class also in $\left(\mathrm{fHGC}_{-1,0}^{\dagger}, \delta+\Delta\right)$. By Proposition $7.7 \Gamma$ has a hairless part $\mathrm{H}^{0} \Gamma \neq 0$.

Since $\Gamma \in \mathrm{H}^{b} \mathrm{fHGC}_{-1,0}^{\dagger}$ its hairless part must be in $\mathrm{HL}_{0}$, i.e. there exist $\gamma_{1} \in \mathrm{H}^{1} \mathrm{fHGC}_{-1,0}^{\dagger}$ such that $\Delta\left(\gamma_{1}\right)=\mathrm{H}^{0} \Gamma \cdot \Gamma-(\delta+\Delta)(\gamma)$ is also a representative of $[\Gamma]$, this time with zero hairless part, contradicting Proposition 7.7

\subsection{Bounded complex}

Recall from Definition 4.4 that bounded complex, the one decorated by $\ddagger$, is spanned by graphs that do not have $\lambda=\bullet \bullet, \sigma_{1}=\boldsymbol{\downarrow}$ or $\lambda_{2}=\bullet \bullet$ as a connected component. Let

$$
\mathrm{H}^{\mathrm{b}} \mathrm{fHGC}_{-1,0}^{\ddagger}:=\mathrm{H}^{\geq 1} \mathrm{fHGC}_{-1,0}^{\ddagger} \oplus \mathrm{HL}_{0} \subset \mathrm{H}^{\mathrm{b}} \mathrm{fHGC}_{-1,0}^{\dagger},
$$

where $\mathrm{HL}_{0}$ is as in (7.7).

Proposition 7.9. All classes of the cohomology $\mathcal{H}\left(\mathrm{H}^{\mathrm{b}} \mathrm{fHGC}_{-1,0}^{\ddagger}, \delta+\Delta\right)$ consist of the graphs that have no edges, i.e. $\mathcal{H}^{e+1}\left(\mathrm{H}^{\mathrm{b}} \mathrm{fHGC} \mathrm{C}_{-1,0}^{\ddagger}, \delta+\Delta\right)=0$ for $e \geq 1$.

Proof. On $\mathrm{H}^{\mathrm{b}} \mathrm{fHGC}_{-1,0}^{\dagger}$ we set up a spectral sequence of two rows: $\mathrm{fHGC}_{-1,0}^{\dagger / \ddagger}$ and $\mathrm{H}^{\mathrm{b}} \mathrm{fHGC}+1,0$. For the degree $d \geq 2(e \geq 1)$ the total complex is acyclic by Proposition 7.8 and in the first row by Proposition 4.13 there is only a class $[\alpha]$ in the degree 1 that go to zero by the whole differential, so it cannot cancel anything in the second row. That concludes the proof.

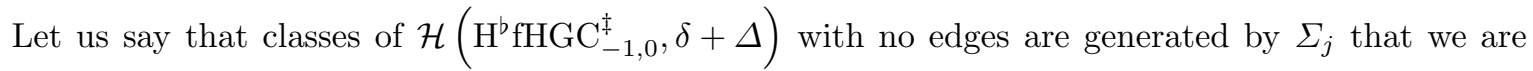
going to define in 7.9 . Since we do not need this fact later, we skip the proof.

7.6 The morphisms $\pi_{f}$

In this subsection we want to find a kind of map between two complexes, $\left(\mathrm{fGC}_{0}^{\geq 2}, \delta+D \nabla\right)$ and $\left(\mathrm{fHGC}_{-1,0}^{\ddagger}, \delta+\Delta\right)$ that will have a nice behaviour. Recall that $\delta+D \nabla$ is the conjugated differential $e^{D}(\delta+\nabla) e^{-D}$, see Proposition 3.10 A motivating example is depicted in Figure 18 .

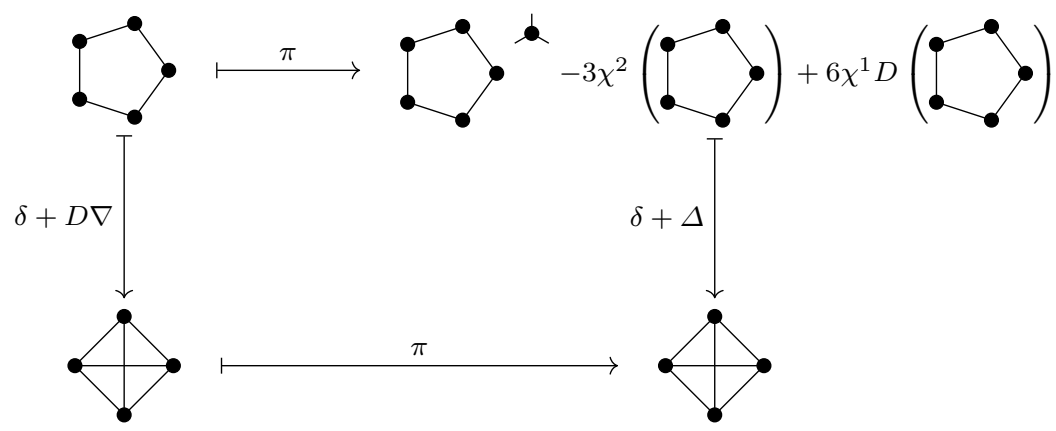

Fig. 18 A motivation for the map $\pi$ based on the equality from Figure 5 The right vertical mapping can be deduced from Lemma 2.5. Proposition 3.3. and equalities $\chi^{1} \delta=\delta \chi^{1}$ and $\chi^{2} \delta \Gamma-\delta \chi^{2} \Gamma=\frac{1}{3} \Delta\left(\Gamma \cup \sigma_{3}\right)$. Those equalities can be easily checked, or one can note that they are the simplest cases of Lemma B.7 
Recall that the differential $\delta+\Delta$ does not change the number $f=e-v+h$, so it is not surprising that $\pi$ from Figure 18 sends to the fixed subspace $\mathrm{F}^{f} \mathrm{fHGC}_{-1,0}^{\ddagger}$. The map $\pi$ possibly adds some stars, hairs or deletes vertices, in all cases increases $f$, so the target space $\mathrm{F}^{f} \mathrm{fHGC}_{-1,0}^{\ddagger}$ makes a restriction for the maximal $f$ of the starting graph $\Gamma \in \mathrm{fGC}_{0}^{\geq 2}$. In the non-hairy spaces $f$ is equal to $b=e-v$, so we deduce that $b$ has to be at most $f$ of the target space, and it will also be of the same parity. Therefore we will define maps

$$
\pi_{f}: \mathrm{B}^{\leq f, p a r} \mathrm{fGC}_{0}^{\geq 2} \rightarrow \mathrm{F}^{f} \mathrm{fHGC}_{-1,0}^{\ddagger},
$$

where prefix $\mathrm{B} \leq f$, par means all graphs with $b=e-v \leq f$ of the same parity as $f$.

Note that the subspace $\mathrm{B}^{\leq f \text {,par }} \mathrm{fGC}_{0}^{\geq 2} \subset \mathrm{fGC}_{0}^{\geq 2}$ is not closed under $\delta+D \nabla$, but its complement is. So, by complex $\left(\mathrm{B}^{\leq f, p a r} \mathrm{fGC}_{0}^{\geq 2}, \delta+D \nabla\right)$ we mean the complex with forbidden graphs identified with zero, or equivalently it is the quotient of $\left(\mathrm{fGC}_{0}^{\geq 2}, \delta+D \nabla\right)$ and the complement of $\mathrm{B} \leq f$, par $\mathrm{fGC}_{0}^{\geq 2}$.

We also see in example from Figure 18 that $\pi$ acts differently for different $b=e-v$. For the maximal possible $b(b=f) \pi$ is the identity. For $b=f-2 \pi$ adds a star $\sigma_{3}$ in the first term, and we can expect that there is a similar term for other $b$. For $b=f-4$, to reach the correct $f$, we can add two stars $\sigma_{3}$ or one star $\sigma_{5}$. We actually add

$$
\Sigma_{2}:=\frac{1}{2 \cdot 6^{2}} \not \boldsymbol{d}+\frac{-1}{5 !}
$$

The general definition of those elements for $j \geq 1$ is

$$
\Sigma_{j}:=\sum_{\substack{k_{i} \geq 0 \\ \sum_{i} i k_{i}=j}} \prod_{i>0} \frac{(-1)^{k_{i}}}{k_{i} !((2 i+1) !)^{k_{i}}} \bigcup_{i>0} \sigma_{2 i+1}^{\cup k_{i}} \in \mathrm{fHGC}_{-1,0}^{\ddagger} .
$$

Elements $\Sigma_{j}$ will mostly me used to make union with another graph. Although $\Sigma_{0}$ is not defined, we mean $\Gamma \cup \Sigma_{0}=\Gamma$.

Another example of $\Sigma_{j}$ is

$$
\Sigma_{3}=\frac{-1}{6 \cdot 6^{3}}
$$

The key property of $\Sigma_{j}$ is

$$
(\delta+\Delta)\left(\Sigma_{j}\right)=0
$$

as shown in Lemma B.6. The coefficients in 7.9 are indeed not surprising, they divide a graph with its order of symmetry (exchanging same stars and hairs in a star) such that coefficients from the operations disappear.

We are now ready to define maps $\pi_{f}$.

Definition 7.10. For every $f \in \mathbb{Z}$ we define degree-0 map $\pi_{f}: \mathrm{B}^{\leq f, p a r} \mathrm{fGC}_{0}^{\geq 2}[-1] \rightarrow \mathrm{F}^{f} \mathrm{H}^{\geq 1} \mathrm{fHGCd}_{-1,0}^{\ddagger}$. Let $\Gamma \in \mathrm{B}^{b} \mathrm{fGC}_{0}^{\geq 2}$ for $b=e-v \leq f$ of the same parity as $f$. Then

$$
\pi_{f}(\Gamma):=\sum_{i=0}^{\frac{f-b}{2}} \frac{1}{(2 i) !} \chi^{2 i}(\Gamma) \cup \Sigma_{\frac{f-b}{2}-i}-\sum_{i=1}^{\frac{f-b}{2}} \frac{1}{(2 i-1) !} \chi^{2 i-1} D(\Gamma) \cup \Sigma_{\frac{f-b}{2}-i} .
$$

Lemma B.8 implies that $\pi_{f}:\left(\mathrm{B}^{\leq f, p a r} \mathrm{fGC}_{0}^{\geq 2}[-1], \delta+D \nabla\right) \rightarrow\left(\mathrm{F}^{f} \mathrm{fHGC}_{-1,0}^{\ddagger}, \delta+\Delta\right)$ is a morphism of complexes for every $f \in \mathbb{Z}$.

\subsection{Connected complex}

In this subsection we transform the result to the connected complex. This result is much more complicated than the analogous result for odd case from Subsection 6.8. The problem this time is that $\left(\mathrm{H}^{\geq 1} \mathrm{fHGCc}_{-1,0}^{\ddagger}, \delta+\Delta\right)$ is not fully acyclic, there is a class represented by the star $\sigma_{3}$ as shows the following proposition. This makes the complex with disconnected graphs $\left(\mathrm{H}^{\geq 1} \mathrm{fHGC}_{-1,0}^{\ddagger}, \delta+\Delta\right)$ far from acyclic. Corollary 7.15 gives an insight to its cohomology, using map $\pi_{f}$ from the previous subsection. 
Therefore, the result that the complex with special hairless part $\left(\mathrm{H}^{b} \mathrm{fHGC}_{-1,0}^{\ddagger}, \delta+\Delta\right)$ is almost acyclic (Proposition 7.9) may be surprising. It seems that all disconnected classes are cancelled with the hairless part, leaving connected part acyclic. This is exactly what happens, as shown in the following proposition, particularly in its Claim IX,

Proposition 7.11. The cohomology $\mathcal{H}\left(\mathrm{H}^{\geq 1} \mathrm{fHGCc}_{-1,0}^{\ddagger}, \delta+\Delta\right)$ is one-dimensional, the class being represented by the star $\sigma_{3}$.

Proof. We prove the statement by the induction on the degree $d=e+1$.

The following lemma shows the claim in degrees $d=1$ and 2 .

\section{Lemma 7.12.}

$-\mathcal{H}^{1}\left(\mathrm{H}^{\geq 1} \mathrm{fHGCc}_{-1,0}^{\ddagger}, \delta+\Delta\right)$ is one dimensional, the class being generated by $\sigma_{3}=\boldsymbol{\alpha}$.

$-\mathcal{H}^{2}\left(\mathrm{H}^{\geq 1} \mathrm{fHGCc}_{-1,0}^{\ddagger}, \delta+\Delta\right)=0$.

Proof. Relevant graphs have at most 1 edge. Connected graphs with at most 1 edge are either a star, or a graph with 2 vertices and an edge between them. In both cases, second differential $\Delta$ does not do anything, and all graphs have $b=-1$. So we need to show the lemma for the complex $\left(\mathrm{B}^{-1} \mathrm{H}^{\geq 1} \mathrm{fHGCc}_{-1,0}^{\ddagger}, \delta\right)$. This shows Lemma 4.11 together with Corollary 4.10.

Let $d \geq 2$ and suppose that $\mathcal{H}\left(\mathrm{H}^{\geq 1} \mathrm{fHGCc}_{-1,0}^{\ddagger}, \delta+\Delta\right)$ is one-dimensional up to degree $d$, the class being represented by the star $\sigma_{3}$. We want to prove the same in degree $d+1$. We do it through a series of claims as follows.

Let $\Gamma \in \mathrm{fHGC}_{-1,0}^{\ddagger}$ be a graph. Let $p(\Gamma)$ be the number of tree-like connected components in $\Gamma$, i.e. connected components that have one vertex more than edges $(b=e-v$ of the connected component is -1 ). Differential $\delta$ acts within connected components and does not change $b$, so it can not change $p$. Differential $\Delta$ can save $p$ or decrease it by connecting a hair from tree-like connected component to something. Therefore, we can define a filtration on $\left(\mathrm{fHGC}_{-1,0}^{\ddagger}, \delta+\Delta\right)$ :

$$
\mathrm{P}^{\leq x} \mathrm{fHGC}_{-1,0}^{\ddagger} \subset \mathrm{fHGC}_{-1,0}^{\ddagger}
$$

spanned by all graphs $\Gamma$ such that $p(\Gamma) \leq x$. Let $\Delta_{P}$ be the part of $\Delta$ that saves $p$. It leaves tree-like connected components intact.

Claim I. $\mathcal{H}\left(\mathrm{H}^{\geq 1} \mathrm{fHGCc}_{-1,0}^{\ddagger}, \delta+\Delta_{P}\right)$ is one-dimensional up to degree $d$, the class being represented by the star $\sigma_{3}$.

Proof. We set up the spectral sequence of $\mathcal{H}\left(\mathrm{H}^{\geq 1} \mathrm{fHGCc}_{-1,0}^{\ddagger}, \delta+\Delta\right)$ on the number of tree-like connected components. A connected graph is either tree-like or it is not, so the spectral sequence has two rows on the first page:

$$
\begin{aligned}
& -\left(\mathrm{B}^{-1} \mathrm{H}^{\geq 1} \mathrm{fHGCc}_{-1,0}^{\ddagger}, \delta\right) \text { and } \\
& -\left(\mathrm{B}^{\geq 0} \mathrm{H}^{\geq 1} \mathrm{fHGCc}_{-1,0}^{\ddagger}, \delta+\Delta_{P}\right) .
\end{aligned}
$$

By Lemma 4.11 together with Corollary 4.10 the first row has 1-dimensional cohomology, the class being represented by the star $\sigma_{3}$. That class survives the spectral sequence, so classes of the second row have nothing to cancel with. Therefore, by the assumption, the second row is acyclic up to degree $d$. That implies the result.

Claim II. $\mathcal{H}\left(\mathrm{fHGCc}_{-1,0}^{\ddagger}, \delta+\Delta_{P}\right)$ is generated by $\left[\sigma_{3}\right]$ and classes of $\mathcal{H}\left(\mathrm{fGCc}_{0}^{\dagger}[-1], \delta\right)$, up to degree $d$.

Proof. We set up the spectral sequence of $\left(\mathrm{fHGCc}_{-1,0}^{\ddagger}, \delta+\Delta_{P}\right)$ of two rows on the first page:

$-\left(\mathrm{H}^{\geq 1} \mathrm{fHGCc}_{-1,0}^{\ddagger}, \delta+\Delta_{P}\right)$ (complex from Claim I and 
$-\left(\operatorname{fGCc}_{0}^{\dagger}[-1], \delta\right)$.

Class $\left[\sigma_{3}\right]$ from the first row survives the spectral sequence, implying the result.

Let $\Delta_{C P}$ be the part of $\Delta$ that fixes both number of tree-like connected components and the number of all connected components.

Claim III. $\mathcal{H}^{d}\left(\mathrm{fHGC}_{-1,0}^{\ddagger}, \delta+\Delta_{C P}\right)$ is generated by the classes represented by the degree-d union of a class representative of $\mathcal{H}\left(\mathrm{fGC}_{0}^{\dagger}[-1], \delta\right)$ and any number of $\sigma_{3}$.

Proof. The complex $\left(\mathrm{fHGC}_{-1,0}^{\ddagger}, \delta+\Delta_{C P}\right)$ is the symmetric product of the complex from Claim II $\left(\mathrm{fHGCc}_{-1,0}^{\ddagger}, \delta+\Delta_{P}\right)$ :

$$
\left(\mathrm{fHGC}_{-1,0}^{\ddagger}, \delta+\Delta_{C P}\right)=S^{+}\left(\mathrm{fHGCc}_{-1,0}^{\ddagger}, \delta+\Delta_{P}\right) .
$$

The cohomology commutes with the symmetric product and any of the factors in the symmetric product has the degree $\leq d$. Therefore $\mathcal{H}^{d}\left(\mathrm{fHGC}_{-1,0}^{\ddagger}, \delta+\Delta_{C P}\right)$ is generated by the classes represented by the degree- $d$ union of class representatives of $\mathcal{H}\left(\mathrm{fGCc}_{0}^{\dagger}[-1], \delta\right)$ and any number of $\sigma_{3}$. Since $d \geq 2(e \geq 1)$ there has to be at least one connected component that is not a star, so it is hairless.

Since $\mathcal{H}\left(\mathrm{fGC}_{0}^{\dagger}[-1], \delta\right)$ is the symmetric product of $\mathcal{H}\left(\mathrm{fGCc}_{0}^{\dagger}[-1], \delta\right)$, the union of hairless connected components forms a representative of a class of $\mathcal{H}\left(\mathrm{fGC}_{0}^{\dagger}[-1], \delta\right)$, implying the result.

Let us define a map $\pi_{f}^{0}: \mathrm{B} \leq f, p a r f \mathrm{fGC}_{0}^{\geq 2}[-1] \rightarrow \mathrm{F}^{f}$ fHGC ${ }_{-1,0}^{\ddagger}$ as

$$
\pi_{f}^{0}(\Gamma):=\Gamma \cup \sigma_{3}^{\cup \frac{f-b}{2}} .
$$

It is clearly $\Delta_{P} \pi_{f}^{0}(\Gamma)=\pi_{f}^{0} \delta(\Gamma)$, so the map $\pi_{f}^{0}$ is the map of complexes $\left(\mathrm{B}^{\leq f, p a r} \mathrm{fGC}_{0}^{\geq 2}[-1], \delta\right) \rightarrow$ $\left(\mathrm{F}^{f} \mathrm{fHGC}{ }_{-1,0}^{\ddagger}, \delta+\Delta_{P}\right)$. The same is true with $\Delta_{C P}$ instead of $\Delta_{P}$.

Claim IV. For every $f \geq 0$ the map $\pi_{f}^{0}:\left(\mathrm{B}^{\leq f, p a r} \mathrm{fGC}_{0}^{\geq 2}[-1], \delta\right) \rightarrow\left(\mathrm{F}^{f} \mathrm{fHGC}_{-1,0}^{\ddagger}, \delta+\Delta_{C P}\right)$ is a quasi-isomorphism in degree $d$.

Proof. The map $\pi_{f}^{0}$ is constructed exactly to map classes of $\left(\mathrm{B}^{\leq f, p a r} \mathrm{fGC}_{0}^{\dagger}[-1], \delta\right)$ to classes of $\left(\mathrm{F}^{f} \mathrm{fHGC} \mathrm{C}_{-1,0}^{\ddagger}, \delta+\Delta_{C P}\right)$ described in Claim III

Proposition 4.8 for hairless part and for fixed $b$ implies that the inclusion $\left(\mathrm{B}^{b} \mathrm{fGC}_{0}^{\geq 2}[-1], \delta\right) \hookrightarrow$ $\left(\mathrm{B}^{b} \mathrm{fGC}_{0}^{\dagger}[-1], \delta\right)$ is a quasi-isomorphism, implying the same for $\left(\mathrm{B}^{\leq f, p a r} \mathrm{fGC}_{0}^{\geq 2}[-1], \delta\right) \hookrightarrow$ $\left(\mathrm{B}^{\leq f, p a r} \mathrm{fGC}_{0}^{\dagger}[-1], \delta\right)$. This implies the result.

Claim V. For every $f \geq 0$ the map $\pi_{f}^{0}:\left(\mathrm{B}^{<f, p a r} \mathrm{fGC}_{0}^{\geq 2}[-1], \delta\right) \rightarrow\left(\mathrm{F}^{f} \mathrm{H}^{\geq 1} \mathrm{fHGC}_{-1,0}^{\ddagger}, \delta+\Delta_{C P}\right)$ is a quasi-isomorphism in degree $d$.

Note that we have restricted $\pi_{f}^{0}$ to $\mathrm{B}^{<f, p a r} \mathrm{fGC}_{0}^{\geq 2}[-1]$, where prefix $\mathrm{B}^{<f, p a r}$ means all graphs with $b=e-v<f$ of the same parity as $f$. The image of this restriction always has at least one hairy component $\sigma_{3}$, so we can co-restrict it to $\mathrm{F}^{f} \mathrm{H}^{\geq 1} \mathrm{fHGC}_{-1,0}^{\ddagger}$.

Proof. On the mapping cone of the map $\pi_{f}^{0}:\left(\mathrm{B}^{\leq f, p a r} \mathrm{fGC}_{0}^{\geq 2}[-1], \delta\right) \rightarrow\left(\mathrm{F}^{f} \mathrm{fHGC}_{-1,0}^{\ddagger}, \delta+\Delta_{C P}\right)$ from Claim IV we set up the spectral sequence of two rows on the first page:

- the mapping cone of $\pi_{f}^{0}:\left(\mathrm{B}^{<f, p a r} \mathrm{fGC}_{0}^{\geq 2}[-1], \delta\right) \rightarrow\left(\mathrm{F}^{f} \mathrm{H}^{\geq 1} \mathrm{fHGC}_{-1,0}^{\ddagger}, \delta+\Delta_{C P}\right)$ and 
- the mapping cone of the inclusion $\left(\mathrm{B}^{f} \mathrm{fGC}_{0}^{\geq 2}[-1], \delta\right) \hookrightarrow\left(\mathrm{B}^{f} \mathrm{fGC}_{0}^{\dagger}[-1], \delta\right)$.

By Proposition 4.8 the latter map is a quasi-isomorphism, so the second row is acyclic. Therefore Claim IV] implies the result.

Let

$$
\text { fHGCd }_{-1,0} \subset \text { fHGC }_{-1,0}
$$

be the subset spanned by disconnected graphs. The image of the restriction $\pi_{f}^{0}: \mathrm{B}^{<f, p a r} \mathrm{fGC}_{0}^{\geq 2}[-1] \rightarrow$ $\mathrm{F}^{f} \mathrm{H}^{\geq 1} \mathrm{fHGC}_{-1,0}^{\ddagger}$, having at least one hairy component $\sigma_{3}$, can be further co-restricted to the disconnected part $\mathrm{F}^{f} \mathrm{H}^{\geq 1} \mathrm{fHGCd}_{-1,0}^{\ddagger}$. We have the following claim.

Claim VI. For every $f \geq 0$ the map $\pi_{f}^{0}:\left(\mathrm{B}^{<f, p a r} \mathrm{fGC}_{0}^{\geq 2}[-1], \delta\right) \rightarrow\left(\mathrm{F}^{f} \mathrm{H}^{\geq 1} \mathrm{fHGCd}_{-1,0}^{\ddagger}, \delta+\Delta_{C P}\right)$ is a quasi-isomorphism in degree $d$.

Proof. By definition $\Delta_{C P}$ can not reduce the number of connected components, so we have a direct sum of complexes

$$
\left(\mathrm{F}^{f} \mathrm{H}^{\geq 1} \mathrm{fHGC}_{-1,0}^{\ddagger}, \delta+\Delta_{C P}\right)=\left(\mathrm{F}^{f} \mathrm{H}^{\geq 1} \mathrm{fHGCc}_{-1,0}^{\ddagger}, \delta+\Delta_{C P}\right) \oplus\left(\mathrm{F}^{f} \mathrm{H}^{\geq 1} \mathrm{fHGCd}_{-1,0}^{\ddagger}, \delta+\Delta_{C P}\right) .
$$

The map $\pi_{f}^{0}$ maps exclusively to the second addend, so Claim V implies the result.

Claim VII. For every $f \geq 0$ the map $\pi_{f}^{0}:\left(\mathrm{B}^{<f, p a r} \mathrm{fGC}_{0}^{\geq 2}[-1], \delta\right) \rightarrow\left(\mathrm{F}^{f} \mathrm{H}^{\geq 1} \mathrm{fHGCd}_{-1,0}^{\ddagger}, \delta+\Delta_{P}\right)$ is a quasi-isomorphism in degree $d$.

Proof. On the mapping cone of $\pi_{f}^{0}:\left(\mathrm{B}^{<f, p a r} \mathrm{fGC}_{0}^{\geq 2}[-1], \delta\right) \rightarrow\left(\mathrm{F}^{f} \mathrm{H}^{\geq 1} \mathrm{fHGCd}_{-1,0}^{\ddagger}, \delta+\Delta_{P}\right)$ we set up a spectral sequence on the number of connected components, such that on the first page is the mapping cone of the map from Claim VI. For fixed $b$ and $f$ this spectral sequence is bounded, hence converges correctly. Claim VI implies the result.

We split the map $\pi_{f}$ into connected and disconnected part: $\pi_{f}(\Gamma)=\pi_{f}^{c o n n}(\Gamma)+\pi_{f}^{\text {disconn }}(\Gamma)$ where $\pi_{f}^{\text {conn }}: \mathrm{B}^{<f, p a r} \mathrm{fGC}_{0}^{\geq 2}[-1] \rightarrow \mathrm{F}^{f} \mathrm{H}^{\geq 1} \mathrm{fHGCc}_{-1,0}^{\ddagger}$ and $\pi_{f}^{\text {disconn }}: \mathrm{B}^{<f, p a r} \mathrm{fGC}_{0}^{\geq 2}[-1] \rightarrow \mathrm{F}^{f} \mathrm{H}^{\geq 1} \mathrm{fHGCd}_{-1,0}^{\ddagger}$. Explicitly, using (7.11, for connected graph $\Gamma \in \mathrm{B}^{<f, p a r} \mathrm{fGCc}_{0}^{\geq 2}[-1]$

$$
\begin{gathered}
\pi_{f}^{d i s c o n n}(\Gamma):=\sum_{i=0}^{\frac{f-b}{2}-1} \frac{1}{(2 i) !} \chi^{2 i}(\Gamma) \cup \Sigma_{\frac{f-b}{2}-i}-\sum_{i=1}^{\frac{f-b}{2}-1} \frac{1}{(2 i-1) !} \chi^{2 i-1} D(\Gamma) \cup \Sigma_{\frac{f-b}{2}-i}, \\
\pi_{f}^{c o n n}(\Gamma)=\frac{1}{(f-b) !} \chi^{f-b}(\Gamma)-\frac{1}{(f-b-1) !} \chi^{f-b-1} D(\Gamma),
\end{gathered}
$$

and for disconnected graph $\Gamma \in \mathrm{B}^{<f, p a r} \mathrm{fGC}_{0}^{\geq 2}[-1]$ it is $\pi_{f}^{\text {disconn }}(\Gamma)=\pi_{f}$ and $\pi_{f}^{\text {conn }}(\Gamma)=0$.

Claim VIII. For every $f \geq 0$ the map $\pi_{f}^{\text {disconn }}:\left(\mathrm{B}^{<f, p a r} \mathrm{fGC}_{0}^{\geq 2}[-1], \delta+D \nabla\right) \rightarrow$ $\left(\mathrm{F}^{f} \mathrm{H}^{\geq 1} \mathrm{fHGCd}_{-1,0}^{\ddagger}, \delta+\Delta\right)$ is a quasi-isomorphism in degree $d$.

Proof. On $\left(\mathrm{B}^{<f, p a r} \mathrm{fGC}_{0}^{\geq 2}[-1], \delta+D \nabla\right)$ we consider the filtration on $x \mathrm{~B}^{\geq f-2 x} \mathrm{~B}^{<f, p a r} \mathrm{fGC}_{0}^{\geq 2}[-1]$, and on $\left(\mathrm{F}^{f} \mathrm{H}^{\geq 1} \mathrm{fHGCd}_{-1,0}^{\ddagger}, \delta+\Delta\right)$ we consider the filtration on the number of tree-like connected components $\mathrm{P} \leq x \mathrm{~F}^{f} \mathrm{H}^{\geq 1} \mathrm{fHGCd}_{-1,0}^{\ddagger}$. We have the following lemma.

Lemma 7.13. For every $f \geq 0$ the map $\pi_{f}^{\text {disconn }}$ respects the filtrations $\mathrm{B}^{\geq f-2 x} \mathrm{~B}^{<f, p a r} \mathrm{fGC}_{0}^{\geq 2}[-1]$ and $\mathrm{P}^{\leq x} \mathrm{~F}^{f} \mathrm{H}^{\geq 1} \mathrm{fHGCd}_{-1,0}^{\ddagger}$, i.e. for every $x \in \mathbb{Z} \pi_{f}\left(\mathrm{~B}^{\geq f-2 x} \mathrm{~B}^{<f, p a r} \mathrm{fGC}_{0}^{\geq 2}[-1]\right) \subset \mathrm{P}^{\leq x} \mathrm{~F}^{f} \mathrm{H}^{\geq 1} \mathrm{fHGCd}_{-1,0}^{\ddagger}$. 
Proof. Let $\Gamma \in \mathrm{B}^{\geq f-2 x} \mathrm{~B}^{<f, p a r} \mathrm{fGC}_{0}^{\geq 2}[-1]$ be a graph with $v$ vertices and $e$ edges. All those constraints mean that $b=e-v$ is of the same parity as $f$ and $f-2 x \leq b<f$.

Tree-like hairless connected component needs to have 1 -valent vertex, so there are none in $\Gamma$. Adding hairs can not change this. All connected components of addends of $\Sigma_{j}(7.9)$ are tree like, and there are at most $j$ of them.

Therefore, the first sum in $\pi_{f}^{\text {disconn }}(\Gamma)$, 7.15) or 7.11, has the most number of tree-like connected components for $i=0$ and it is $\frac{f-b}{2} \leq x$. The second sum has even less hairy connected components, hence the result.

On the mapping cone of $\pi_{f}^{\text {disconn }}:\left(\mathrm{B}^{\leq f, p a r} \mathrm{fGC}_{0}^{\geq 2}[-1], \delta+D \nabla\right) \rightarrow\left(\mathrm{F}^{f} \mathrm{H}^{\geq 1} \mathrm{fHGCd}_{-1,0}^{\ddagger}, \delta+\Delta\right)$ we construct the filtration from the lemma. The first differentials of the complexes are $\delta$, respectively $\delta+\Delta_{P}$. On the first page we have the mapping cone of the map $\left(\mathrm{B}^{<f, p a r} \mathrm{fGC}_{0}^{\geq 2}[-1], \delta\right) \rightarrow$ $\left(\mathrm{F}^{f} \mathrm{H}^{\geq 1} \mathrm{fHGCd}_{-1,0}^{\ddagger}, \delta+\Delta_{P}\right)$ that sends $\Gamma$ with $b=f-2 x$ to the part of $\pi_{f}(\Gamma)$ with $x$ tree-like connected components. Only addend in $\pi_{f}^{\text {disconn }}(\Gamma)$, 7.15) or (7.11), with that much tree-like connected components is $C \Gamma \cup \sigma_{3}^{\cup \frac{f-b}{2}}=C \pi_{f}^{0}(\Gamma)$ where $C$ is an irrelevant coefficient. So, it is the mapping cone of the map from Claim VII. The spectral sequence is bounded, so it converges correctly and Claim VII implies the result.

Claim IX. The claim of the induction holds in degree $d+1$, i.e. $\mathcal{H}^{d+1}\left(\mathrm{H}^{\geq 1} \mathrm{fHGCc}_{-1,0}^{\ddagger}, \delta+\Delta\right)=0$.

Proof. On the complex from Proposition $7.9\left(\mathrm{H}^{b} \mathrm{fHGC}_{-1,0}^{\ddagger}, \delta+\Delta\right)$ we set up a spectral sequence of three rows:

$-\left(\mathrm{H}^{\geq 1} \mathrm{fHGCd}_{-1,0}^{\ddagger}, \delta+\Delta\right)$ (complex from Claim VIII),

- $\left(\mathrm{H}^{\geq 1} \mathrm{fHGCc}_{-1,0}^{\ddagger}, \delta+\Delta\right)$ (complex we are interested in) and

$-\left(\mathrm{HL}_{0}, \delta\right)$.

Proposition 7.9 implies that the whole complex is acyclic in degree $d+1$, so all classes of the first page in that degree cancel on further pages.

Let us take a class in the first row of degree $d$. Claim VIII implies that it is generated by $\pi_{f}^{\text {disconn }}(\Gamma)$ for some $f \in \mathbb{Z}$ and $\Gamma$ being a class representative of a class in $\mathcal{H}\left(\mathrm{B}^{<f, p a r} \mathrm{fGC}_{0}^{\geq 2}[-1], \delta+D \nabla\right)$. In particular $(\delta+D \nabla)(\Gamma)=0$.

That representative is sent to $(\delta+\Delta) \pi_{f}^{\text {disconn }}(\Gamma)$ in the second row. Lemma B.8 implies that $(\delta+$ $\Delta) \pi_{f}(\Gamma)=(\delta+\Delta) \pi_{f}^{d i s c o n n}(\Gamma)+(\delta+\Delta) \pi_{f}^{c o n n}(\Gamma)=0$, so $(\delta+\Delta) \pi_{f}^{d i s c o n n}(\Gamma)$ is exact in the second row, and can not represent a class. The class was chosen arbitrary, so we conclude that there are no cancellations between first row in degree $d$ and the second row in degree $d+1$. The following lemma says that there are also no cancellations between second and third row.

Lemma 7.14. In the spectral sequence of $\left(\mathrm{H}^{b} \mathrm{fHGC}_{-1,0}^{\ddagger}, \delta+\Delta\right)$ containing rows $\mathrm{H}^{\geq} \mathrm{fHGCd}_{-1,0}^{\ddagger}$, $\mathrm{H}^{\geq 1} \mathrm{fHGCc}_{-1,0}^{\ddagger}$ and $\mathrm{HL}_{0}$ classes of $\mathcal{H}\left(\mathrm{H}^{\geq 1} \mathrm{fHGCc}_{-1,0}^{\ddagger}, \delta+\Delta\right)$ and $\mathcal{H}\left(\mathrm{HL}_{0}, \delta\right)$ from the first page do not cancel on the second page.

Proof. Suppose the opposite, i.e. there is $\Gamma \in \mathrm{H}^{\geq 1} \mathrm{fHGCc}_{-1,0}^{\ddagger}$ and $\gamma \in \mathrm{HL}_{0}$ that represent classes in $\mathcal{H}\left(\mathrm{H}^{\geq 1} \mathrm{fHGCc}_{-1,0}^{\ddagger}, \delta+\Delta\right)$, respectively $\mathcal{H}\left(\mathrm{HL}_{0}, \delta\right)$, and that cancel each other. Then, $(\delta+\Delta) \Gamma$ is in the class of $\gamma$. We may choose $\gamma$ such that $(\delta+\Delta) \Gamma=\gamma$.

Let $\Gamma=\sum_{i \geq 1} \mathrm{H}^{i} \Gamma$ where $\mathrm{H}^{i} \Gamma$ is the part with $i$ hairs. Propositions 5.3 5.5 and 5.6 imply

$$
\begin{gathered}
\delta D^{(1)}\left(\mathrm{H}^{1} \Gamma\right)-D^{(1)} \delta\left(\mathrm{H}^{1} \Gamma\right)=\Delta\left(\mathrm{H}^{1} \Gamma\right), \\
2 \nabla\left(\delta D^{(2)}\left(\mathrm{H}^{2} \Gamma\right)+D^{(2)} \delta\left(\mathrm{H}^{2} \Gamma\right)\right)=D^{(1)} \Delta\left(\mathrm{H}^{2} \Gamma\right), \\
2 \nabla D^{(2)} \Delta\left(\mathrm{H}^{3} \Gamma\right)=0 .
\end{gathered}
$$


Summing all those equalities using $\delta \nabla=-\nabla \delta$ implies

$$
\begin{aligned}
\delta\left(D^{(1)}\left(\mathrm{H}^{1} \Gamma\right)-2 \nabla D^{(2)}\right. & \left.\left(\mathrm{H}^{2} \Gamma\right)\right)= \\
& =D^{(1)}\left(\delta\left(\mathrm{H}^{1} \Gamma\right)+\Delta\left(\mathrm{H}^{2} \Gamma\right)\right)-2 \nabla D^{(2)}\left(\delta\left(\mathrm{H}^{2} \Gamma\right)+\Delta\left(\mathrm{H}^{3} \Gamma\right)\right)+\Delta\left(\mathrm{H}^{1} \Gamma\right) .
\end{aligned}
$$

Equality $(\delta+\Delta) \Gamma=\gamma$ separated by the number of hairs says $\Delta\left(\mathrm{H}^{1} \Gamma\right)=\gamma$ and $\delta\left(\mathrm{H}^{i} \Gamma\right)+\Delta\left(\mathrm{H}^{i+1} \Gamma\right)=0$ for $i \geq 2$, so

$$
\delta\left(D^{(1)}\left(\mathrm{H}^{1} \Gamma\right)-2 \nabla D^{(2)}\left(\mathrm{H}^{2} \Gamma\right)\right)=\gamma .
$$

Lemma 5.1 and definition (5.4) imply that

$$
D^{(1)}\left(\mathrm{H}^{1} \Gamma\right)+D^{(2)}\left(\mathrm{H}^{2} \Gamma\right)=D^{(1)}\left(\mathrm{H}^{1} \Gamma\right)+D^{(1)} D^{(2-1)}\left(\mathrm{H}^{2} \Gamma\right)=\nabla D^{(p u s h)}\left(\left(\mathrm{H}^{1} \Gamma\right)+D^{(2-1)}\left(\mathrm{H}^{2} \Gamma\right)\right)
$$

so it is in $\mathrm{HL}_{0}$, and $\gamma$ is exact in $\left(\mathrm{HL}_{0}, \delta\right)$, contradicting the assumption.

Therefore, a class in the middle row at degree $d+1$, i.e. in $\mathcal{H}^{d+1}\left(\mathrm{H}^{\geq 1} \mathrm{fHGCc}_{-1,0}^{\ddagger}, \delta+\Delta\right)$, can not cancel with anything. Since everything cancels, there can not be a class in $\mathcal{H}^{d}\left(\mathrm{H}^{\geq 1} \mathrm{fHGCc}_{-1,0}^{\ddagger}, \delta+\Delta\right)$. That was to be demonstrated in this claim.

The last claim finishes the step of the induction. That was to be demonstrated in this proposition.

Corollary 7.15. The map $\pi_{f}:\left(\mathrm{B}^{\leq f, p a r} \mathrm{fGC}_{0}^{\geq 2}[-1], \delta+D \nabla\right) \rightarrow\left(\mathrm{F}^{f} \mathrm{fHGC}_{-1,0}^{\ddagger}, \delta+\Delta\right)$ is a quasiisomorphism in every degree $d \geq 2(e \geq 1)$ for every $f \in \mathbb{Z}$.

Proof. Since the induction in the proof of Proposition 7.11 is shown, all its claims hold for every $d \geq 2$. We use Claim $\operatorname{IV}\left(\pi_{f}^{0}\right.$ is quasi-isomorphism with differential $\left.\delta+\Delta_{C P}\right)$, skip Claims $\mathrm{V}$ (removing hairless part) and VI (removing connected part), and the analogous arguments as in Claims VII (transforming $\Delta_{C P}$ to $\Delta_{P}$ ) and VIII (transforming $\pi_{f}^{0}$ to $\pi_{f}^{d i s c o n n}$, this time to $\pi_{f}$ ) imply the result.

We finish the proof of Theorem 1.1 with the following proposition. It extends the result of Corollary 4.10 to the extra differential. The proof uses standard spectral sequence argument that sees a known differential at the first page.

Proposition 7.16. The inclusions

$$
\left(\mathrm{HGC}_{-1, n}, \delta+\Delta\right) \hookrightarrow\left(\mathrm{H}^{\geq 1} \mathrm{fHGCc}_{-1, n}^{\geq 2}, \delta+\Delta\right) \hookrightarrow\left(\mathrm{H}^{\geq 1} \mathrm{fHGCc}_{-1, n}^{\ddagger}, \delta+\Delta\right)
$$

are quasi-isomorphisms.

Proof. First of all we split all complexes as in 2.30):

$$
(\mathrm{C}, \delta+\Delta)=\prod_{f \in \mathbb{Z}}\left(\mathrm{F}^{f} \mathrm{C}, \delta+\Delta\right)
$$

where $f=e+h-v$. On the mapping cone of any inclusion we set up a spectral sequence on the number $e-v$. One checks that in each degree $d=1+v n+(1-n) e-n h$ the spectral sequence is bounded, so it converges correctly. On the first page of the spectral sequence there is a mapping cone of the inclusion of complexes only with the standard differential $\delta$, so it is acyclic by Corollary 4.10 . Therefore the whole mapping cone is acyclic, and the inclusion is quasi-isomorphism.

Now Propositions 7.11 and 7.16 conclude the proof of the second part of Theorem 1.1 saying that the cohomology $\mathcal{H}\left(\mathrm{HGC}_{-1,0}, \delta+\Delta\right)$ is one-dimensional, the class being represented by $\sigma_{3}$. 


\section{A Group action}

In this section of appendix we clarify one way of calculating cohomology of a graph complex, by doing so first with distinguishing some or all vertices.

In defining graph complexes we start with a graph with distinguishable elements (vertices, edges, hairs). The graph complex is the space of invariants of a finite group that permutes elements acting on the starting space (recall subsections 2.2 and 2.4. Since the groups are finite, the space of invariants is isomorphic to the space of coinvariants. For the simplicity we work with the former space.

In this paper we are interested only in the action of the symmetric group $S_{v}$ that permutes vertices, taking as a starting space the space that already is the space of invariants of the action of the other groups. The same can similarly be done with the other elements of the graph.

The space of invariants of the action $\rho$ of finite group $G$ on the space $V$ is

$$
V^{G}=\left\{\gamma \in V \mid \rho_{g}(\gamma)=\gamma \text { for all } g \in G\right\}
$$

Let $(V, d)$ be a complex and $G$ a finite group acting on $\mathrm{C}$ by the action of degree $0 \rho_{g}: \mathrm{C} \rightarrow \mathrm{C}$ for $g \in G$. Let the action and the differential commute, i.e.

$$
\rho_{g} d(\gamma)=d \rho_{g}(\gamma)
$$

for every $g \in G$ and $\gamma \in \mathrm{C}$. The action of the group can be extended to cohomology of C as $\rho_{g}([\gamma])=\left[\rho_{g}(\gamma)\right]$ for $[\gamma] \in \mathcal{H}(\mathrm{C})$.

Proposition A.1. Let a finite group $G$ act on a vector space $C$ over a field of characteristic zero. Then

$$
\mathcal{H}\left(\mathrm{C}^{G}, d\right)=\mathcal{H}(\mathrm{C}, d)^{G} .
$$

Proof. Standard result.

In particular, we are interested in the graph complex $(\mathrm{C}, d)$ where $d$ does not change the number of vertices, i.e.

$$
(\mathrm{C}, d)=\prod_{v}\left(\mathrm{~V}^{v} \mathrm{C}, d\right) .
$$

Let $\overline{\mathrm{V}}^{v} \mathrm{C}$ be the space with distinguishable vertices, before taking invariants of $S_{v}$. E.g. for hairy graph complexes it is

$$
\overline{\mathrm{V}}^{v} \mathrm{H}^{h} \mathrm{fHGC}_{-1, n}^{\geq i}:= \begin{cases}\prod_{e}\left(V_{v, e, h}^{\geq i} \otimes \operatorname{sgn}_{e}\right)^{S_{h} \times\left(S_{e} \ltimes S_{2}^{\times e}\right)} & \text { for } n \text { even, } \\ \prod_{e}\left(V_{v, e, h}^{\geq i} \otimes \operatorname{sgn}_{v} \otimes \operatorname{sgn}_{h} \otimes \operatorname{sgn}_{2}^{\otimes e}\right)^{S_{h} \times\left(S_{e} \ltimes S_{2}^{\times e}\right)} & \text { for } n \text { odd },\end{cases}
$$

c.f. definition 2.9 It is always

$$
\mathrm{V}^{v} \mathrm{C}=\left(\overline{\mathrm{V}}^{v} \mathrm{C}\right)^{S_{v}}
$$

and the differential on $\mathrm{V}^{v} \mathrm{C}$ that does not change the number of vertices is induced from the one on $\overline{\mathrm{V}}^{v} \mathrm{C}$. The proposition A.1 gives us an easy tool to calculate the cohomology of the graph complex:

$$
\mathcal{H}(\mathrm{C}, d)=\prod_{v} \mathcal{H}\left(\overline{\mathrm{V}}^{v} \mathrm{C}, d\right)^{S_{v}} .
$$

Cohomology is often easier to calculate on the space $\overline{\mathrm{V}} \mathrm{C}$ with distinguishable vertices.

The more interesting use in this paper will be an intermediate step: distinguishing one vertex and indistinguishing other vertices. On $\overline{\mathrm{V}}^{v} \mathrm{C}$ there is the action of $S_{v-1}$ that permutes first $v-1$ vertices, while leaving the last vertex fixed. It is the sub-action of the action of the whole $S_{v}$. We define

$$
\dot{\mathrm{V}}^{v} \mathrm{C}:=\left(\overline{\mathrm{V}}^{v} \mathrm{C}\right)^{S_{v-1}} .
$$

Let us consider the inclusion $i: \dot{\mathrm{V}}^{v} \mathrm{C} \hookrightarrow \overline{\mathrm{V}}^{v} \mathrm{C}$ and symmetrization map $s: \overline{\mathrm{V}}^{v} \mathrm{C} \hookrightarrow \mathrm{V}^{v} \mathrm{C}$. The following proposition states that it is enough to consider classes of $\mathcal{H}\left(\dot{\mathrm{V}}^{v} \mathrm{C}\right)$ in finding $\mathcal{H}\left(\mathrm{V}^{v} \mathrm{C}\right)$.

Proposition A.2. Let $\left(\overline{\mathrm{V}}^{v} \mathrm{C}, \delta\right)$ be a graph complex with $v$ distinguishable vertices, and let $S_{v}$ act by permuting all vertices and $S_{v-1}$ act by permuting first $v-1$ vertices. Let $\mathrm{V}^{v} \mathrm{C}=\left(\overline{\mathrm{V}}^{v} \mathrm{C}\right)^{S_{v}}$ and $\dot{\mathrm{V}}^{v} \mathrm{C}=\left(\overline{\mathrm{V}}^{v} \mathrm{C}\right)^{S_{v-1}}$. Then

$$
\mathcal{H}\left(\mathrm{V}^{v} \mathrm{C}, d\right)=\operatorname{si}\left(\mathcal{H}\left(\dot{\mathrm{V}}^{v} \mathrm{C}, d\right)\right),
$$

where $i: \dot{\mathrm{V}}^{v} \mathrm{C} \hookrightarrow \overline{\mathrm{V}}^{v} \mathrm{C}$ is inclusion and $s: \overline{\mathrm{V}}^{v} \mathrm{C} \hookrightarrow \mathrm{V}^{v} \mathrm{C}$ is a symmetrization map.

Proof. Standard result. Note that graph complexes are over a field of characteristic zero. 


\section{B Technical results}

\section{B.1 Unbounded remainder}

The following lemma calculates the cohomology of $\left(\mathrm{UR}_{-1, n}, \delta+\Delta\right)$ defined in Definition 4.12

Lemma B.1. $\mathcal{H}\left(\mathrm{UR}_{-1, n}, \delta+\Delta\right)$ is 1-dimensional, the class being represented by $\alpha=\sum_{n \geq 1} \frac{1}{n !} \sigma_{1}^{\cup n}$.

Proof. On UR $-1, n$ we set up a spectral sequence on the number of vertices. The spectral sequence clearly converges correctly. The first differential is $\Delta$. In the first row there is just $\sigma_{1}$, and it survives on the first page. All other rows have two terms, one with and one without $\lambda_{2}$, that cancel on the first page. Therefore, the cohomology must be 1-dimensional. One easily checks that $(\delta+\Delta) \alpha=0$ and since $\alpha$ has $\sigma_{1}$ in the first row, it represents the class.

\section{B.2 Trivial vertex splitting}

The following lemma shows that the differential that splits vertices without cancelling terms "adding an edge" and "extracting a hair", makes cohomology trivial. The result is not the most general one, but adjusted to our needs in Proposition 4.13.

Lemma B.2. The complex $\mathrm{fHGC}_{-1, n}^{\ddagger}$ with the differential $\Gamma \mapsto \Delta(\Gamma)+\sum_{x \in V(\Gamma)} \frac{1}{2} s_{x}$ is acyclic.

Proof. Let an antenna be a maximal connected subgraph consisting of at least one 1-valent vertex and 2-valent vertices. Note that there are two kinds of linear graphs (those that do not have 3- or more-valent vertex) that are entirely considered an antenna: hairless one (that is longer than two vertices) and the one with a hair on one end. They can also be a connected component in a graph, and we call them linear antennas. Moreover, vertices that are not part of an antenna are called quasi-antennas.

The length of an antenna is the number of vertices in it, and the length of a quasi-antenna is zero. Let $l$ be the total length of all antennas in the graph. We set up a spectral sequence on the number $l-e$. The differential can not increase that number. To ensure the correct converging of the spectral sequence, we split the complex into the product of complexes for fixed $c=e+h-v$. For fixed degree $e, h-v$ is fixed too. Since from the definition of fHGC ${ }_{-1, n}^{\ddagger}$ there is no $\sigma_{1}$ in the graph as a connected component, with the fixed number of edges, the number of vertices $v$ is bounded, and so is the number of hairs $h$. Therefore, for the fixed degree $e$ and the row in the spectral sequence $l-e$ the space is finite-dimensional, and the standard spectral sequence argument (e.g. 5, Proposition 19]) implies that the spectral sequence converges correctly.

It is easy to check that the first part of the differential is only extending an antenna. When summed all together, even-length antennas (including quasi-antennas, i.e. vertices that are not in an antenna) are extended by one vertex, and odd-length antennas are sent to 0 . For linear antennas it is the opposite. There is a homotopy that contracts an odd-length antenna and even-length linear antenna different than $\lambda$ (because the result would be $\sigma$ what is not allowed). There is always an antenna (at least quasi-antenna) in the graph, so the homotopy implies the acyclicity.

\section{B.3 Results for Subsection 7.1}

The following three lemmas are necessarily calculations used in the proof of Propositions 7.3 and 7.4

Lemma B.3. $\left(\mathrm{A}^{a} \dot{\mathrm{V}}^{3} \mathrm{fHGC}_{-1,0}, \Delta\right)$ is acyclic for odd $a \geq 3$.

Proof. Like in the proof of Propositions 7.3 and 7.4 we set up a spectral sequence on the total valence of nonchosen vertices, including hairs, and get two classes that survive second page: $\lambda_{1} \dot{\cup} \sigma_{a-1}$ and $\dot{c}\left(\lambda_{a}\right)$. Let

$$
\xi:=\sum_{i=1}^{a-1} \sum_{j=0}^{a-i-1}(-1)^{i}\left(\begin{array}{c}
a-1 \\
i, j, a-i-j-1
\end{array}\right) \bigoplus_{a-i-j-1}^{\substack{i \\
j}} \in \dot{V}_{3}^{a}
$$

where the upper vertex is chosen. We will work also with

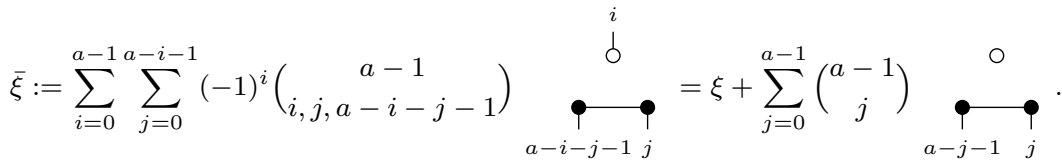


It is not an element of $\mathrm{A}^{a} \dot{\mathrm{V}}^{3} \mathrm{fHGC}_{-1,0}$ because it has terms with an isolated vertex. It holds that

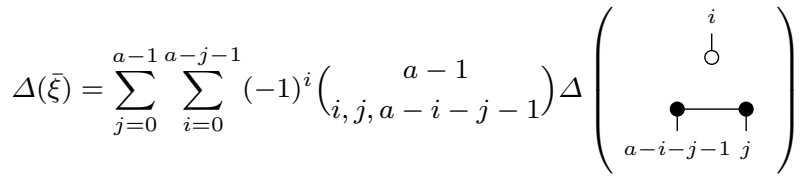

$$
\begin{aligned}
& =2 \sum_{j=0}^{a-1} \sum_{i=1}^{a-j-1}(-1)^{i} \frac{(a-1) !}{(i-1) ! j !(a-i-j-1) !} \\
& +2 \sum_{j=0}^{a-1} \sum_{i=0}^{a-j-2}(-1)^{i} \frac{(a-1) !}{i ! j !(a-i-j-2) !} \bigodot_{a-i-j-2}^{i} j=0 \text {, }
\end{aligned}
$$

The second sum after substitution $i-1 \rightarrow i$ gives exactly negative of the first sum, hence the result is zero. Recall that $\dot{c}(\Gamma)=\Delta_{0}\left(\Gamma \dot{\cup} \sigma_{0}\right)$ where $\dot{\cup} \sigma_{0}$ means adding a stair whose dot becomes chosen, and $\Delta_{0}$ connects a hair to the chosen vertex. Therefore

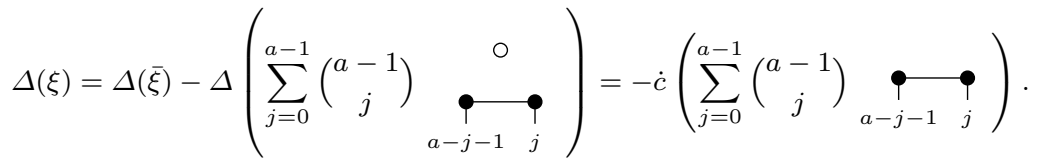

Let

$$
\begin{gathered}
\nu:=\sum_{j=1}^{a-1}\left(\begin{array}{l}
a \\
j
\end{array}\right) \underset{a-j}{\bullet} \underset{j}{\bullet}, \\
\bar{\nu}:=\sum_{j=0}^{a}\left(\begin{array}{l}
a \\
j
\end{array}\right) \underset{a-j}{\bullet} \underset{j}{\bullet}=\nu+2 \underset{a}{\bullet} \bullet .
\end{gathered}
$$

It holds that

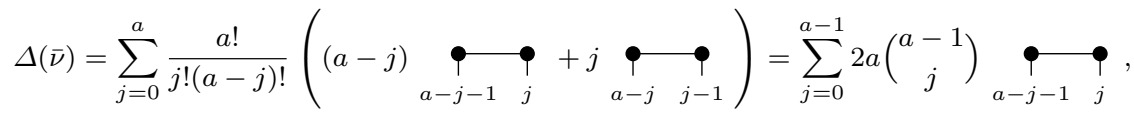

$$
\begin{aligned}
& \Delta(\nu)=\Delta(\bar{\nu})-2 a \lambda_{a}, \\
& \Delta(2 a \xi+\dot{c}(\nu))=2 a \Delta(\xi)+\dot{c}(\Delta(\nu))=-2 a \dot{c}\left(\lambda_{a}\right) .
\end{aligned}
$$

Element $2 a \xi+\dot{c}(\nu)$ contains $\lambda_{1} \dot{\cup} \sigma_{a-1}$ that survives the second page of the spectral sequence, and the target is exactly multiple of $\lambda_{a}$ that also survives, so the two cancel each other. That was to be demonstrated.

Recall 77.2 :

$$
\rho_{a}:=\sum_{i=1}^{a-1} \frac{(-1)^{i}}{i !(a-1-i) !} \sigma_{i} \cup \lambda_{a-i} \in \mathrm{A}^{a} \mathrm{~V}^{3} \mathrm{fHGC}_{-1,0}
$$

Lemma B.4. For even $a \geq 2$ it holds that

$$
\Delta\left(\rho_{a}\right)=0 .
$$


Proof.

$$
\begin{aligned}
\Delta\left(\rho_{a}\right)= & \sum_{i=1}^{a-1} \frac{(-1)^{i}}{i !(a-i-1) !}(\underset{a-i-1}{\bullet}) \\
= & \sum_{i=1}^{a-1} \frac{(-1)^{i}}{(i-1) !(a-i-1) !} \\
& +\sum_{i=1}^{a-1} \frac{(-1)^{i}}{(i-1) !(a-i-1) !} \bullet_{a-i-1}^{i} \\
& +\sum_{i=1}^{a-2} \frac{(-1)^{i}}{i !(a-i-2) !}
\end{aligned}
$$

Addend $i=1$ of the first term is zero because of symmetry reasons, exchanging two edges gives the sign -1 . Other addends of the first term for $i$ cancel the third term for $i-1$, while the second term is easily seen to be 0 for even $a$, concluding the proof.

Lemma B.5. For even $a \geq 2$ it holds that

$$
c\left(\rho_{a}\right)=0 .
$$

Proof. Because of the symmetry $c\left(\lambda_{a}\right)=0$ for every $a \geq 1$. Therefore

$$
c\left(\rho_{a}\right)=\sum_{i=1}^{a-1} \frac{(-1)^{i}}{i !(a-1-i) !} c\left(\sigma_{i}\right) \cup \lambda_{a-i}=\sum_{i=1}^{a-1} \frac{(-1)^{i}}{(i-1) !(a-1-i) !} \lambda_{i} \cup \lambda_{a-i}
$$

Exchanging edges gives $\lambda_{i} \cup \lambda_{a-i}=-\lambda_{a-1} \cup \lambda_{i}$, so the substitution $i \rightarrow a-1$ gives

$$
c\left(\rho_{a}\right)=-\sum_{i=1}^{a-1} \frac{(-1)^{a-1}}{(i-1) !(a-1-i) !} \lambda_{i} \cup \lambda_{a-i}
$$

For $a$ even this is exactly negative of the upper expression, so $c\left(\rho_{a}\right)=0$.

B.4 The morphisms $\pi_{f}$

The following three lemmas are needed to define the morphisms $\pi_{f}$ in Subsection 7.6 Recall 7.9 :

$$
\Sigma_{m}=\sum_{\substack{k_{i} \geq 0 \\ \sum_{i} i k_{i}=m}} \prod_{i>0} \frac{(-1)^{k_{i}}}{k_{i} !((2 i+1) !)^{k_{i}}} \bigcup_{i>0} \sigma_{2 i+1}^{\cup k_{i}}
$$

and that we mean $\Gamma \cup \Sigma_{0}=\Gamma$.

Lemma B.6. For every $m \geq 1$ it holds that

$$
(\delta+\Delta)\left(\Sigma_{m}\right)=0 .
$$


Proof. We first calculate $\delta\left(\Sigma_{m}\right)$. In all sums, products and unions $i, i^{\prime}$ and $j$ are $\geq 1$.

$$
\begin{aligned}
& \delta\left(\Sigma_{m}\right)=\sum_{\substack{k_{i} \geq 0 \\
\sum_{i} i k_{i}=m}} \prod_{i} \frac{(-1)^{k_{i}}}{k_{i} !((2 i+1) !)^{k_{i}}}\left(\sum_{i} k_{i} \delta\left(\sigma_{2 i+1}\right) \cup \sigma_{2 i+1}^{\cup k_{i}-1} \cup \bigcup_{j \neq i} \sigma_{2 j+1}^{\cup k_{j}}\right)= \\
& =\sum_{\substack{k_{i} \geq 0 \\
\sum_{i} i k_{i}=m}} \sum_{\substack{i \\
k_{i}>0}} \frac{(-1)^{k_{i}}}{\left(k_{i}-1\right) !((2 i+1) !)^{k_{i}}} \prod_{j \neq i} \frac{(-1)^{k_{j}}}{k_{j} !((2 j+1) !)^{k_{j}}} \sum_{h=1}^{i-1}\left(\begin{array}{c}
2 i+1 \\
2 h
\end{array}\right) \underset{2 h 2 i-2 h+1}{\bullet} \cup \sigma_{2 i+1}^{\cup k_{i}-1} \cup \bigcup_{j \neq i} \sigma_{2 j+1}^{\cup k_{j}}= \\
& =-\sum_{\substack{k_{i} \geq 0 \\
\sum_{i} i k_{i}=m}} \sum_{\substack{i \\
k_{i}>0}} \sum_{h=1}^{i-1}\left(\frac{1}{(2 h) !(2 i-2 h+1) !} \underset{2 h 2 i-2 h+1}{\bullet} \underset{\bullet}{\bullet}\right) \\
& \cup\left(\frac{1}{\left(k_{i}-1\right) !}\left(\frac{-1}{(2 i+1) !} \sigma_{2 i+1}\right)^{\cup k_{i}-1}\right) \cup \bigcup_{j \neq i}\left(\frac{1}{k_{j} !}\left(\frac{-1}{(2 j+1) !} \sigma_{2 j+1}\right)^{\cup k_{j}}\right)= \\
& =-\sum_{i, i^{\prime}} \sum_{\substack{k_{j} \geq 0 \\
i+i^{\prime}+\sum_{j} j k_{j}=m}}\left(\frac{1}{(2 i) !\left(2 i^{\prime}+1\right) !} \underset{2 i}{\stackrel{\bullet}{\longrightarrow}} \stackrel{\bullet}{\rightarrow i^{\prime}+1}\right) \cup \bigcup_{j}\left(\frac{1}{k_{j} !}\left(\frac{-1}{(2 j+1) !} \sigma_{2 j+1}\right)^{\cup k_{j}}\right) \text {. }
\end{aligned}
$$

Let us now calculate $\Delta\left(\Sigma_{m}\right)$ :

$$
\begin{aligned}
& \Delta\left(\Sigma_{m}\right)=\sum_{\substack{k_{i} \geq 0 \\
\sum_{i} i k_{i}=m}} \prod_{i} \frac{(-1)^{k_{i}}}{k_{i} !((2 i+1) !)^{k_{i}}}\left(\sum_{i} k_{i}\left(k_{i}-1\right)(2 i+1) \underset{2 i}{\bullet} \underset{2 i+1}{\bullet} \cup \sigma_{2 i+1}^{\cup k_{i}-2} \cup \bigcup_{j \neq i} \sigma_{2 j+1}^{\cup k_{j}}+\right. \\
& \left.+\sum_{\substack{i, i^{\prime} \\
i \neq i^{\prime}}} k_{i} k_{i^{\prime}}(2 i+1) \underset{2 i}{\bullet} \underset{2 i^{\prime}+1}{\bullet} \cup \sigma_{2 i+1}^{\cup k_{i}-1} \cup \sigma_{2 i^{\prime}+1}^{\cup k_{i^{\prime}}-1} \cup \bigcup_{j \neq i, i^{\prime}} \sigma_{2 j+1}^{\cup k_{j}}\right)= \\
& =\sum_{\substack{k_{i} \geq 0 \\
\sum_{i} i k_{i}=m}} \sum_{\substack{i \\
k_{i} \geq 2}}\left(\frac{1}{(2 i) !(2 i+1) !} \underset{2 i}{\bullet} \underset{2 i+1}{\bullet}\right) \cup\left(\frac{1}{\left(k_{i}-2\right) !}\left(\frac{-1}{(2 i+1) !} \sigma_{2 i+1}\right)^{\cup k_{i}-2}\right) \cup \\
& \cup \bigcup_{j \neq i}\left(\frac{1}{k_{j} !}\left(\frac{-1}{(2 j+1) !} \sigma_{2 j+1}\right)^{\cup k_{j}}\right)+
\end{aligned}
$$

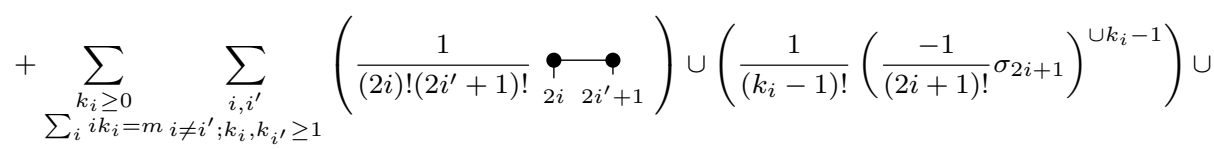

$$
\begin{aligned}
& \cup\left(\frac{1}{\left(k_{i^{\prime}}-1\right) !}\left(\frac{-1}{\left(2 i^{\prime}+1\right) !} \sigma_{2 i^{\prime}+1}\right)^{\cup k_{i^{\prime}}-1}\right) \cup \bigcup_{j \neq i}\left(\frac{1}{k_{j} !}\left(\frac{-1}{(2 j+1) !} \sigma_{2 j+1}\right)^{\cup k_{j}}\right)= \\
& =\sum_{i} \sum_{\substack{k_{j} \geq 0 \\
2 i+\sum_{j}^{j} j k_{j}=m}}\left(\frac{1}{(2 i) !(2 i+1) !} \underset{2 i}{\bullet} \underset{2 i+1}{\bullet}\right) \cup \bigcup_{j}\left(\frac{1}{k_{j} !}\left(\frac{-1}{(2 j+1) !} \sigma_{2 j+1}\right)^{\cup k_{j}}\right) \\
& +\sum_{\substack{i, i^{\prime} \\
i \neq i^{\prime}}} \sum_{\substack{k_{j} \geq 0 \\
i+i^{\prime}+\sum_{j} j k_{j}=m}}\left(\frac{1}{(2 i) !\left(2 i^{\prime}+1\right) !} \underset{2 i}{\bullet} \underset{2 i^{\prime}+1}{\bullet}\right) \cup \bigcup_{j}\left(\frac{1}{k_{j} !}\left(\frac{-1}{(2 j+1) !} \sigma_{2 j+1}\right)^{\cup k_{j}}\right) \\
& =\sum_{i, i^{\prime}} \sum_{\substack{k_{j} \geq 0 \\
i+i^{\prime}+\sum_{j} j k_{j}=m}}\left(\frac{1}{(2 i) !\left(2 i^{\prime}+1\right) !} \underset{2 i}{\stackrel{\bullet}{\bullet}} \underset{2 i^{\prime}+1}{\bullet}\right) \cup \bigcup_{j}\left(\frac{1}{k_{j} !}\left(\frac{-1}{(2 j+1) !} \sigma_{2 j+1}\right)^{\cup k_{j}}\right) .
\end{aligned}
$$

We get exactly the negative expression of $\delta\left(\Sigma_{m}\right)$, so $(\delta+\Delta)\left(\Sigma_{m}\right)=0$. 
Lemma B.7. For every $\Gamma \in \mathrm{fGC}_{0}^{\geq 1}$ and $m \geq 0$ it holds that

$$
\begin{gathered}
\begin{array}{c}
\sum_{n=0}^{m-1} \frac{1}{(2 n) !}\left(\Delta\left(\chi^{2 n}(\Gamma) \cup \Sigma_{m-n}\right)-\Delta \chi^{2 n}(\Gamma) \cup \Sigma_{m-n}-\chi^{2 n}(\Gamma) \cup \Delta\left(\Sigma_{m-n}\right)\right) \\
=-\sum_{n=0}^{m} \frac{1}{(2 n) !}\left(\delta \chi^{2 n}(\Gamma)-\chi^{2 n} \delta(\Gamma)\right) \cup \Sigma_{m-n} \\
\sum_{n=1}^{m-1} \frac{1}{(2 n-1) !}\left(\Delta\left(\chi^{2 n-1}(\Gamma) \cup \Sigma_{m-n}\right)-\Delta \chi^{2 n-1}(\Gamma) \cup \Sigma_{m-n}-\chi^{2 n-1}(\Gamma) \cup \Delta\left(\Sigma_{m-n}\right)\right)= \\
=-\sum_{n=1}^{m} \frac{1}{(2 n-1) !}\left(\delta \chi^{2 n-1}(\Gamma)-\chi^{2 n-1} \delta(\Gamma)\right) \cup \Sigma_{m-n}
\end{array}
\end{gathered}
$$

Proof. Let us prove only the first equation, while the second equation is similar. We make notations such that the claim is $\sum L_{n}=-\sum R_{n} . L_{n}$ is exactly the part of $\Delta\left(\chi^{2 n}(\Gamma) \cup \Sigma_{m-n}\right)$ that connects $\chi^{2 n}(\Gamma)$ with $\Sigma_{m-n}$. It can be done in two ways, a hair from $\chi^{2 n}(\Gamma)$ connects to a star in $\Sigma_{m-n}$, or a star from $\Sigma_{m-n}$ connects to $\chi^{2 n}(\Gamma)$. In both cases "a flower" with 2 or more hairs is added to $\chi^{2 n}(\Gamma)$. Let $f_{k}$ be a map that adds a flower with $k$ hairs to a vertex in all possible ways. In all sums $i$ and $j$ are $\geq 1$. It holds that:

$$
\begin{aligned}
& L_{n}=\frac{1}{(2 n) !} \sum_{\substack{k_{j} \geq 0 \\
\sum_{j} j k_{j}=m-n}} \prod_{j} \frac{(-1)^{k_{j}}}{k_{j} !((2 j+1) !)^{k_{j}}} \sum_{i} k_{i}\left(2 n f_{2 i+1} \chi^{2 n-1}(\Gamma)+(2 i+1) f_{2 i} \chi^{2 n}(\Gamma)\right) \cup \sigma_{2 i+1}^{\cup k_{i}-1} \cup \bigcup_{j \neq i} \sigma_{2 j+1}^{\cup k_{j}}= \\
& =-\sum_{i} \sum_{\substack{k_{j} \geq 0 \\
i+\sum_{j} j k_{j}=m-n}}\left(\frac{1}{(2 i+1) !(2 n-1) !} f_{2 i+1} \chi^{2 n-1}(\Gamma)+\frac{1}{(2 i) !(2 n) !} f_{2 i} \chi^{2 n}(\Gamma)\right) \cup \bigcup_{j}\left(\frac{1}{k_{j} !}\left(\frac{-1}{(2 j+1) !} \sigma_{2 j+1}\right)^{\cup k_{j}}\right)= \\
& =-\sum_{i}\left(\frac{1}{(2 i+1) !(2 n-1) !} f_{2 i+1} \chi^{2 n-1}(\Gamma)+\frac{1}{(2 i) !(2 n) !} f_{2 i} \chi^{2 n}(\Gamma)\right) \cup \Sigma_{m-n-i} .
\end{aligned}
$$

On the other side there is

$$
R_{n}=\frac{\Sigma_{m-n}}{(2 n) !} \cup \sum_{x}\left(\frac{1}{2} s_{x}\left(\chi^{2 n}(\Gamma)\right)-a_{x}\left(\chi^{2 n}(\Gamma)\right)-h(x) e_{x}\left(\chi^{2 n}(\Gamma)\right)-\frac{1}{2} \chi^{2 n}\left(s_{x}(\Gamma)\right)+\chi^{2 n}\left(a_{x}(\Gamma)\right)\right),
$$

where $x$ runs through vertices of $\Gamma$. It is clear that $s_{x}\left(\chi^{2 n}(\Gamma)\right)=\chi^{2 n}\left(s_{x}(\Gamma)\right)$. In $\chi^{2 n}\left(a_{x}(\Gamma)\right)$ hairs are added to vertices of $\Gamma$ or to the new vertex of the antenna. Terms $-a_{x}\left(\chi^{2 n}(\Gamma)\right)$ and $-h(x) e_{x}\left(\chi^{2 n}(\Gamma)\right)$ cancel exactly the terms of $\chi^{2 n}\left(a_{x}(\Gamma)\right)$ where no or one hair is added to the new vertex. So

$$
R_{n}=\frac{\Sigma_{m-n}}{(2 n) !} \cup \sum_{i=2}^{2 n}\left(\begin{array}{c}
2 n \\
i
\end{array}\right) f_{i}\left(\chi^{2 n-i}(\Gamma)\right)=\sum_{i=2}^{2 n} \frac{1}{i !(2 n-i) !} f_{i}\left(\chi^{2 n-i}(\Gamma)\right) \cup \Sigma_{m-n} .
$$

Now a simple play with the sums leads to the result.

Lemma B.8. For every $\Gamma \in \mathrm{B}^{b} \mathrm{fGC}_{0}^{\geq 1}$ and $m \in \mathbb{Z}$ of the same parity as $b$ it holds that

$$
(\delta+\Delta) \pi_{m}(\Gamma)=\pi_{m}(\delta+D \nabla)(\Gamma)
$$

in the space $\mathrm{fHGC}_{-1,0}^{\ddagger}$.

Proof. The left-hand side is

$$
\begin{aligned}
(\delta+\Delta) \pi_{m}(\Gamma)= & \delta+\Delta)\left(\sum_{n=0}^{\frac{m-b}{2}} \frac{1}{(2 n) !} \chi^{2 n}(\Gamma) \cup \Sigma_{\frac{m-b}{2}-n}-\sum_{n=1}^{\frac{m-b}{2}} \frac{1}{(2 n-1) !} \chi^{2 n-1} D(\Gamma) \cup \Sigma_{\frac{m-b}{2}-n}\right)= \\
= & \sum_{n=0}^{\frac{m-b}{2}} \frac{\delta\left(\chi^{2 n}(\Gamma)\right)}{(2 n) !} \cup \Sigma_{\frac{m-b}{2}-n}+\sum_{n=0}^{\frac{m-b}{2}-1} \frac{\chi^{2 n}(\Gamma)}{(2 n) !} \cup \delta \Sigma_{\frac{m-b}{2}-n} \\
& -\sum_{n=1}^{\frac{m-b}{2}} \frac{\delta\left(\chi^{2 n-1} D(\Gamma)\right)}{(2 n-1) !} \cup \Sigma_{\frac{m-b}{2}-n}+\sum_{n=1}^{\frac{m-b}{2}-1} \frac{\chi^{2 n-1} D(\Gamma)}{(2 n-1) !} \cup \delta \Sigma_{\frac{m-b}{2}-n} \\
& +\sum_{n=0}^{\frac{m-b}{2}} \frac{\Delta\left(\chi^{2 n}(\Gamma) \cup \Sigma_{\frac{m-b}{2}-n}\right)}{(2 n) !}-\sum_{n=1}^{\frac{m-b}{2}} \frac{\Delta\left(\chi^{2 n-1} D(\Gamma) \cup \Sigma_{\frac{m-b}{2}-n}\right)}{(2 n-1) !} .
\end{aligned}
$$


Using Lemmas 2.5 B.6 and B.7 and Proposition 3.3 it follows that:

$$
\begin{aligned}
(\delta+\Delta) \pi_{m}(\Gamma)= & \sum_{n=0}^{\frac{m-b}{2}} \frac{\chi^{2 n} \delta(\Gamma)}{(2 n) !} \cup \Sigma_{\frac{m-b}{2}-n}+\sum_{n=0}^{\frac{m-b}{2}-1} \frac{\chi^{2 n}(\Gamma)}{(2 n) !} \cup \delta \Sigma_{\frac{m-b}{2}-n} \\
& -\sum_{n=1}^{\frac{m-b}{2}} \frac{\chi^{2 n-1} \delta D(\Gamma)}{(2 n-1) !} \cup \Sigma_{\frac{m-b}{2}-n}+\sum_{n=1}^{\frac{m-b}{2}-1} \frac{\chi^{2 n-1} D(\Gamma)}{(2 n-1) !} \cup \delta \Sigma_{\frac{m-b}{2}-n} \\
& +\sum_{n=0}^{\frac{m-b}{2}} \frac{\Delta \chi^{2 n}(\Gamma) \cup \Sigma_{\frac{m-b}{2}}-n}{(2 n) !}+\sum_{n=0}^{\frac{m-b}{2}-1} \frac{\chi^{2 n}(\Gamma) \cup \Delta \Sigma_{\frac{m-b}{2}-n}}{(2 n) !} \\
& -\sum_{n=1}^{\frac{m-b}{2}} \frac{\Delta \chi^{2 n-1} D(\Gamma) \cup \Sigma_{\frac{m-b}{2}}-n}{(2 n-1) !}+\sum_{n=1}^{\frac{m-b}{2}-1} \frac{\chi^{2 n-1} D(\Gamma) \cup \Delta \Sigma_{\frac{m-b}{2}-n}}{(2 n-1) !}= \\
= & \sum_{n=0}^{\frac{m-b}{2}}\left(\frac{\chi^{2 n} \delta(\Gamma)}{(2 n) !} \cup \Sigma_{\frac{m-b}{2}-n}\right)-\sum_{n=1}^{\frac{m-b}{2}}\left(\frac{\chi^{2 n-1} D \delta(\Gamma)}{(2 n-1) !} \cup \Sigma_{\frac{m-b}{2}}\right) \\
& +\sum_{n=1}^{\frac{m-b}{2}} \frac{\chi^{2 n-2} D \nabla(\Gamma)}{(2 n-2) !} \cup \Sigma_{\frac{m-b}{2}-n} .
\end{aligned}
$$

The right-hand side of the claim is $\pi_{m}(\delta(\Gamma)+D \nabla(\Gamma))$. The first term under $\pi_{m}$ is in $\mathrm{B}^{b} \mathrm{fGC}_{0}^{\geq 1}$, and the second one is in $\mathrm{B}^{b+2} \mathrm{fGC}_{0}^{\geq 1}$, so by Propositions 3.3 and 3.4 it follows that

$$
\begin{aligned}
\pi_{m}(\delta+D \nabla)(\Gamma)=\sum_{n=0}^{\frac{m-b}{2}} & \frac{1}{(2 n) !} \chi^{2 n} \delta(\Gamma) \cup \Sigma_{\frac{m-b}{2}-n}-\sum_{n=1}^{\frac{m-b}{2}} \frac{1}{(2 n-1) !} \chi^{2 n-1} D \delta(\Gamma) \cup \Sigma_{\frac{m-b}{2}-n} \\
& +\sum_{n=0}^{\frac{m-b}{2}-1} \frac{1}{(2 n) !} \chi^{2 n} D \nabla(\Gamma) \cup \Sigma_{\frac{m-b}{2}-n-1}-\sum_{n=1}^{\frac{m-b}{2}-1} \frac{1}{(2 n-1) !} \chi^{2 n-1} D D \nabla(\Gamma) \cup \Sigma_{\frac{m-b}{2}-n-1} .
\end{aligned}
$$

This is the same result as on the left-hand side, what was to be shown.

\section{New waterfall mechanism}

In this section of appendix we will use other results from the literature and give an idea what these results, together with our result, imply. More precisely, we will explain the "waterfall mechanism" introduced in [6], and give some examples.

There is a Lie bracket on $\mathrm{HGC}_{m, n}$ defined as

$$
\left[\Gamma_{1}, \Gamma_{2}\right]=\Delta\left(\Gamma_{1} \cup \Gamma_{2}\right)-\Delta \Gamma_{1} \cup \Gamma_{2}-\Gamma_{1} \cup \Delta \Gamma_{2},
$$

i.e. it transforms a hair from one graph to an edge connecting to the other graph. Let

$$
h_{1}:=\sum_{k \geq 1} \frac{1}{(2 k+1) !} \sigma_{2 k+1}
$$

It is a Maurer-Cartan element in $\mathrm{HGC}_{n-1, n}$. Recall that $\chi^{(1)}$ adds a hair in all possible ways. We have the following theorem.

Theorem C.1 ( [16, 17 19, 6]). There are quasi-isomorphisms

$$
\begin{aligned}
\left(\mathrm{fGCc}_{n}^{\geq 2}, \delta\right) & \rightarrow\left(\operatorname{HGC}_{n, n}, \delta+\chi^{(1)}\right), \\
\mathbb{K} \oplus\left(\mathrm{fGCc}_{n}^{\geq 2}, \delta\right) & \rightarrow\left(\operatorname{HGC}_{n-1, n}, \delta+\left[h_{1}, \cdot\right]\right) .
\end{aligned}
$$

Furthermore the spectral sequence obtained by the filtration by number of hairs abuts at the second page in the first case.

We call the spectral sequence arising from the deformed differential of Theorem 1.1 the first spectral sequence, and the one arising from the deformed differential of Theorem C.1 the second.

The convergence of both spectral sequences implies that the hairy graph cohomology classes must come "in pairs", apart from classes that come from $\mathrm{fGCc}_{\bar{n}}^{\geq 2}$ in the second spectral sequence. More concretely, given a hairy graph cohomology class $\Gamma$, it will survive up to some page of the spectral sequence, on which it is either killed by or kills (the image of) another hairy graph cohomology class.

Non-loop classes $[\Gamma] \in \mathcal{H}\left(\mathrm{GC}_{n}, \delta\right)$ are sent to $\left[\chi^{(1)}(\Gamma)\right]$ in Theorem C.1 so those classes always have 1 hair. Loop classes are sent differently, see [16] for more details. Let $\Gamma$ live in tri-degree

(cohom. degree, number of hairs, $e-v)=(d, h, b)$. 
Then the "partner class" from the first spectral sequence lives in tri-degree $(d+1, h-j, b+j)($ or $(d-1, h+j, b-j))$ for some yet unknown positive integer $j$. Then the "partner class" from the second spectral sequence lives in tri-degree $(d+1, h+1, b)$ (or $(d-1, h-1, b))$, since the spectral sequence abuts on the second page.

Now, using the constraints provided by the first and the second spectral sequences together, we may construct a large set of hairy graph cohomology classes from (assumed to be known) non-hairy classes. Concretely, we pick up a non-hairy class and embed it into the hairy complex, find its partner from the first spectral sequence, then partner's partner from the second spectral sequence, then its partner again from the first spectral sequence etc., until at some point we reach another hairy graph cohomology class that comes from non-hairy one.

For an illustration of the process, see the computer generated table of the hairy graph cohomology in Figures 19 and 21 in which (some of) the cancellations in the two spectral sequences have been inscribed. In Figure 20 we see a string of 4 classes formed by waterfall mechanism.

This mechanism was described and proven in [6] for even $n$. It was also described for odd $m$ where it was only conjectured.

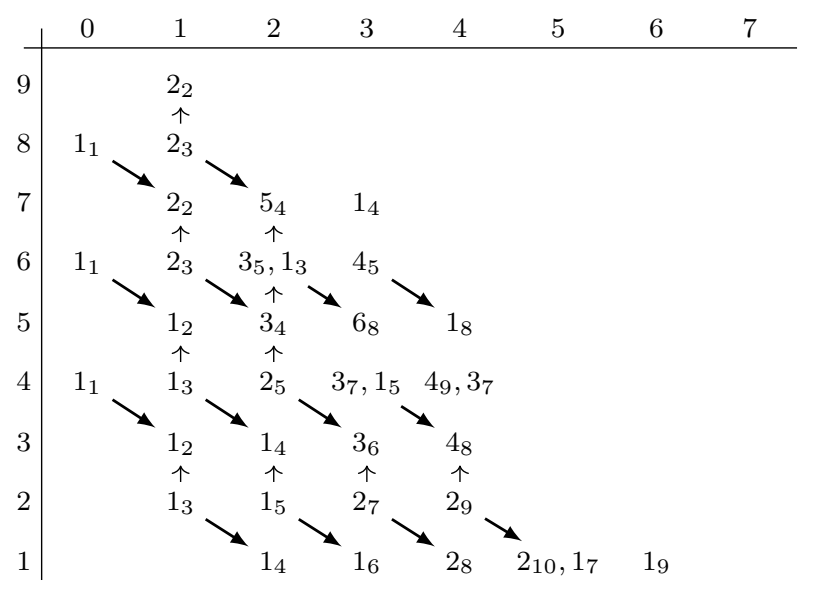

Fig. 19 6, Figure 2] Computer generated table of the dimensions of the hairy graph $\operatorname{cohomology} \operatorname{dim} \mathcal{H}\left(\mathrm{HGC}_{-1,1}\right)$. The rows indicate the number of hairs $h(\uparrow)$, the columns $b=e-v(\rightarrow)$. A table entry $1_{3}$ means that there the degree 3 subspace is one-dimensional. The arrows indicate (some of) the cancellations of classes in the two spectral sequences. The computer program used approximate (floating point) arithmetic, so the displayed numbers should not be considered as rigorous results.

\section{References}

1. Gregory Arone and Victor Turchin. Graph-complexes computing the rational homotopy of high dimensional analogues of spaces of long knots. Ann. Inst. Fourier 65(1), 1-62 (2015).

2. Jim Conant, Jean Costello, Victor Turchin and Patrick Weed. Two-loop part of the rational homotopy of spaces of long embeddings. J. Knot Theory Ramifications 23 (2014), no. 4, 1450018, 23 pp.

3. Marc Culler and Karen Vogtmann. Moduli of graphs and automorphisms of free groups. Invent. Math., 84(1):91-119, 1986.

4. Benoit Fresse, Victor Turchin and Thomas Willwacher. The rational homotopy of mapping spaces of $E_{n}$ operads. arXiv:1703.06123, 2017.

5. Anton Khoroshkin, Thomas Willwacher and Marko Živković. Differentials on graph complexes. Adv. Math. 307:1184$1214,2017$.

6. Anton Khoroshkin, Thomas Willwacher and Marko Živković. Differentials on graph complexes II: Hairy graphs. Lett. Math. Phys. 107: 1781-1797, 2017.

7. Jan Kneissler. The number of primitive Vassiliev invariants up to degree 12. arXiv:q-alg/9706022

8. Maxim Kontsevich. Formal (non)commutative symplectic geometry. In Proceedings of the I. M. Gelfand seminar 1990-1992, 173-188. Birkhauser, 1993

9. Maxim Kontsevich. Formality Conjecture. Deformation Theory and Symplectic Geometry 139-156, 1997. D. Sternheimer et al. (eds.).

10. Sergei Merkulov and Thomas Willwacher. Deformation theory of Lie bialgebra properads. arXiv:1512.05252.

11. Sergei Merkulov and Thomas Willwacher. Props of ribbon graphs, involutive Lie bialgebras and moduli spaces of curves. arXiv:1511.07808.

12. Robert C. Penner. The decorated Teichmüller space of punctured surfaces. Comm. Math. Phys., 113(2):299-339, 1987. 


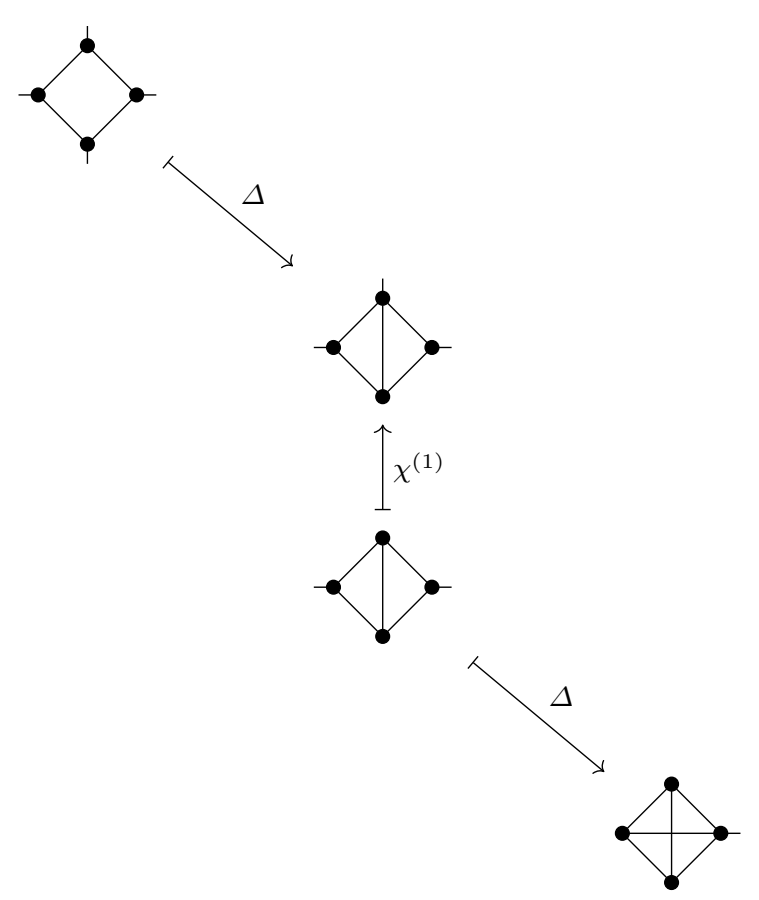

Fig. 20 An example of classes in $\mathrm{HGC}_{-1,1}$ that are cancelled by waterfall mechanism. They represent classes from the leftmost string in Figure 19 The class at the bottom comes from a non-hairy class by adding a hair. The class at the top comes from a non-hairy loop class. We skip graphs with multiple edges because they do not contribute to cohomology.

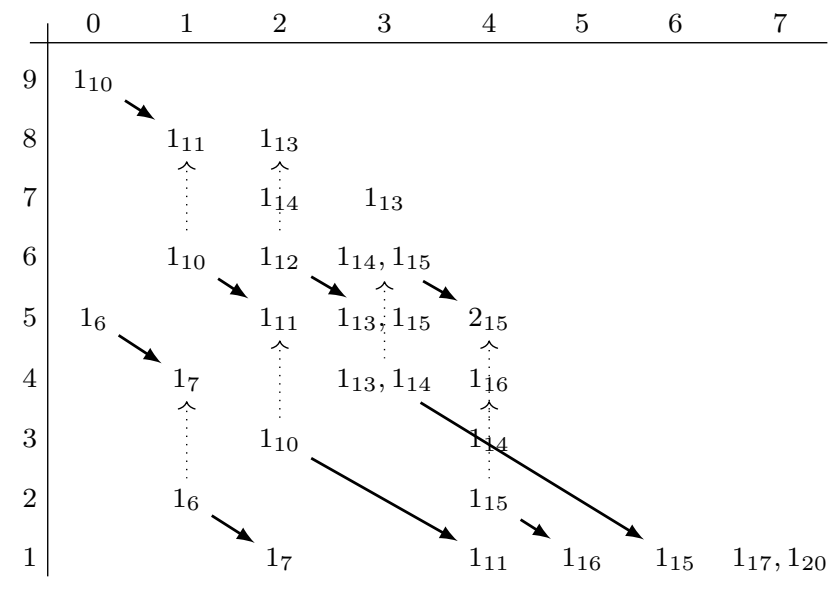

Fig. 21 6, Figure 2] Computer generated table of the dimensions of the hairy graph cohomology $\operatorname{dim} \mathcal{H}\left(\mathrm{HGC}_{-1,0}\right)$. The rows indicate the number of hairs $h(\uparrow)$, the columns $b=e-v(\rightarrow)$. A table entry $1_{3}$ means that there the degree 3 subspace is one-dimensional. The arrows indicate (some of) the cancellations of classes in the two spectral sequences. The computer program used approximate (floating point) arithmetic, so the displayed numbers should not be considered as rigorous results.

13. Robert C. Penner. Perturbative series and the moduli space of Riemann surfaces. Journal of Differential Geometry, 27(1):35-53, 1988.

14. Paul Arnaud Songhafouo Tsopméné and Victor Turchin. Euler characteristics for the Hodge splitting in the rational homology and homotopy of high dimensional string links. arXiv:1609.00778 (2016).

15. Victor Turchin. Hodge-type decomposition in the homology of long knots. J. Topol., 3(3):487-534, 2010.

16. Victor Turchin and Thomas Willwacher. Relative (non-)formality of the little cubes operads and the algebraic Cerf Lemma. arXiv:1409.0163, 2014.

17. Victor Turchin and Thomas Willwacher. Commutative hairy graphs and representations of Out $\left(F_{r}\right)$. arxiv:1603.08855, to appear in J. Top. 
18. Pierre Vogel. Algebraic structures on modules of diagrams. J. Pure Appl. Algebra, 215(6): 1292-1339, 2011.

19. Thomas Willwacher. M. Kontsevich's graph complex and the Grothendieck-Teichmüller Lie algebra. Invent. Math., 200(3):671-760, 2015.

20. Thomas Willwacher, Stable cohomology of polyvector fields Math. Res. Lett., 21 (2014), no. 6, 1501-1530.

21. Thomas Willwacher. The oriented graph complexes. Commun. Math. Phys. 334: 1649, 2015.

22. Thomas Willwacher and Marko Živković. Multiple edges in M. Kontsevich's graph complexes and computations of the dimensions and Euler characteristics. Adv. Math., 272:553-578, 2015.

23. Marko Živković. Multi-directed graph complexes and quasi-isomorphisms between them I: oriented graphs arXiv:1703.09605 